Florida International University FIU Digital Commons

$10-27-2011$

\title{
Measurments of Carotenoid Levels in Human Serum and a Catalog of the Lutein Conformation Populations from Semi-empirical Calculations
}

Vanesa Mendez

Florida International University, vmend008@fiu.edu

DOI: $10.25148 /$ etd.FI11120808

Follow this and additional works at: https://digitalcommons.fiu.edu/etd

\section{Recommended Citation}

Mendez, Vanesa, "Measurments of Carotenoid Levels in Human Serum and a Catalog of the Lutein Conformation Populations from Semi-empirical Calculations" (2011). FIU Electronic Theses and Dissertations. 523.

https://digitalcommons.fiu.edu/etd/523 


\title{
FLORIDA INTERNATIONAL UNIVERSITY
}

Miami, Florida

MEASUREMENT OF CAROTENOID LEVELS IN HUMAN SERUM AND A CATALOG OF THE LUTEIN CONFORMATION POPULATIONS FROM SEMI-EMPIRICAL

\section{CALCULATIONS}

\author{
A thesis submitted in partial fulfillment of the \\ requirements for the degree of \\ MASTER OF SCIENCE \\ in \\ CHEMISTRY \\ by
}

VANESA MENDEZ 
To: Dean Kenneth G. Furton

College of Arts and Sciences

This thesis, written by Vanesa Mendez, and entitled Measurments of Carotenoid Levels in Human Serum and a Catalog of the Lutein Conformation Populations from Semi-empirical Calculations, having been approved in respect to style and intellectual content, is referred to you for judgment.

We have read this thesis and recommend that it be approved.

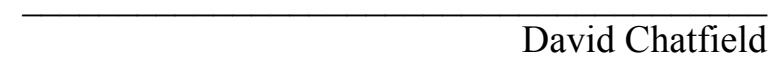

Konstantinos Kavallieratos

John T. Landrum, Major Professor

Date of Defense: October 27, 2011

The thesis of Vanesa Mendez is approved.

Dean Kenneth G. Furton College of Arts and Sciences

Dean Lakshmi N. Reddi University Graduate School

Florida International University, 2011 


\section{DEDICATION}

A mis padres. 


\section{ACKNOWLEDGMENTS}

I wish to express my gratitude to my advisor Dr. John Landrum for having confidence in me and for all the time that he invested teaching and helping me. During these years his guidance, support, and patience have been invaluable for me. I am glad and grateful to have been part of his research group. I would like to thank the members of my committee Dr. Konstantino Kavallieratos, and Dr. David Chatfield for their support and time. I thank Dr. Chatfield, for his help and guidance in one of the projects; Dr. Alexander Mebel, for his help and assistance in some topics of my thesis. Special thanks to Dr. Richard Bone, who was always kindly available to help me and answer any of the questions I had about our projects.

I would like to thank my lab mates for their help and also all the friends that I found in FIU during the past years. It was nice to have their support and companionship during some crazy times. Special thanks to Amanda, Reaskmey, Hasith, and Valerie.

Finally, I would like to express my endless gratitude to my family, especially to my parents for setting an example in my life. They have always believed in me, supported me and cheered for me in all the decisions that I had to make. 


\begin{abstract}
OF THE THESIS
MEASUREMENT OF CAROTENOID LEVELS IN HUMAN SERUM AND A CATALOG OF
\end{abstract}

THE LUTEIN CONFORMATION POPULATIONS FROM SEMI-EMPIRICAL

CALCULATIONS

by

Vanesa Mendez

Florida International University, 2011

Miami, Florida

Professor John T. Landrum, Major Professor

Lutein is a principal constituent of the human macular pigment. This study is composed of two projects. The first studies the conformational geometries of lutein and its potential adaptability in biological systems. The second is a study of the response of human subjects to lutein supplements.

Using semi-empirical parametric method 3 (PM3) and density functional theory with the B3LYP/6-31G* basis set, the relative energies of s-cis conformers of lutein were determined. All 512 s-cis conformers were calculated with PM3. A smaller, representative group was also studied using density functional theory. PM3 results were correlated systematically to B3LYP values and this enables the results to be calibrated. The relative energies of the conformers range from 1-30 $\mathrm{kcal} / \mathrm{mole}$, and many are dynamically accessible at normal temperatures.

Four commercial formulations containing lutein were studied. The serum and macular pigment (MP) responses of human subjects to these lutein supplements with doses of 9 or 20 $\mathrm{mg}$ /day were measured, relative to a placebo, over a six month period. In each instance, lutein levels in serum increased and correlated with MP increases. The results demonstrate that responses are significantly dependent upon formulation and that components other than lutein have an important influence serum response. 


\section{TABLE OF CONTENTS}

CHAPTER

PAGE

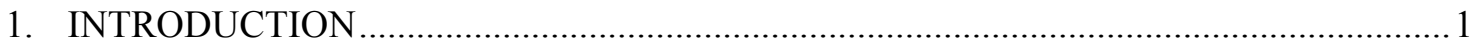

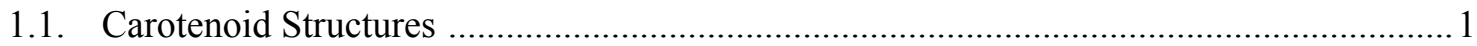

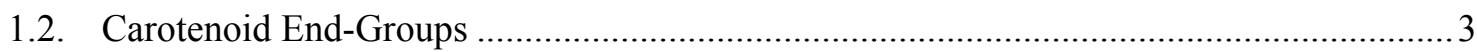

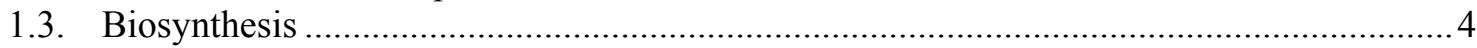

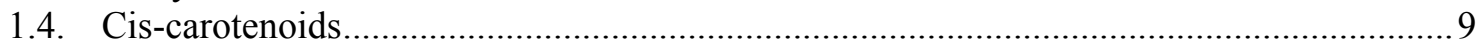

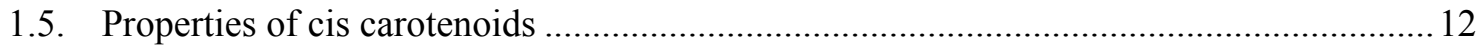

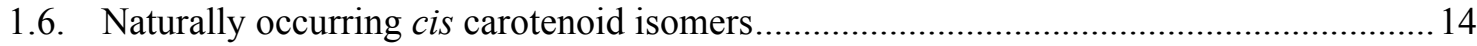

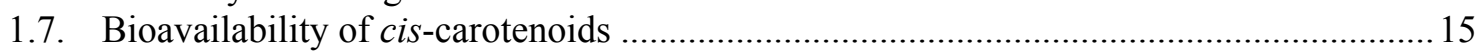

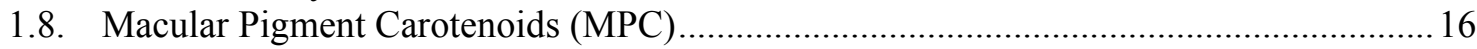

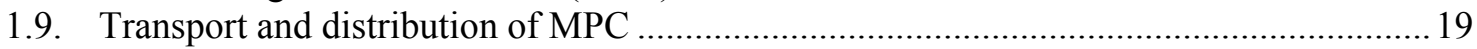

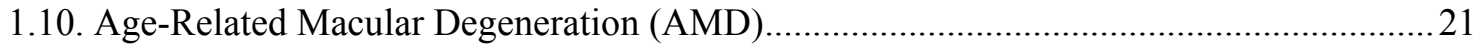

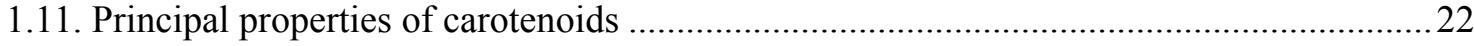

2. A CATALOG OF THE RELATIVE ENERGIES OF LUTEIN CONFORMATION

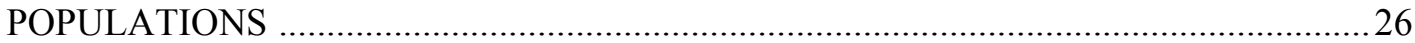

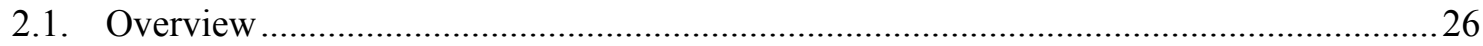

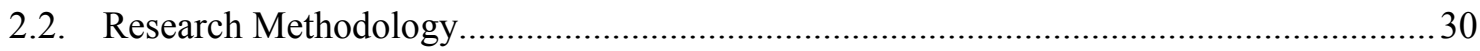

2.2.1. Geometry optimization using the semi-empirical method: PM3 …....................... 30

2.2.2. Geometry optimization using the density functional theory: $B 3 L Y P / 6-31 G^{*} \ldots \ldots . . .31$

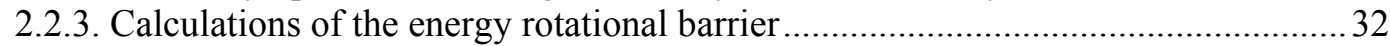

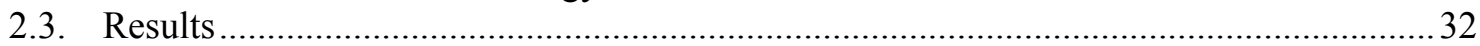

2.3.1. Calculations for the basic conjugated system …............................................... 32

2.3.2. Calculations for the complete carotenoid model using PM3 ............................... 33

2.3.3. Calculations for the complete carotenoid model using B3*LYP/6-31G* .............. 47

2.3.4. Calculations for the Calculation of the rotational barrier of the single bonds in

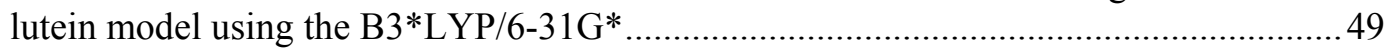

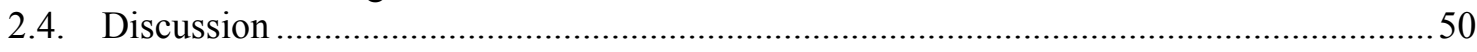

3. MEASUREMENT OF CAROTENOID LEVELS IN HUMAN SERUM.........................56

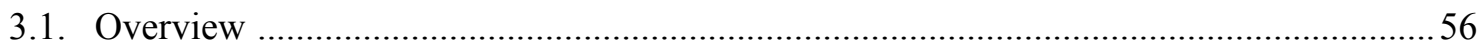

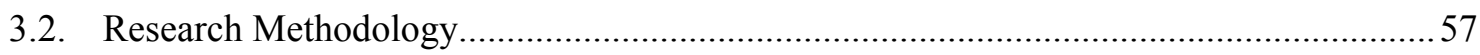

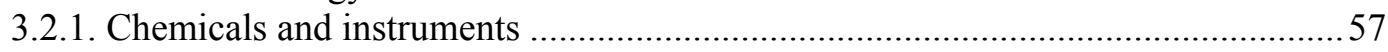

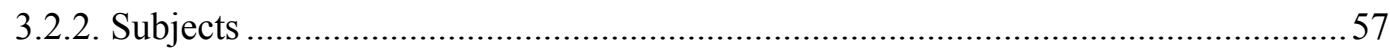

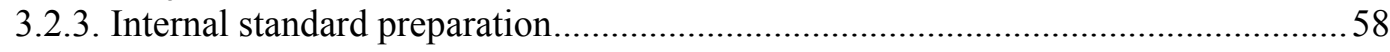

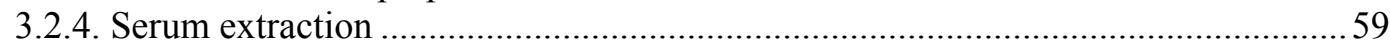

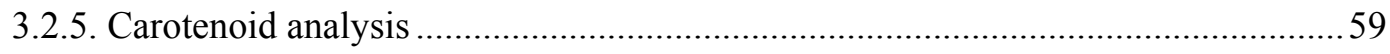

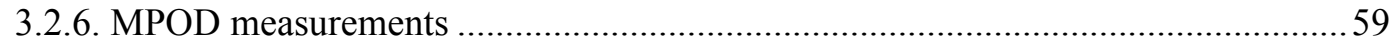

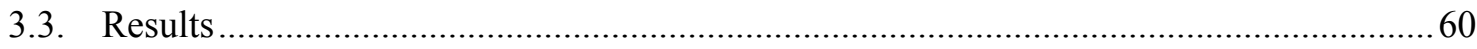

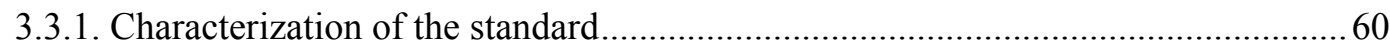

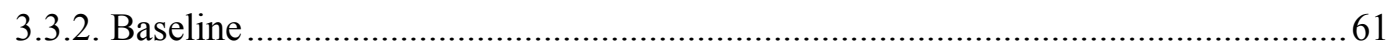

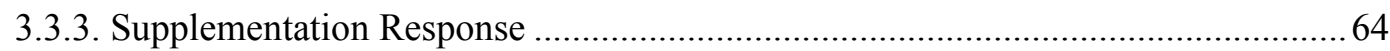

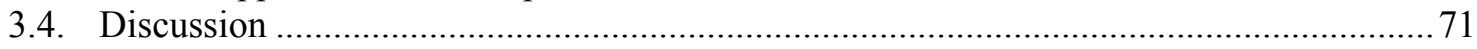

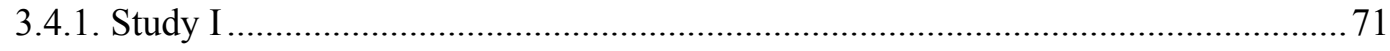

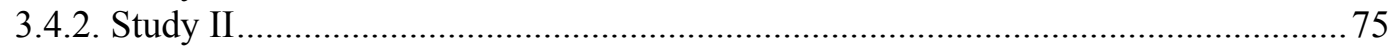

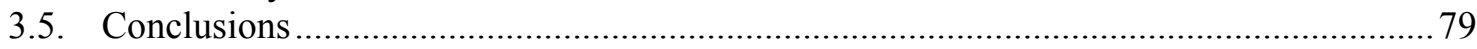


BIBLIOGRAPHY 


\section{LIST OF FIGURES}

FIGURE

PAGE

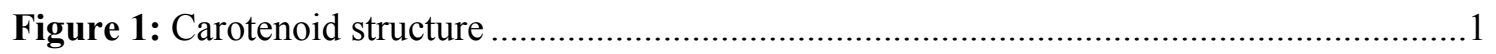

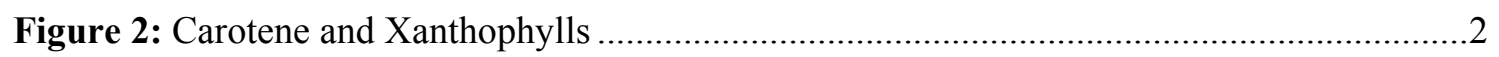

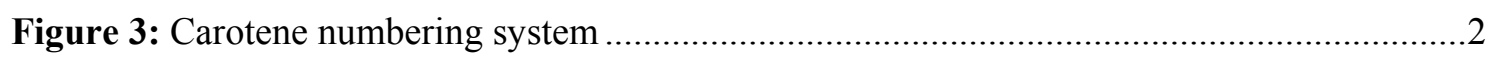

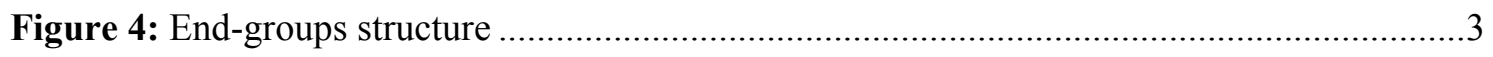

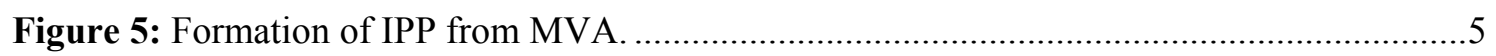

Figure 6: General pathway for the formation of phytoene. The arrows on the side represent the enzymes involved. GGPS: geranylgeranyl diphosphate synthase.

PSY:phytoene synthase.

Figure 7: General pathway for the formation of lycopene. The arrows on the side represent the enzymes involved. PDS: phytoene desaturase. ZDS: $\zeta$-carotene desaturase.

Figure 8: Cyclization of lycopene. Enzymatic conversions are shown by arrows with the enzymes responsible. LCY- $\beta$ : lycopene beta-cyclase; LCY- $\varepsilon$, lycopene epsilon-cyclase; CRH- $\beta, \beta$-carotene hydroxylase; CRH- $\varepsilon$, epsilon-carotene hydroxylase................. 5

Figure 9: Basic mechanism for biosynthesis of $\varepsilon$ and $\beta$-rings in carotenoids...........................

Figure 10.a: Examples of symmetrical carotenoids: violaxanthin,......................................10

Figure 10.b: Examples of symmetrical carotenoids: rhodoxanthin.....................................10

Figure 11.a Representation of a cis double bond in the polyene chain..................................11

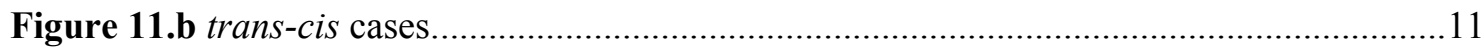

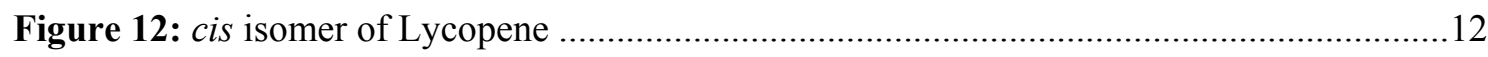

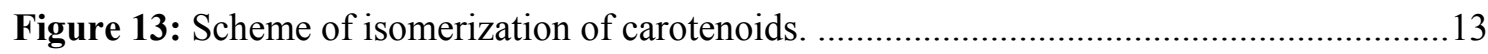

Figure 14: Molar extinction curves of $\beta$-carotene in hexane: all-trans solution, mixture of cistrans isomers after refluxing, mixture after iodine treatment. ................................................ 14

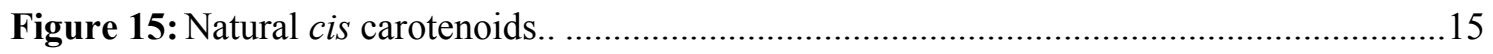

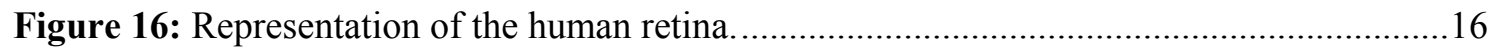

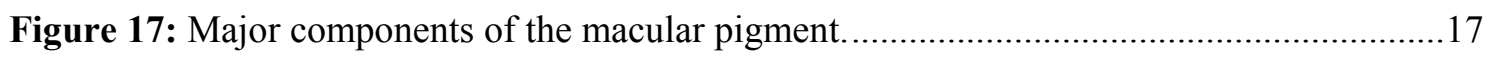

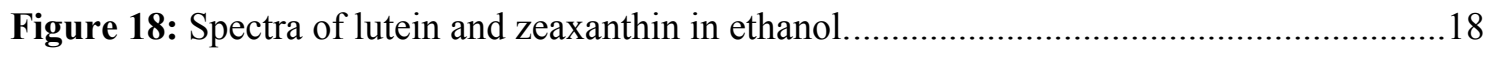


Figure 19: Scheme of possible pathway for the uptake, transport, and accumulation of the macular carotenoids in the human retina.

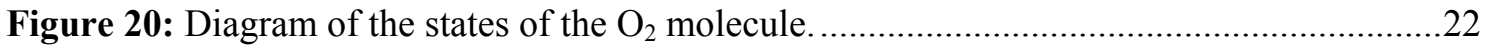

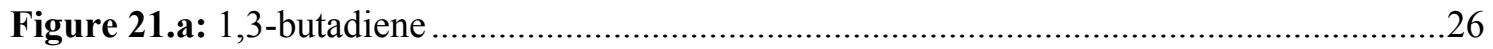

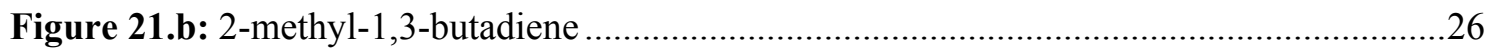

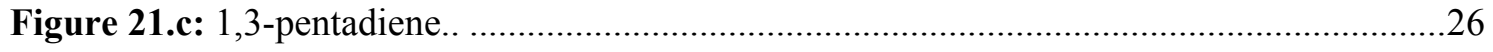

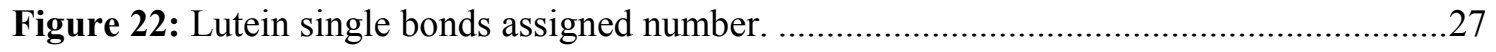

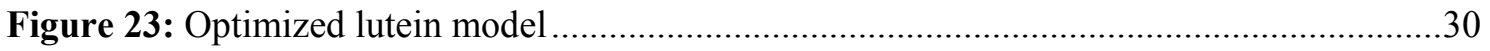

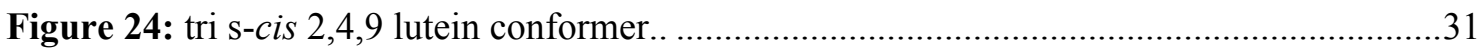

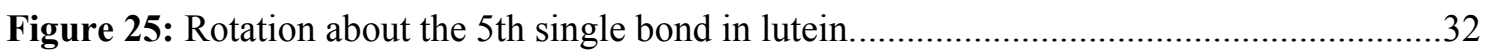

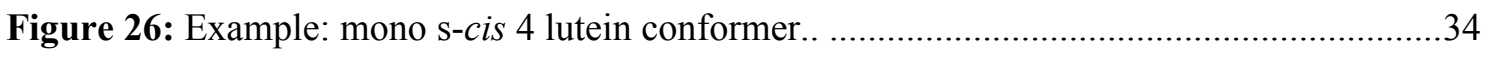

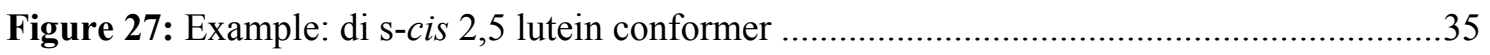

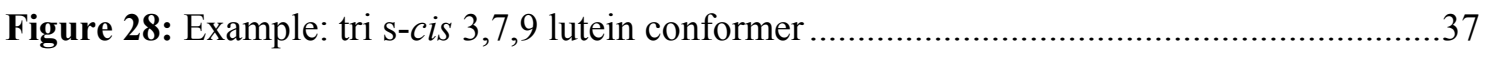

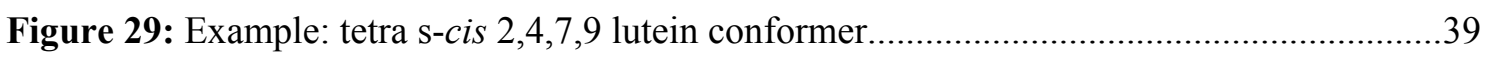

Figure 30: Example: penta s-cis 2,4,6,7,8 lutein conformer. ..............................................42

Figure 31: Example: hexa s-cis 3,4,5,6,7,8 lutein conformer. ............................................44

Figure 32: Example: hepta s-cis 1,3,4,5,6,7,9 lutein conformer ...........................................45

Figure 33: Example: octa s-cis 2,3,4,5,6,7,8,9 lutein conformer .......................................46

Figure 34: Example: nano s-cis 1,2,3,4,5,6,7,8,9 lutein conformer ......................................47

Figure 35: Energy barrier for the rotation of the single bond at the $4^{\text {th }}$ and $5^{\text {th }}$ bond in the lutein

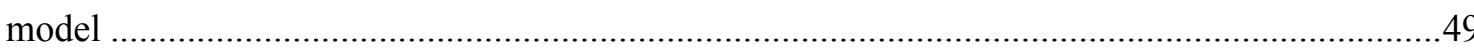

Figure 36: The energy of the lutein conformers according to the number of $s$-cis bonds and the their location within the molecule.

Figure 37: Correlation between the calculated B3LYP/6-31G* DE values and the predicted

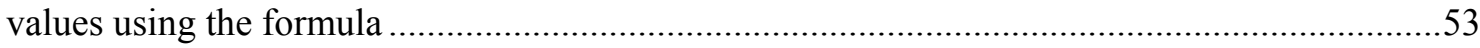

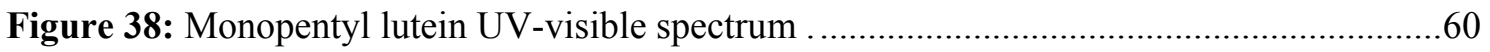

Figure 39: HPLC chromatogram of serum from subject before supplementation............61 
Figure 40: Expanded HPLC chromatogram of serum from subject before supplementation

Figure 41: Distribution of the carotenoids at baseline according to the group.................63

Figure 42: Comparison of carotenoids concentration in placebo group P1 ..............................65

Figure 43: Comparison of carotenoids concentration in L1 group ..........................................66

Figure 44: Comparison of carotenoids concentration in L2 group .......................................67

Figure 45: Comparison of carotenoids concentration in P2 group. ..........................................68

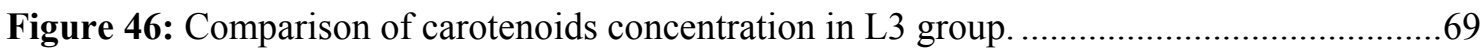

Figure 47: Comparison of carotenoids concentration in L4 group......................................70

Figure 48: Comparison of lutein concentration in serum for study I..................................

Figure 49: Comparison of lutein concentration in serum for study I......................................72

Figure 50: Average concentrations for lutein and zeaxanthin after supplementation in

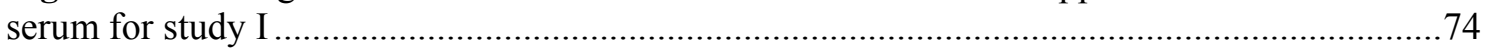

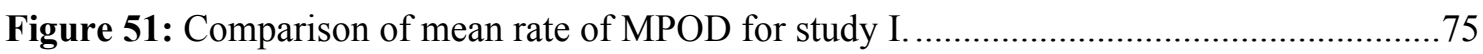

Figure 52: Comparison of lutein concentration in serum for study II ...................................... 76

Figure 53: Comparison of zeaxanthin concentration in serum for study II. ..............................77

Figure 54: Average concentrations for lutein and zeaxanthin after supplementation in

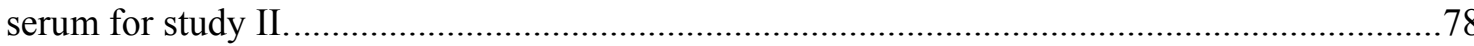

Figure 55: Comparison of mean rate of MPOD for study II..................................................79 


\section{LIST OF TABLES}

TABLE

PAGE

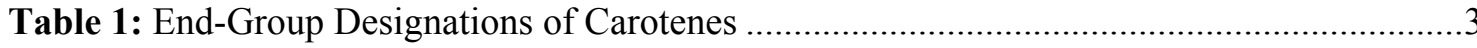

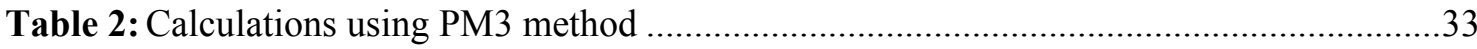

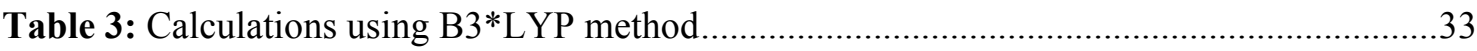

Table 4: Relative energies for conformers containing one $s$-cis single bond............................34

Table 5: Relative energies for conformers containing two $s$-cis single bonds ...........................34

Table 6: Relative energies for conformers containing three $s$-cis single bonds .......................35

Table 7: Relative energies for conformers containing three $s$-cis single bonds .........................36

Table 8: Relative energies for conformers containing three $s$-cis single bonds .........................36

Table 9: Relative energies for conformers containing three $s$-cis single bonds ............................

Table 10: Relative energies for conformers containing three $s$-cis single bonds ........................36

Table 11: Relative energies for conformers containing four $s$-cis single bonds ........................37

Table 12: Relative energies for conformers containing four $s$-cis single bonds .........................38

Table 13: Relative energies for conformers containing four $s$-cis single bonds ........................38

Table 14: Relative energies for conformers containing four $s$-cis single bonds ........................38

Table 15: Relative energies for conformers containing five $s$-cis single bonds............................

Table 16: Relative energies for conformers containing five $s$-cis single bonds........................40

Table 17: Relative energies for conformers containing five $s$-cis single bonds........................40

Table 18: Relative energies for conformers containing five $s$-cis single bonds .......................41

Table 19: Relative energies for conformers containing six $s$-cis single bonds ........................42

Table 20: Relative energies for conformers containing six $s$-cis single bonds .........................43

Table 21: Relative energies for conformers containing six $s$-cis single bonds .........................43

Table 22: Relative energies for conformers containing six $s$-cis single bonds ........................44

Table 23: Relative energies for conformers containing seven $s$-cis single bonds.....................44 
Table 24: Relative energies for conformers containing seven $s$-cis single bonds. .45

Table 25: Relative energies for conformers containing eight $s$-cis single bonds 46

Table 26: Relative energies for conformers containing eight $s$-cis single bonds .46

Table 27: Relative energies for conformers containing nine s-cis single bonds. .46

Table 28: Calculations with B ${ }^{*}$ LYP for selected conformers of the lutein model .47

Table 29: Calculations for dihedral angle for the $4^{\text {th }}$ and $5^{\text {th }}$ single bond in the lutein model $\ldots . .49$

Table 30: Values of the DE between $s$-cis and s-trans as calculated using PM3 and B3LYP/6$31 \mathrm{G}^{*}$

Table 31: Uv-vis data for carotenoids dissolved in ethanol ...................................................57

Table 32: Retention time of carotenoids in human serum .................................................62

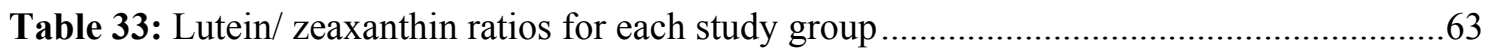

Table 34: Characteristic serum concentrations of carotenoids at baseline ...............................63

Table 35: Carotenoid concentrations in $\mathrm{P} 1$ throughout the study period (mean \pm standard deviation)

Table 36: Carotenoid concentrations after supplementation with L1 (mean \pm standard deviation) .65

Table 37: Carotenoid concentrations after supplementation with L2 (mean \pm standard deviation) .66

Table 38: MPOD rates for each group (mean \pm standard deviation) in study I

Table 39: Carotenoid concentrations after supplementation with $\mathrm{P} 2$ (mean \pm standard deviation)

Table 40: Carotenoid concentrations after supplementation with L3 (mean \pm standard deviation)

Table 41: Carotenoid concentrations after supplementation with L4 (mean \pm standard deviation)

Table 42: MPOD rates for each group (mean \pm standard deviation) in study II .71

Table 43: Concentrations for lutein and zeaxanthin in each group before and after supplementation in study I 
Table 44: Concentrations for lutein and zeaxanthin in each group before and after supplementation in study II. 


\section{CHAPTER 1: INTRODUCTION}

Carotenoids are pigments that are found in plants and microorganisms, but cannot be synthesized by animals [1]. These compounds are considered essential for life since they actively participate in the process of photosynthesis. They are responsible for the coloration in many flowers, fruits and vegetables. Carotenoids are also the principle pigments responsible for color in various animals: fish, birds' feathers and crustaceans [2]. Although more than 750 of these compounds are found in the nature and they are consumed regularly in the human diet only about 20 are detectable in human plasma and tissues [1].

\subsection{Carotenoid Structures}

Most carotenoids are tetraterpenoids and contain 8 isoprenoid units [3], Figure 1. Thus they have a $\mathrm{C}_{40}$ carbon skeleton. The carotenoid is built up by the linking of isoprenoid units in a head to tail pattern to form two $\mathrm{C}_{20}$ fragments that are linked together in a head to head fashion at the center. Consequently, at the center the two methyl groups of the polyene chain are six carbons apart from each other but they are only 5 carbons apart in other rest of the molecules.

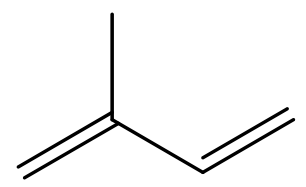

Isoprene unit

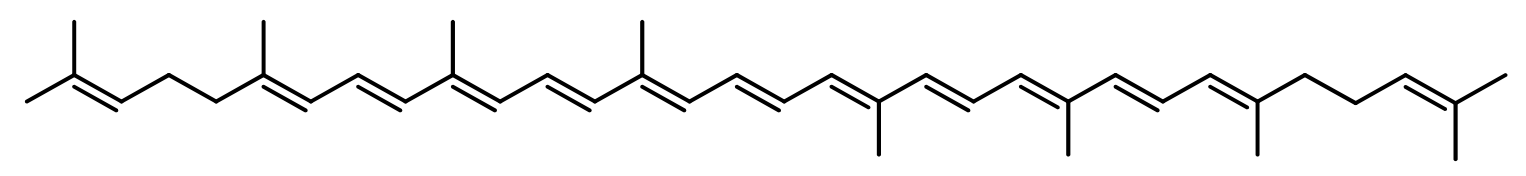

$\mathrm{C}_{40}$ Structure of Lycopene

Figure 1. Carotenoid structure 
$\mathrm{C}_{40}$ carotenoids are derived from lycopene by different synthetic pathways: cyclization, insertion of oxygen, double bond migration, methyl migration, chain elongation, and chain shortening [3].

Traditionally, carotenoids can be grouped into two classes: (a) carotenes or (b) xanthophylls. Carotenes are hydrocarbons (example: lycopene or $\beta$-carotene). Xanthophylls have oxygen atoms in their structure (in example: lutein and zeaxanthin).

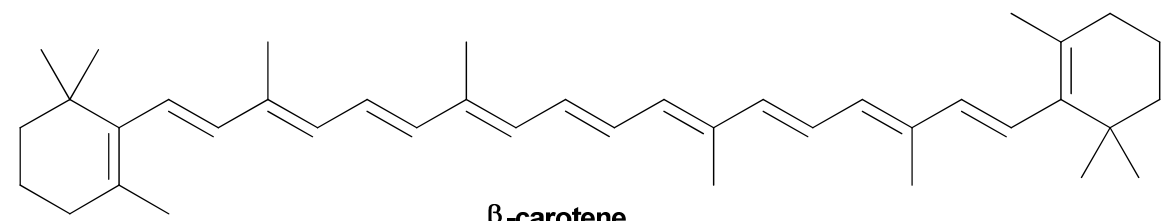

$\beta$-carotene
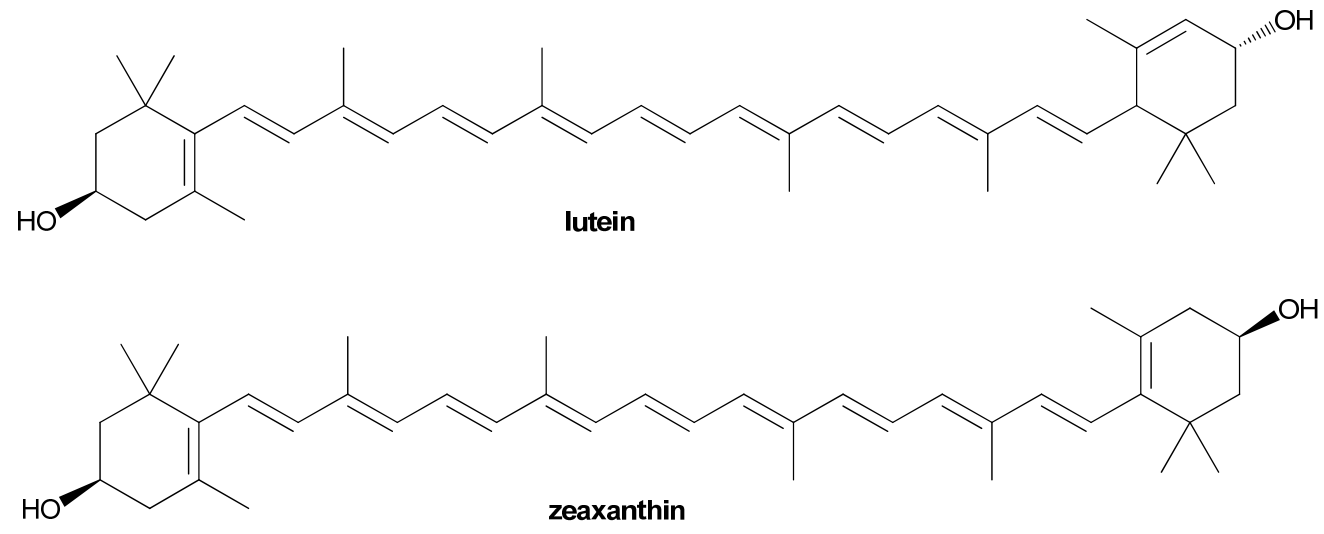

Figure 2. Carotene and Xanthophylls

For carotenes, all specific names are based on the stem name "carotene", which corresponds to the structure and numbering shown in Figure 3.

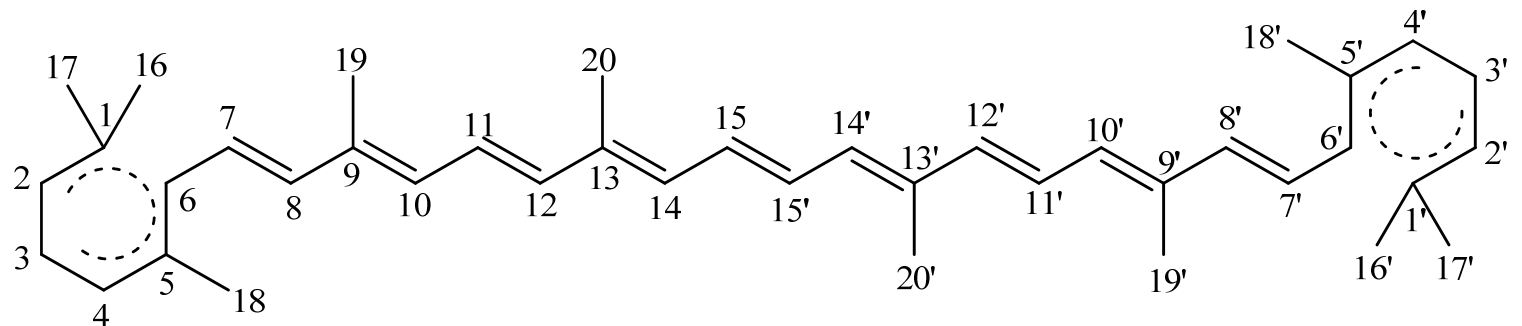

Figure 3. Carotene numbering system 


\subsection{Carotenoid End-Groups}

Structurally, the carotenoids possess end-groups that are either acyclic or a ring of five or six carbons at one or both ends of the molecule. Carotenes are named according to the end group(s) that they contain in their structures[3]. The end-groups and their prefixes are indicated in Table 1.

Table 1: End-Group Designations of Carotenes.

\begin{tabular}{|l|c|c|}
\hline \multicolumn{1}{|c|}{ Type } & Prefix & Structure \\
\hline Acyclic & $\psi$ & $(\mathrm{a})$ \\
\hline Cyclohexene & $\beta, \varepsilon$ & $(\mathrm{b}, \mathrm{c})$ \\
\hline Methylenecyclohexane & $\delta$ & $(\mathrm{d})$ \\
\hline Cyclopentene & $\kappa$ & $(\mathrm{e})$ \\
\hline Aryl & $\phi, \chi$ & $(\mathrm{f}, \mathrm{g})$ \\
\hline
\end{tabular}<smiles>[R]C=C(C)CCC=C(C)C</smiles>

(a) $\Psi$<smiles>[R]C1C(=C)CCCC1(C)C</smiles>

(d) $\gamma$

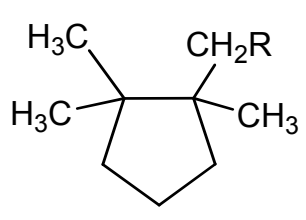

(e) $\kappa$<smiles>[R]C1=C(C)CCCC1(C)C</smiles>

(b) $\beta$<smiles>[R]c1c(C)ccc(C)c1C</smiles>

(f) $\phi$<smiles>[R]C1C(C)=CCCC1(C)C</smiles>

(c) $\varepsilon$

Figure 4.End-groups structure 
In green tissues, algae, and many fungi the major carotenoids are cyclic compounds such as: $\beta$-carotene and $\alpha$-carotene. The mentioned carotenes contain ionone rings in their structure. $\beta$ carotene has two $\beta$-ionone groups and $\alpha$-carotene has one $\varepsilon$-ionone and one $\beta$-ionone rings. In xanthophylls, which contain oxygen functional groups, the compounds are named according to the usual IUPAC nomenclature rules, indicating the position of the oxygen containing substituent. For example, the name of zeaxanthin (see Figure 2) is $\beta, \beta$-carotene-3,3'-diol.

\subsection{Biosynthesis $[3,4]$}

In plants, carotenoids are synthesized in the plastids by enzymes that are encoded by the nucleus. Carotenoids are built from the biological isoprene precursor isopentenyl pyrophosphate (IPP). The first specific terpenoid precursor is mevalonic acid (MVA). Mevalonic acid is converted into IPP by means of a three step enzymatic pathway; each step requires one mole of ATP per substrate. The first step is regulated by MVA kinase and this has been demonstrated in plant species and microorganisms. The identity of MVA-5-pyrophosphate, the product resulting from the second step, has been evidenced in yeast and has been identified as an intermediate in a bacterial system that can convert MVA into carotenes. Isopentenyl pyrophosphate can be found in enzymatic systems capable of carotenoid synthesis, like in tomato fruit.

To continue the chain elongation, in the first step IPP is isomerized to dimethylallyl diphosphate (DMAPP) by the enzyme IPP isomerase [5] (See Figure 6). The sequential addition of three IPP molecules to DMAPP, lengthens the chain to 10 carbons. Formation of the $\mathrm{C}_{15}$ molecular intermediate (FPP) is catalyzed by the enzyme geranylgeranyl diphosphate synthase (GGPS) that links DMAPP. The enzyme is one member of a closely related family of prenyl 
transferase enzymes that are distinguished by the length of the final product. The 20 -carbon molecule geranyl geranyl pyrophosphate (GGPP) is generated by two identical $\mathrm{C}_{5}$ addition steps.
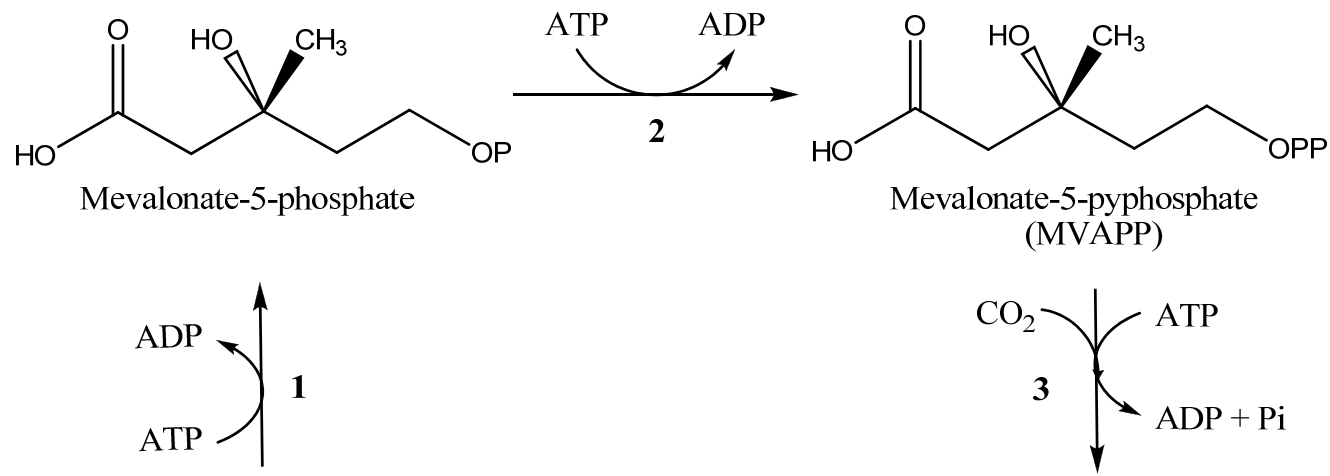
(MVAPP)

MVA
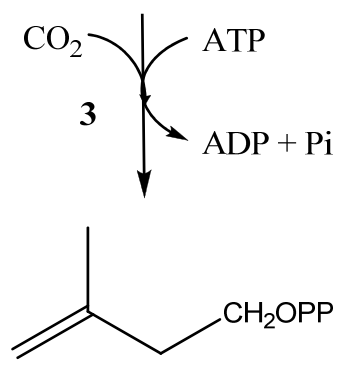

IPP

Figure 5. Formation of IPP from MVA

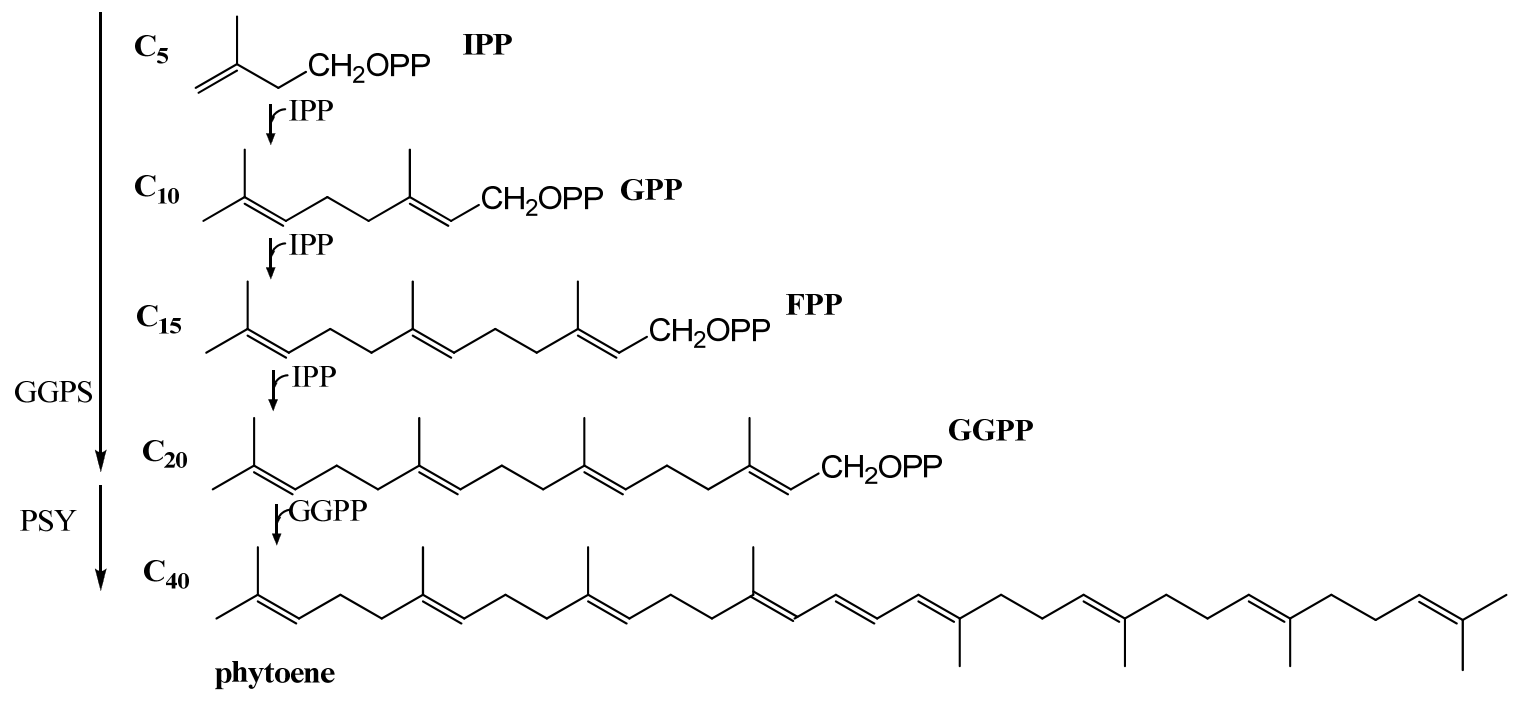

Figure 6. General pathway for the formation of phytoene [4]. The arrows on the side represent the enzymes involved. GGPS: geranylgeranyl diphosphate synthase. PSY: phytoene synthase. 
The next step in the carotenoid pathway is the condensation of two GGPP molecules to produce phytoene, which is catalyzed by a membrane-associated enzyme, phytoene synthase (PSY). Phytoene synthase shares amino-acid sequence similarity with GGPP synthase and other prenyl-transferases [5]. The condensation of two molecules of farnesyl pyrophosphate to produce squalene, the $\mathrm{C}_{30}$ precursor of sterols, is similar to the formation of phytoene from geranylgeranyl diphosphate synthase. In this biosynthesis pathway, NADPH is not required and one hydrogen is lost from $\mathrm{C} 1$ of each of the participating GGPP molecules. The GG residues are linked via sulphonium ylide.

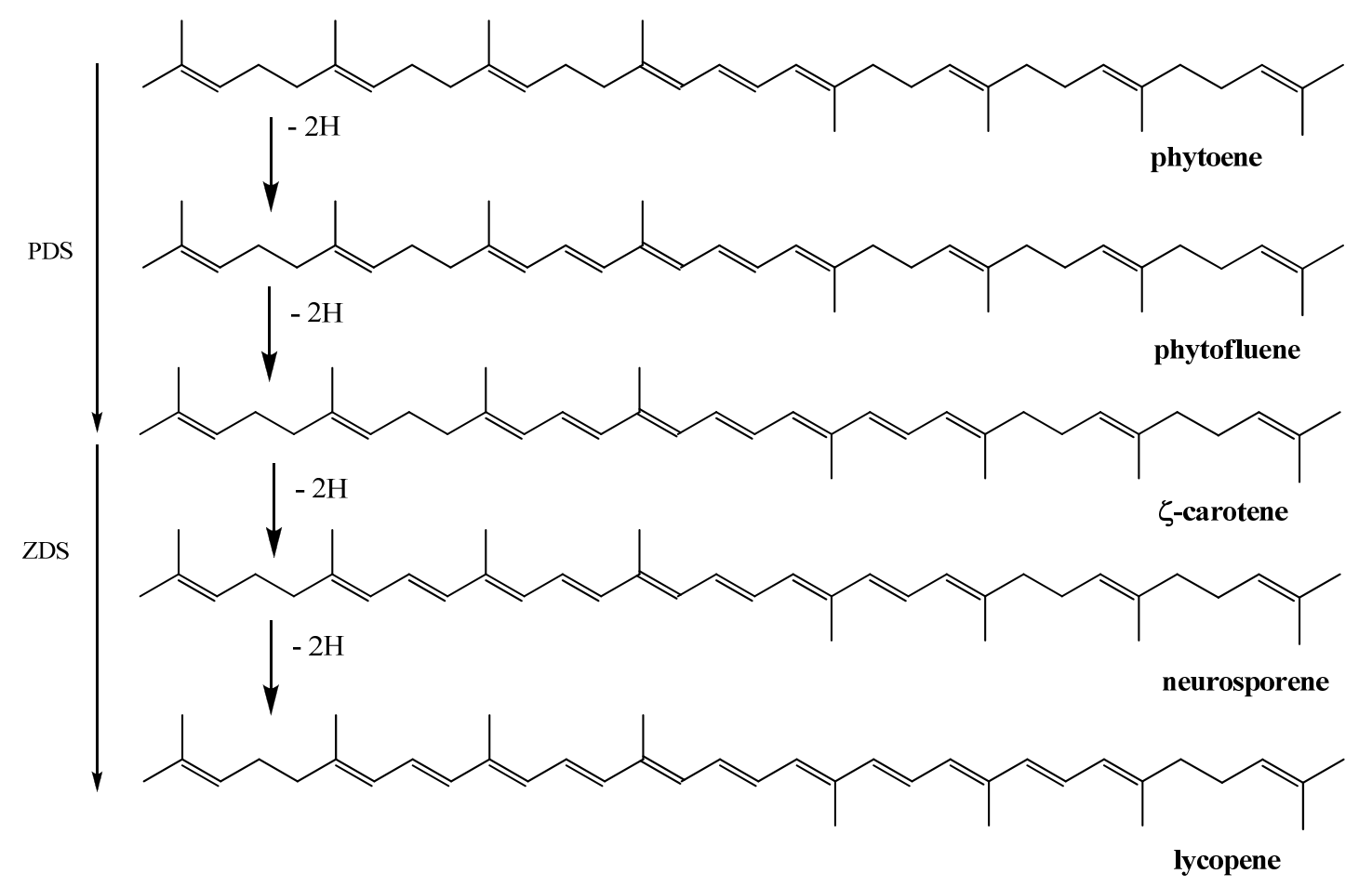

Figure 7. General pathway for the formation of lycopene [4]. The arrows on the side represent the enzymes involved. PDS: phytoene desaturase. ZDS: $\zeta$-carotene desaturase.

Four desaturation steps are required for the conversion of phytoene to lycopene and are catalyzed by two related enzymes in plants: phytoene desaturase and $\zeta$-carotene desaturase. 
However, bacteria and fungi achieve the same result via a single enzymatic complex. The first desaturation of phytoene produces phytofluene; then phytofluene undergoes dehydrogenation and isomeration to be converted into $\zeta$-carotene. $\zeta$-carotene is subsequently desaturated to produce neurosporene and lycopene. In the end the four reactions build the conjugated carbon backbone of lycopene.

At this point in the biosynthesis, the pathway branches and the cyclization of lycopene may occur to produce either $\beta$ - or $\varepsilon$-ring end-group structures. One branch will lead to the formation of $\beta$-carotene and its derivative xanthophylls, and the other branch leads to $\alpha$-carotene and lutein. $\varepsilon$-carotene is also produced from $\delta$-carotene.

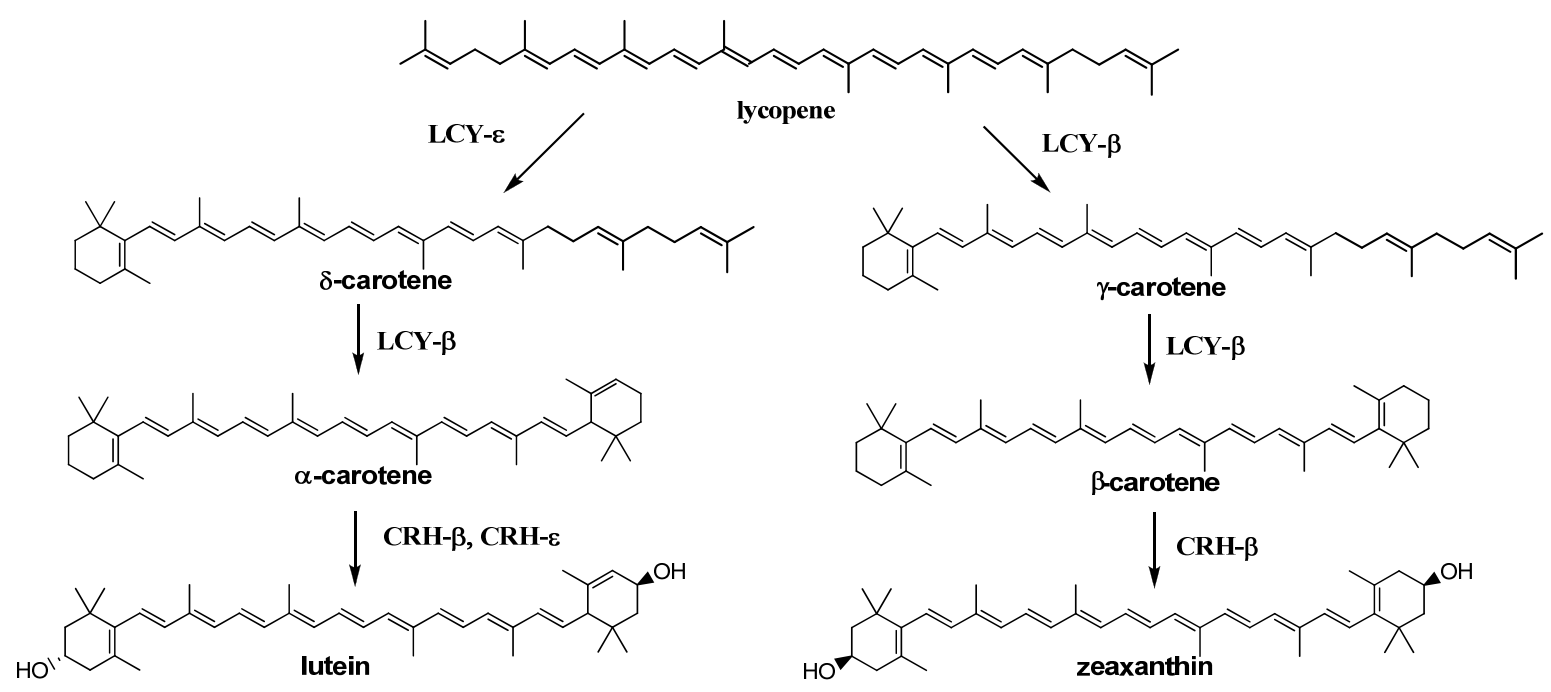

Figure 8. Cyclization of lycopene. Enzymatic conversions are shown by arrows with the enzymes responsible. LCY- $\beta$ : lycopene beta-cyclase; LCY- $\varepsilon$, lycopene epsilon-cyclase; CRH- $\beta, \beta$-carotene hydroxylase; $\mathrm{CRH}-\varepsilon$, epsilon-carotene hydroxylase [5].

During the biosynthesis of the cyclic carotenoids, the formation of $\varepsilon$ - and $\beta$-ionone rings take place at a late stage in the pathway. Experiments indicate that the $\beta$-ionone ring is formed by 
a mechanism that involves a carbonium ion (Figure 9). The cyclization concludes with the release of the $\mathrm{H}_{\mathrm{B}}$. proton from the C- 6 . $\varepsilon$-ionone is also formed by via a carbonium ion but in this case it is stabilized by expulsion of the $\mathrm{H}_{\mathrm{A}}$ proton at $\mathrm{C}-4$ [6].

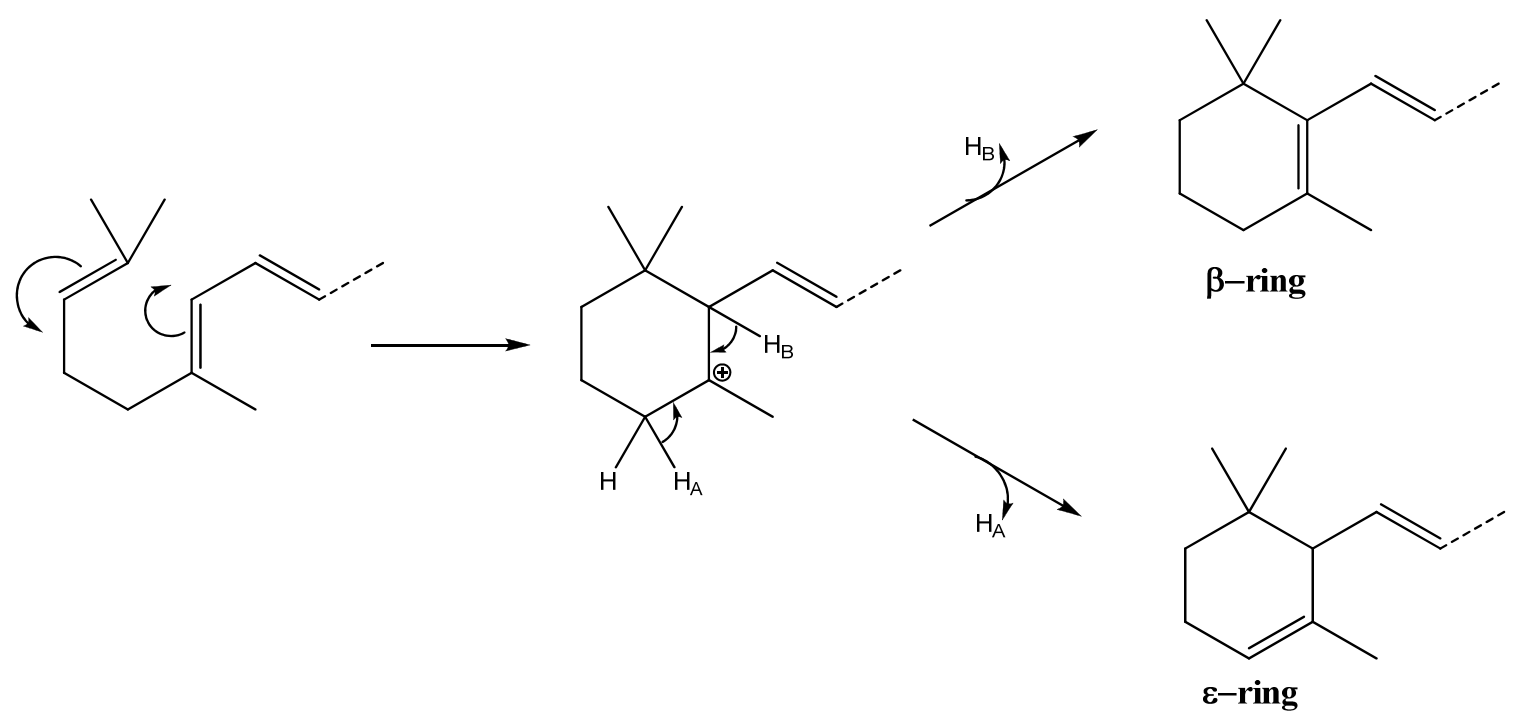

Figure 9. Basic mechanism for biosynthesis of $\varepsilon$ and $\beta$-rings in carotenoids

During the cyclization of lycopene, the reactions that are necessary for the formation of the carotenoid end-groups in both branches (the one that leads to lutein and the second that leads to zeaxanthin, Figure 8) are dependent on the relative activities of the cyclases involved in the reaction. The most common xanthophylls in the green tissue of higher plants, algae, and non-photosynthetic bacteria have hydroxyl substitutents at C-3, an oxo at C-4 or an epoxy at the 5, 6 position of the ionone ring [7]. Genetic evidence and functional analysis of an Arabidopsis $\beta$ hydroxylase enzyme support the existence of separate hydroxylases specific for the $\beta$ - and $\varepsilon$ rings. 


\subsection{Cis-carotenoids [8]}

The conjugated double bonds present in the structures of carotenoids give them the possibility to exist as several "cis-trans" geometrical isomers. Usually, we use cis and trans to indicate the orientation of substituent groups in a molecule relative to a double bond. When we have more than two different substituents on a double bond, $E$ and $Z$ are the more correct designations used to describe this geometrical isomerism. In nature, most carotenoids are found in the all-E (trans) configuration. It is also possible for the structures to have one or more than one "cis" or $Z$ double bond, but these are not energetically favored.

The $\beta$-carotene structure was elucidated by Karrer in 1930. In 1936, Gillam and El Ridi [9] reported in their study that when $\beta$-carotene was absorbed in an aluminium oxide column, it separates into two zones with different absorption maxima. They attribute this phenomenon to isomerization of $\beta$-carotene. With those studies the authors opened an unknown area in carotenoid research and from there more studies on cis and trans carotenoids were carried out. Further studies reported that in carotenoids other than $\beta$-carotene the same behavior was observed and more experimental data were presented to justify the conclusion that cis-trans isomerization was observed in all carotenoids.

As stated above, many geometrical isomers can potentially arise from a single molecule of a carotenoid. The number of stereoisomers in an "unsymmetrical" carotenoid (like lutein) with " $n$ " double bonds rises to $2^{\text {n }}$ possibilities. For "symmetrical" carotenoids, we have two cases: the first one, when " $n$ " is odd, the number of stereoisomers is $2^{(\mathrm{n}-1) / 2}\left(2^{(\mathrm{n}-1)}+1\right)$, for example $\beta$ carotene or violaxanthin. The second one, when " $n$ " is even, the number of stereoisomers is $\left(2^{(\mathrm{n} / 2)}\right.$ - 1) $\left(2^{(\mathrm{n} / 2)-1)}+1\right)$, for example rodhoxanthin. 


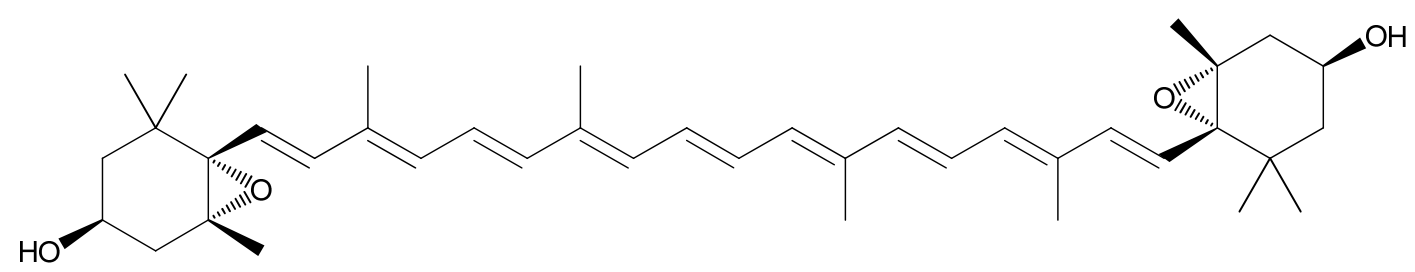

(a)

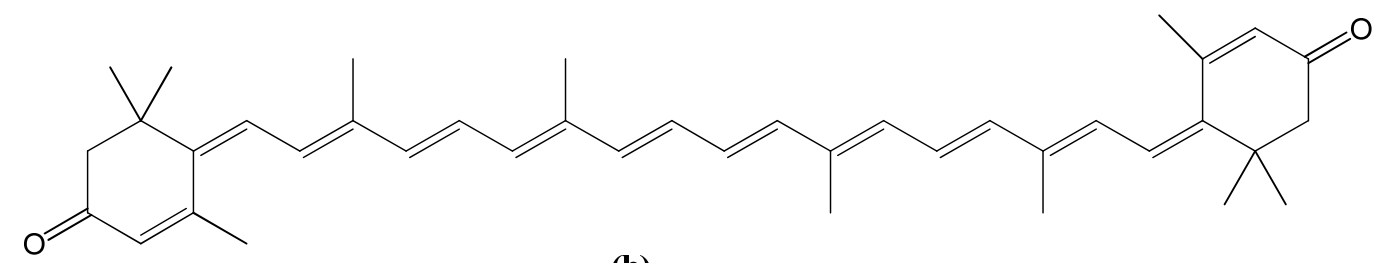

(b)

Figure 10. Examples of symmetrical carotenoids: (a) violaxanthin, (b) rhodoxanthin.

In practice, there is a difference between the number of theoretically calculated and experimentally observed steroisomers. Experiments show that each carotenoid, after treatment to produce a mixture of isomers, produces only a limited number of $c i$ isomers. The outcome of this reaction can be attributed to the relative energies of the all-trans (E) isomers compared to those in which a cis $(Z)$ geometry is present at some point in the polyene chain. The other important factor that must be considered is that proposed by Pauling. Pauling noted that isomerization can be favored as a result of overlapping of the methyl group of a carbon atom adjacent to a double bond and the hydrogen. We can explain this concept for carotenoids by looking at the backbone chain structure of the carotenoid, if we picture its orientation as seen in Figure 11: 


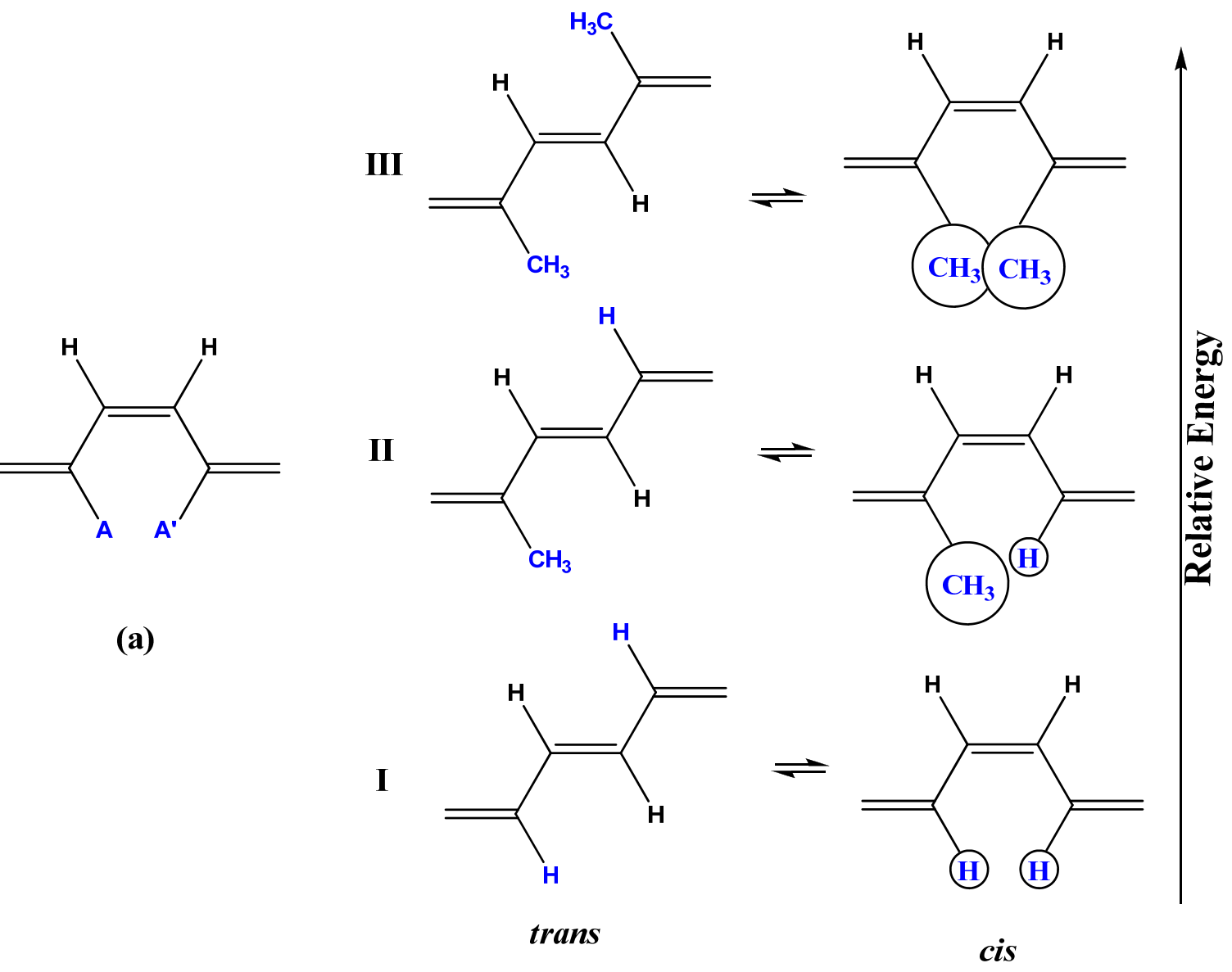

(b)

Figure 11. (a) Representation of a cis double bond in the polyene chain. (b) trans-cis cases

In this system A and A' may represent two hydrogen atoms, two methyl groups or one of each. If both A and A' are hydrogens (case I, Figure 11.b) the rotation around the central double bond will not meet any significant steric barrier in the planar cis geometry. However, if $\mathrm{A}$ is a methyl group and $\mathrm{A}^{\prime}$ is and hydrogen (case II, Figure 11.b) or another methyl group (case III, Figure 11.b) the cis geometry would have to overcome a larger energy barrier to exist.

Isomerization from trans to cis often occurs during chemical reactions, via a thermal or a light activated pathway, but may it also occur via reaction with an enzyme. Geometrical isomerization leads to a change of shape in the carotenoid molecule. The molecule shows bending 
and can form a zig-zag structure, see Figure 12. Presumably these shapes might have an important role in reactions with enzymes or with other proteins within the tissues.

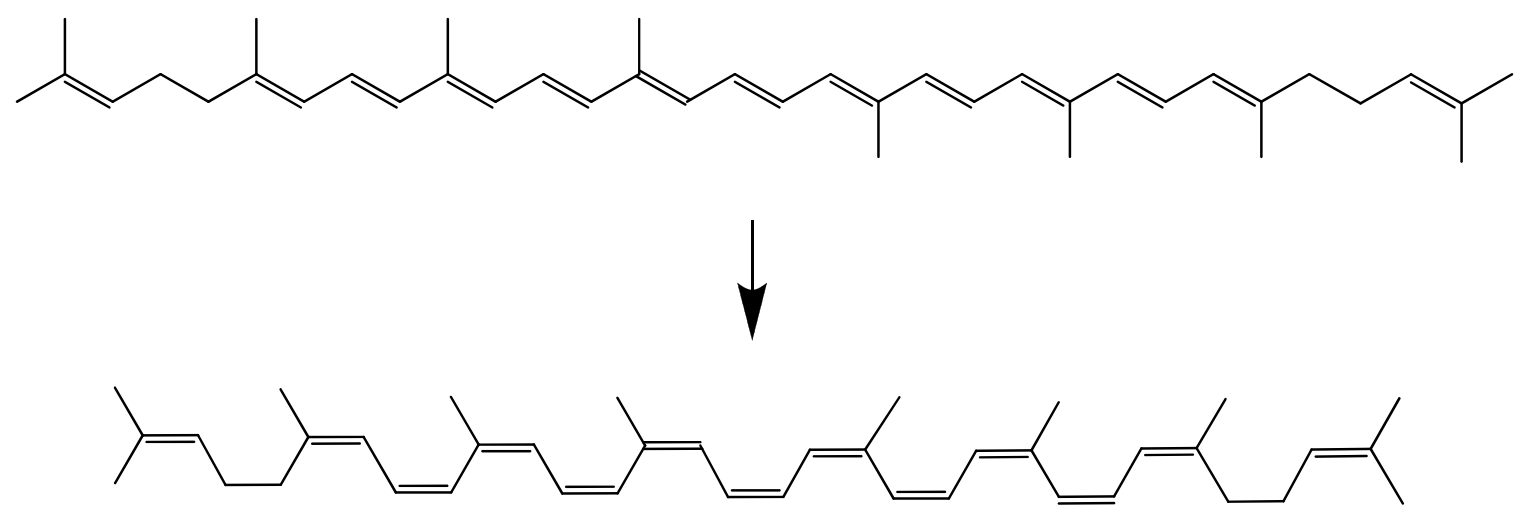

Figure 12. cis isomer of Lycopene

\subsection{Properties of cis carotenoids}

The following are some properties observed experimentally in cis isomers. The relative stability of an all-trans $(E)$ carotenoid is greater than that of cis isomers, because of the better electron delocalization and the more planar nature of the chromophore configuration.

According to Zechmeister's study; in an all-trans carotenoid solution, the carotenoid undergoes isomerization continually, first to monocis, next to dicis, and so on, during that process the structure star bending until a polycis solution is formed. The thermostability of the carotenoid solution first decreases during the isomerization, and during the process it gets to a point where the molecule is completely bent, because of its polycis configuration. In the end the structure accommodates in a compact overall shape so that the thermostability increases again. 


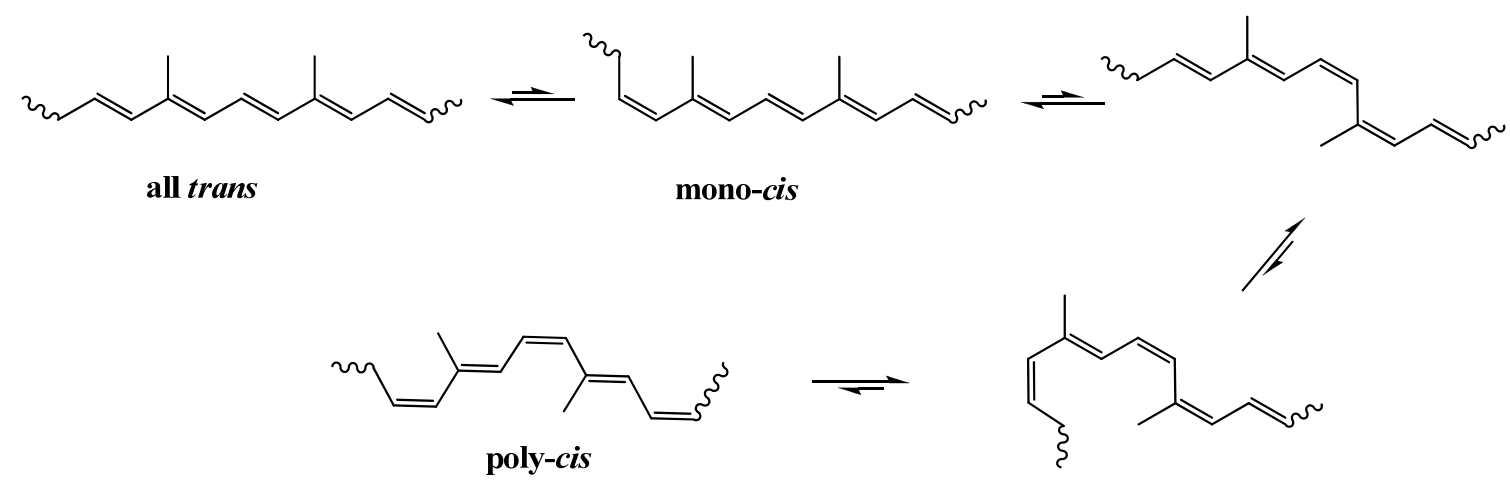

Figure 13. Scheme of isomerization of carotenoids.

Cis carotenoids are photochemically sensitive in the presence of iodine. Studies have shown that the all-trans isomer has the highest melting point of all the isomers. By comparison the cis conformers melt at lower temperatures. Interestingly, the decreasing of the melting point in a set of isomers is not proportional to the number of the cis bonds present in the molecule.

Spectral characteristics of all-trans carotenoids and mixtures of cis-trans carotenoids were analyzed by Zechmeister and Polgar [10]. Natural or synthetic carotenoids were treated by reflux for 45 minutes. The product was compared with an identical sample fraction that was treated with iodine for an hour at room temperature. It was observed that the color intensity decreased for the mixture of cis-trans isomers. The height and wavelength of the visible and ultraviolet maxima decreased in each case (Figure 14). 


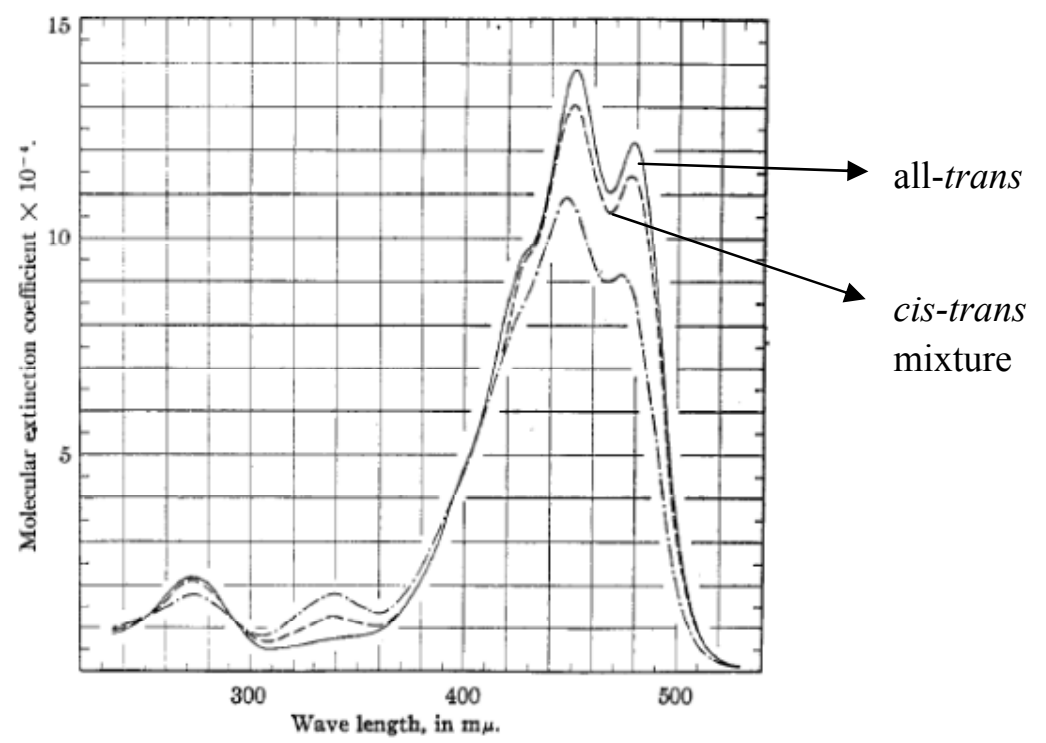

Figure 14. Molar extinction curves of $\beta$-carotene in hexane: all-trans solution. --- mixture of cis-trans isomers after refluxing. _._._mixture after iodine treatment [10].

\subsection{Naturally occurring cis carotenoid isomers.}

There are some carotenoids that have been identified in nature only as $Z$ isomers. Bixin was the first cis-polyene to be recognized in nature. It is isolated from annatto seeds (Bixa Orellana). Pro-lycopene was first detected to exist in the tangerine tomato in 194. The tangerine tomato has an orange color and accumulates pro-lycopene (7Z, 9Z, 7'Z, 9'Z-tetracis-lycopene) instead of the all-E-lycopene that is found in most tomatoes. Photoisomerization of pro-lycopene occurs in chloroplasts and is associated with the existence of the photosynthetic apparatus[11]. Studies have focused on trying to find a particular enzyme and/or look for possible environmental factors that can affect the isomerization of carotenoids in plants [11],[12]. But despite all of these efforts to understand the mechanism of carotenoid isomerization, it remains unclear how they are produced. Isomeric forms of lycopene can exist as the 5-cis, 9-cis, 13-cis and 15-cis isomers [13]. 


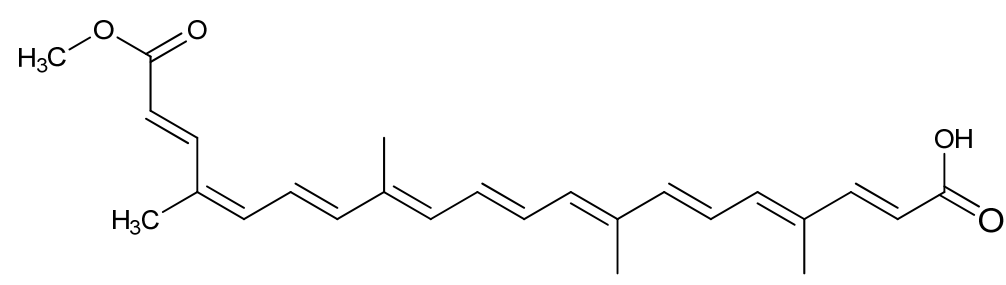

Bixin

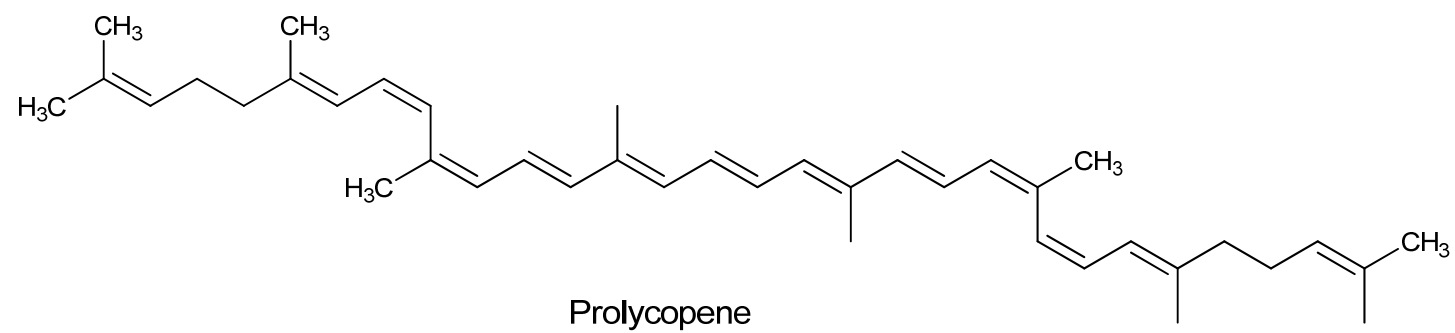

Figure 15. Natural cis carotenoids.

\subsection{Bioavailability of cis-carotenoids [14]}

Isomerization produces a significant difference in molecular structures that can affect the bioavailability of carotenoids. In tissues, approximately equal amounts of all- $E$ and various $Z$ lycopene isomers can be found. The $Z$ isomers have a greater solubility in mixed micelles and are less likely to aggregate or crystallize; as a result they more easily become incorporated into bile acid micelles, increasing the efficiency of their transport to tissues.

In fruits and vegetables $\beta$-carotene is found in the all- $E$ form and only small amounts of the $Z$ isomers are detected. The same is true for carotenoids in human plasma, but in some tissues $Z$ - $\beta$-carotene isomers accumulate to significant levels. These observations may be to the result of various factors such as a differential uptake of cis isomers, as well as differences in their absorption from the gut and transport in the human blood stream. Studies in gerbils after including $\beta$-carotene and vitamin $\mathrm{A}$ in the diet showed that the amounts of (9Z) - and (13Z)- $\beta$ carotene found in the liver were nearly equal to the amount of all- $E \beta$-carotene. An in vitro model of digestion showed that the incorporation of $Z$ isomers of $\beta$-carotene into micelles were 2-3 
times more efficient than the $E$ form. Lutein and zeaxanthin have been found in their cis isomers form: (13 Z)- and (13'Z)- lutein isomers; (9Z)- and (9'Z)- lutein; and (13-Z)- zeaxanthin in processed fruits, vegetables and pasta food products [15]. It has been demonstrated that trans-cis isomerization is an effect of the thermal processing of vegetables and fruits [16]. Studies $[17,18]$ have isolated the previous mentioned cis isomers of lutein and zeaxanthin (including 15Zzeaxanthin) in human serum and milk. Although the quantities isolated are lower in comparison with the all- $E$ isomers, it is shown that those xanthophylls isomers also exist in the human tissues.

\subsection{Macular Pigment Carotenoids (MPC)}

Lutein and zeaxanthin are two yellow carotenoids that give the macular area of the retina its color. The reason they are known as macular pigment carotenoids is that they are selectively accumulated in this part of the eye. The concentration of the macular pigment in the central retina approaches $1 \mathrm{mM}$, more than 1000 times that in human serum and liver[19].
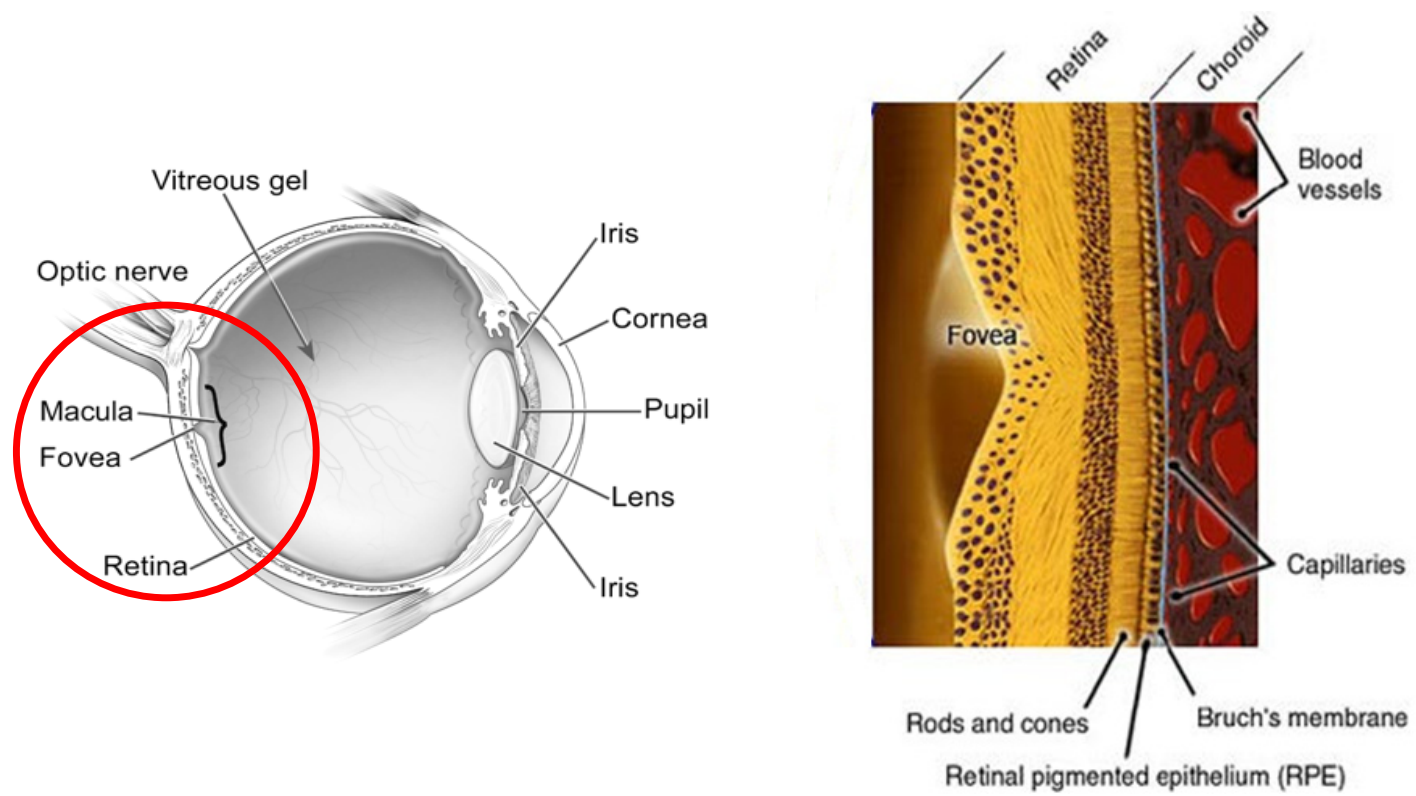

Figure 16. Representation of the human retina. 
The difference between these two molecules that compose the macular pigment is the location of one double bond in one of the end-groups and also the configuration of the hydroxyl group in that particular ring. Bone and Landrum showed that the major components of the macular pigment were lutein $\left[\left(3 \mathrm{R}, 3^{\prime} \mathrm{R}, 6^{\prime} \mathrm{R}\right)-\beta, \varepsilon\right.$-Carotene-3,3'-diol], zeaxanthin $\left[\left(3 \mathrm{R}, 3^{\prime} \mathrm{R}\right)-\beta, \beta-\right.$ Carotene-3,3'-diol] and meso-zeaxanthin $[(3 \mathrm{R}, 3$ 'S $)$ - $\beta, \beta$-Carotene-3,3'-diol][20]. Neither humans nor animals can synthesize carotenoids, so they must obtain them exclusively from their diet. Green leafy vegetables, fruits, and egg yolk are the primary sources of lutein and zeaxanthin available in nature.

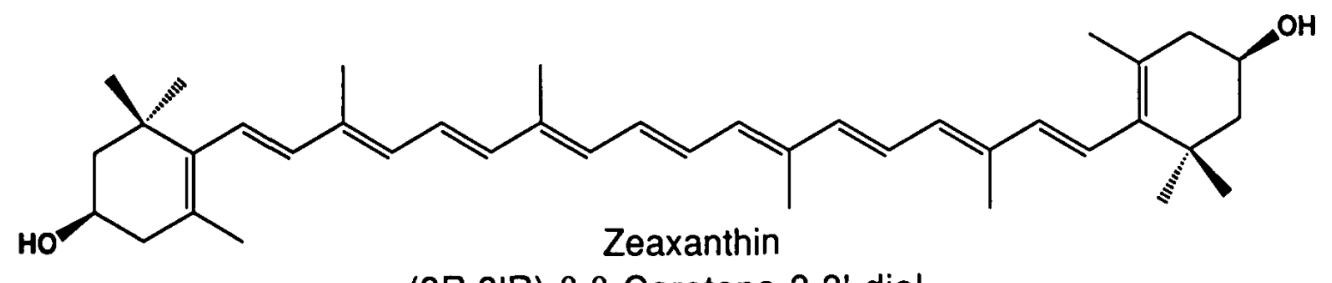

(3R,3'R)- $\beta, \beta$-Carotene-3,3'-diol

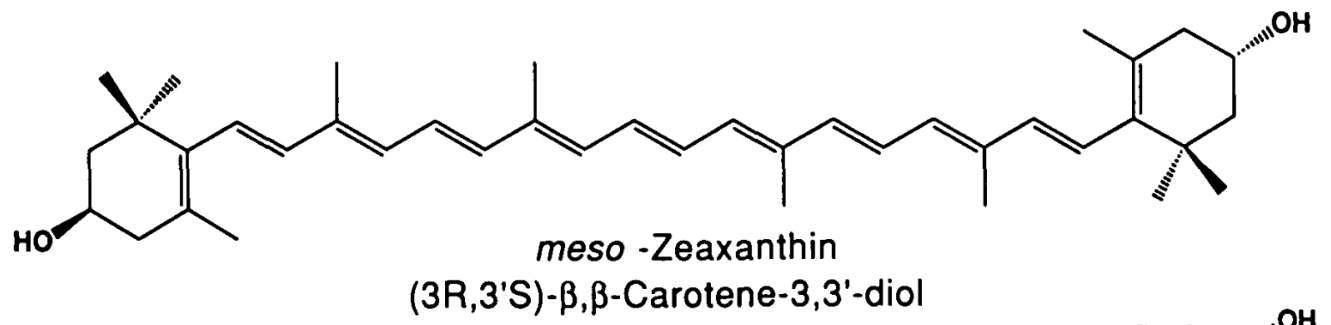

(3R,3'S)- $\beta, \beta$-Carotene-3,3'-diol

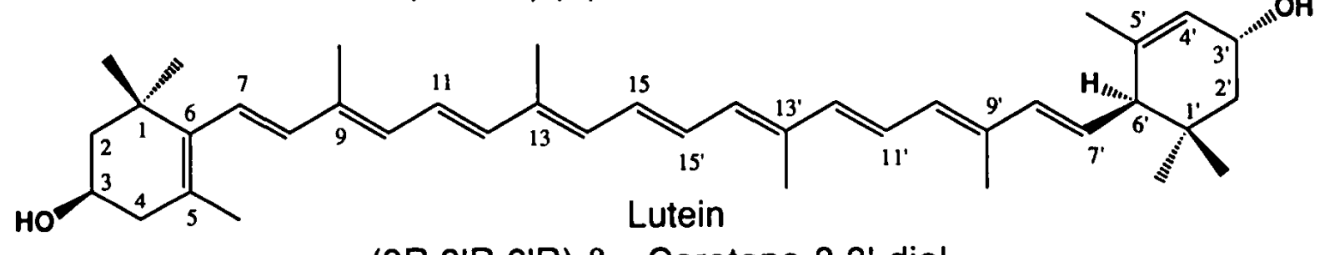

$\left(3 R, 3^{\prime} R, 6^{\prime} R\right)-\beta, \varepsilon$-Carotene-3,3'-diol

Figure 17. Major components of the macular pigment [20]

The content of lutein, zeaxanthin, and meso-zeaxanthin in the retina is approximately $36 \%, 18 \%$, and $18 \%$ respectively of the total carotenoid in the retina[21]. These carotenoids are 
concentrated in the fovea, which is the depression located in the retina coinciding with the position of maximum visual acuity.

Lutein and zeaxanthin can be characterized by their UV-visible spectra (Figure 18). The spectra may seem very similar but the presence of two $\beta$-ionone rings at the ends of the of the zeaxanthin structure extend the conjugation of the polyene chain and lower the energy separation between the ground and excited state, shifting its spectrum slightly to the red. Lutein absorbs at a slightly shorter wavelength than zeaxanthin.

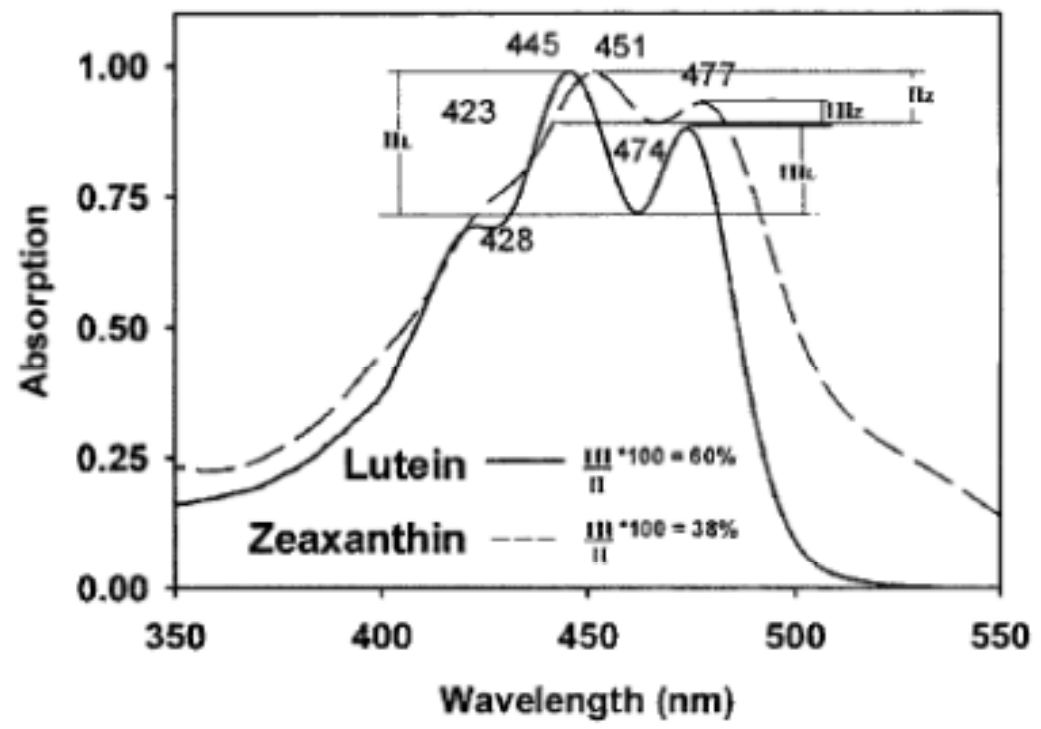

Figure 18. Spectra of lutein and zeaxanthin in ethanol[21].

On the basis of the spectra we can state that both carotenoids can filter blue light effectively. The retina is susceptible to oxidative stress because of the high demands for oxygen and its constant expose to light. A principal function of lutein and zeaxanthin as macular pigments appears to be the protection of the retina against photo-induced damage by acting as antioxidants in addition to their role as a filter for blue light. 


\subsection{Transport and distribution of MPC}

The absorption of lutein and zeaxanthin occurs in the enterocytes (intestinal absorptive cells) of the intestinal mucosa. They enter the hepatic portal circulation in the form of chylomicrons [22]. In the liver, low and high density lipoproteins (LDL, HDL) are synthesized and transport lutein and zeaxanthin to other tissues. It has been demonstrated that HDL is the primary carrier of lutein and zeaxanthin and that LDL is principally responsible for the transportation of carotenes[23]. Dietary lutein may be metabolized to produce meso-zeaxanthin within the retina, although the exact mechanism is still unknown[20]. A clear pathway of how these carotenoids are transported to the retina is still not well-defined. Li et al.[24] proposed a possible pathway for the uptake, transport and accumulation of these macular pigments carotenoids in the retina based on their studies on carotenoid-binding proteins. They suggest that the uptake of carotenoids within the cholesterol containing lipoproteins is mediated by HDL and HDL receptors, such as SR-BI (a cell surface glycoprotein that binds HDL). The mechanism would result in the delivery of lutein and zeaxanthin to the retinal pigment epithelium (RPE).

From the RPE a pathway analogous to the transport of retinoids within the retina is suggested for lutein and zeaxanthin. Accumulation of the MPC in the retina may be similar to a pathway known to deliver lutein to the silk glands in silkworms. In these larval insects, lutein is delivered to the silk gland via a pathway involving specific cell-uptake and specific binding proteins. 


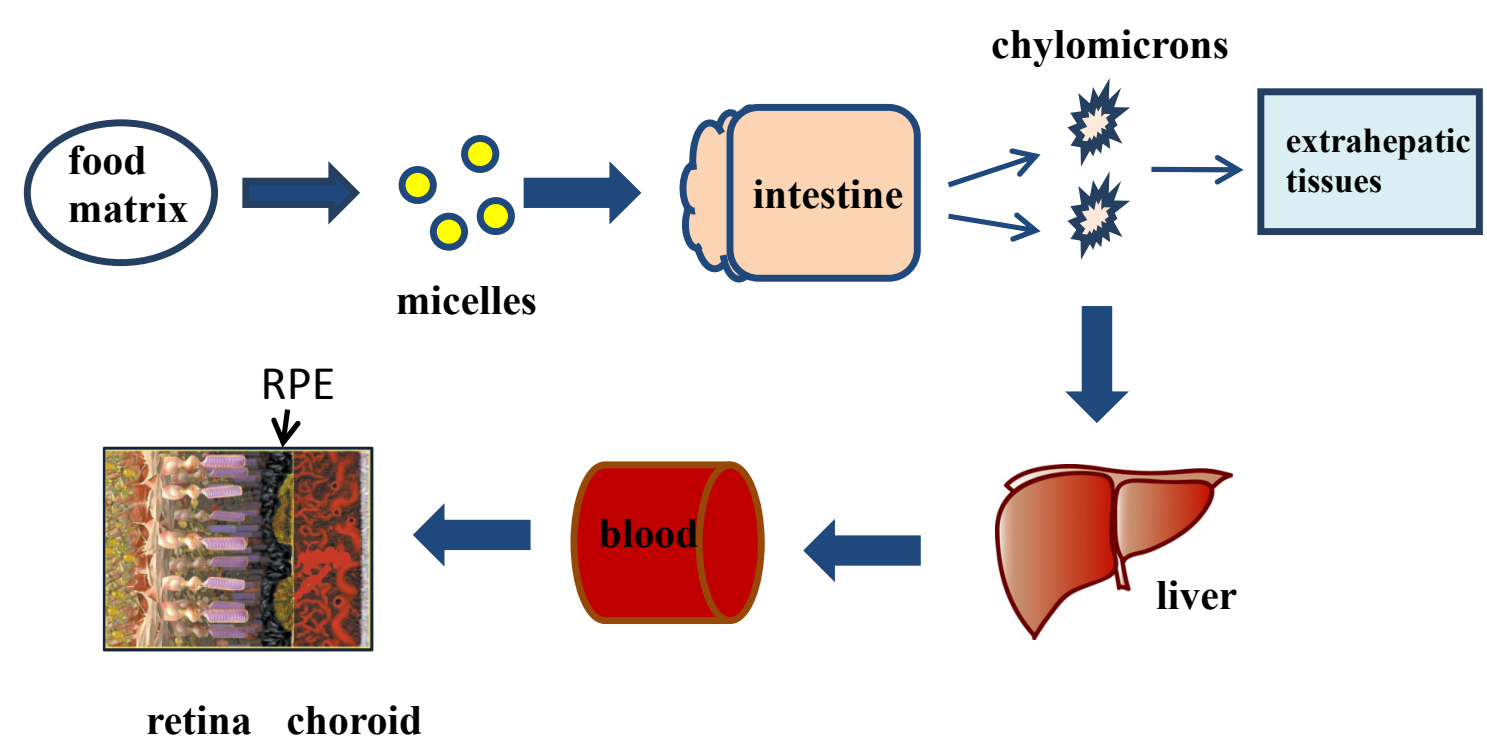

Figure 19. Scheme of possible pathway for the uptake, transport, and accumulation of the macular carotenoids in the human retina. RPE (retinal pigment epithelium).

As a general pathway for absorption and metabolism of carotenoids in animal models [25], it is known that after the consumption of carotenoids from the daily diet, these are incorporated in a complex food matrix with proteins and lipids, because of their lipophilicity. After the food matrix is digested, carotenoids will be combined with lipids and bile salts to form micelles. The micelles will move to the intestinal brush border membrane, and carotenoids will be captured and transported into the enterocyte to be metabolized and secreted into the intestinal lumen (cavity where the nutrients are absorbed) where they will be incorporated to chylomicrons and secreted into the lymph. Chylomicrons take the carotenoids to the liver where they can be processed by the liver and stored there or resecreted in VLDL (very low-low density-lipoprotein) and then to the bloodstream in the form of LDL and HDL. Carotenoids can also be captured by extra-hepatic tissues before they are taken to the liver. Lutein and zeaxanthin will be transported with HDL in the bloodstream, and the uptake of lutein and zeaxanthin on RPE cells will be mediated by the SR-B1 (scavenger receptor class B member 1, is a receptor for HDL) [24]. The 
selection of these carotenoids is probably determined by the selective binding proteins GSTP1 [24] (glutathione s-transferase P1) and StARD3 (steroidogenic acute regulatory domain protein 3) to mediate this process [26]. Experiments show that human GSTP1 exhibits a high affinity for zeaxanthin, and human StARD3 proteins bind lutein. Recently Bernstein's research group identify StARD3 as a lutein-binding protein in the macula of the primate retina[26]. Antibody labeling of retina cross-sectioned reveals that these proteins are found distributed in the same structures as the macular pigment.

\subsection{Age-Related Macular Degeneration (AMD)}

Age-Related Macular Degeneration is a disease that affects the central visual acuity. It is associated with age and is the leading cause of blindness in people over 65 years old [27]. AgeRelated Macular Degeneration is associated with abnormalities in the retina, degeneration of the RPE, and ultimately loss of the photoreceptors. Low levels of macular pigments appear to be a risk factor for AMD [28]. Studies report that high carotenoid intake is associated with a $43 \%$ risk reduction for exudative AMD. Lutein and zeaxanthin have been shown to be the most strongly associated, among all the carotenoids studied, capable of reducing the risk for AMD [29]. The AREDS $\mathrm{N}^{\circ} 22$ reported that participants with highest dietary intake of lutein and zeaxanthin were half as likely to have an advanced AMD as those with the lowest intake [30].

One of the principle reasons for our study of lutein and zeaxanthin is the properties that enable them to reduce the risk of AMD. A central characteristic of MPC is their ability to filter blue light. Blue light strikes the human retina and causes photooxidative damage to the lipid components of the photoreceptors disk membranes, which is a demonstrated characteristic of the pathology of $\mathrm{AMD}[21]$. 


\subsection{Principal properties of carotenoids [31, 32]}

As we mentioned above, carotenoids have an important role protecting cells and organisms against damage by light and reactive oxygen species (ROS) generated by light. The presence of conjugated double bonds in the structure of carotenoids is responsible for their most distinctive characteristic, light absorption [33].

Carotenoids can quench or inactivate the excited states of molecules. Krinsky explained how carotenoids can perform as antioxidants by reacting with active oxygen species in photobiological systems [32].

\section{Carotenoid quenching of singlet molecular oxygen $\left({ }^{1} \mathrm{O}_{2}\right)$}

The molecule $\mathrm{O}_{2}$ is required for all aerobic cell metabolism in nature. However, the excited state form of $\mathrm{O}_{2}$ singlet oxygen $\left({ }^{1} \mathrm{O}_{2}\right)$, is an unstable and highly reactive molecule particularly toward many biological substrates. The unstable form of oxygen can react with phospholipids compounds, that can be found in the biological cells [34]. Cell damage is frequently mediated by the formation of ${ }^{1} \mathrm{O}_{2}$ in photosensitized reactions. To explain how it is formed, it is helpful to understand the different states in the $\mathrm{O}_{2}$ molecule. In the ground-state, $\mathrm{O}_{2}$ has two electrons in two separate $\pi$ orbitals with their angular momentum opposed but with parallel spins. This ground-state is designated as the ${ }^{3} \Sigma$ because $\mathrm{O}_{2}$ exists as a triplet molecule and is a diradical. In the lowest excited state, ${ }^{1} \Delta$, the two electrons share a single orbital and they have the same spin. Since the electrons in this state are paired in accordance with the Pauli principle, the molecule is in a singlet state. $\mathrm{O}_{2}$ also has a second singlet state, ${ }^{1} \Sigma$. This state has a lifetime of only $1 \times 10^{-11} \mathrm{~s}$ and decays to the ${ }^{1} \Delta$ state. 


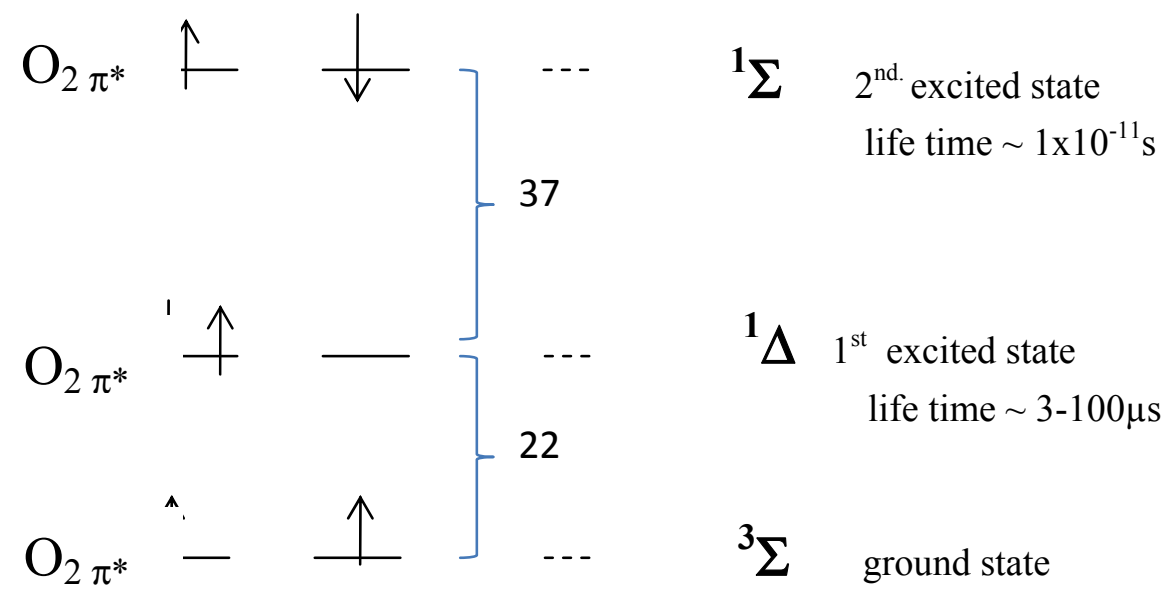

Figure 20. Diagram of the states of the $\mathrm{O}_{2}$ molecule.

When light strikes a biological system that contains an appropriate sensitizer (S) the energy of the photon can convert the sensitizer to an electronically excited form $\left({ }^{1} \mathrm{~S}\right)$. The singlet excited states for most molecular species are very short-lived, typically measured in nanoseconds $\left(<10\right.$ ns usually). By intersystem-crossing the singlet excited states, ${ }^{1} \mathrm{~S}$, can often be efficiently coverted to a longer lived triplet excited state, ${ }^{3} \mathrm{~S}$ (Equation 1).

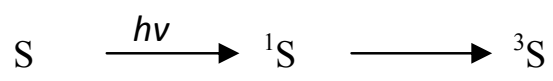

The ${ }^{3} \mathrm{~S}$ can initiate Norrish Type I or Type II photochemical reactions. In type I reactions the triplet sensitizer initiates radical reactions by abstracting an electron or hydrogen atom from nearby biomolecules creating reactive radical species (Equation 2). In the Type II reaction, the triplet sensitizer reacts with molecular oxygen. Since $\mathrm{O}_{2}$ is also a triplet species, formation of the highly reactive ${ }^{1} \mathrm{O}_{2}$ can occur renegerating the ground-state sensitizer (Equation 3), conserving the total spin of the system as required by quantum mechanics.

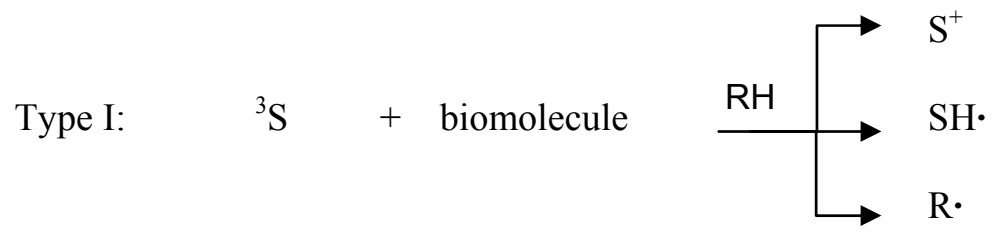


Type II: $\quad{ }^{3} \mathrm{~S}+{ }^{3} \mathrm{O}_{2} \longrightarrow{ }^{1} \mathrm{~S}+{ }^{1} \mathrm{O}_{2}$

Ground state carotenoid pi bonds (singlets) interact readily with ${ }^{1} \mathrm{O}_{2}$, accepting the excess energy from this reactive excited state. In this physical quenching of the oxygen excited state the carotenoid absorbs the energy from the ${ }^{1} \mathrm{O}_{2}$ and the result is the formation of the triplet state carotenoid (Equation 4). This reaction conforms to quantum mechanical restrictions that forbid a net change of spin state during any allowed process.

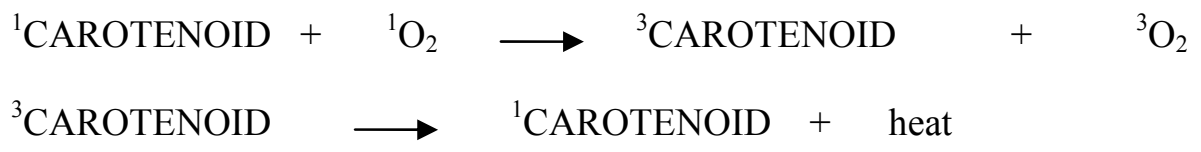

The presence of the conjugated polyene chain in the carotenoid enables these molecules to readily dissipate the energy through rotational and vibrational transitions and through collisions with the solvent liberating the excess energy harmlessly as heat. Thus the triplet carotenoid returns to its ground state unaltered (Equation. 5).

As we can see, the carotenoid may act as an antioxidant by neutralizing the potentially harmful ${ }^{1} \mathrm{O}_{2}$ molecule in tissues and cells. Our research is concentrated on lutein and zeaxanthin and its importance to living organisms.

In an effort to understand the nature of these two molecules in nature, and the uptake and transport of lutein and zeaxanthin in the human body we conducted two studies. The first is a theoretical study. We have cataloged the different possible conformations of the lutein molecule model resulting from s-cis geometries in the polyene chain. The goal of this study is to provide a better understanding of the possible interactions of carotenoids within the environment in the cell. The second part of this thesis addresses an applied project in which we studied the bioavailability 
of lutein and the relationship of the levels of six major carotenoids in human serum before and during supplementation with lutein. 


\section{CHAPTER 2: A CATALOG OF THE RELATIVE ENERGIES OF LUTEIN CONFORMATION POPULATIONS}

\subsection{Overview:}

Carotenoids are characterized by the presence of a polyene chain, typically having 9 conjugated double bonds, predominantly having an all-E (or all-trans) configuration. These structures can acquire different conformations because of the rotation around the single bonds. The IUPAC defines s-cis, s-trans conformation as: "the spatial arrangement of two conjugated double bonds about the intervening single bond is described as s-cis if syn-periplanar and s-trans if anti-periplanar" [35], see Figure 21.

(a)
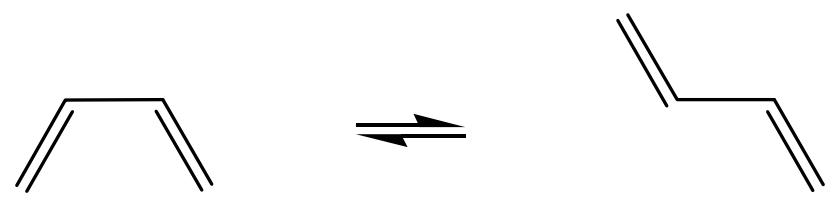

(b)
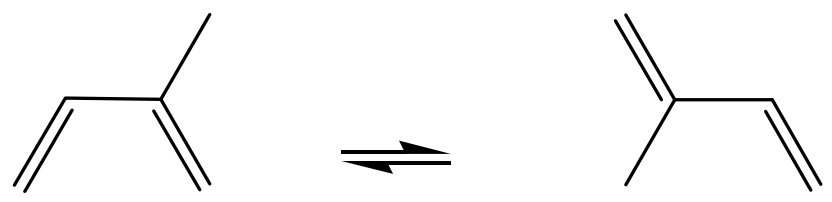

(c)
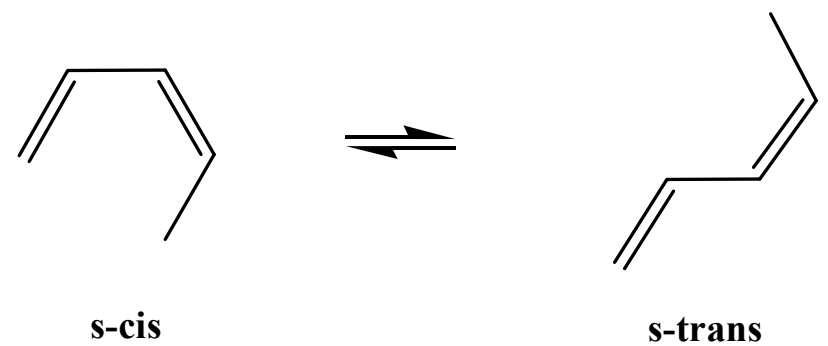

S-cis

s-trans

Figure 21. (a) 1,3-butadiene (b) 2-methyl-1,3-butadiene (c) 1,3-pentadiene.

The energetic barrier between the s-trans and s-cis conformations in simple conjugated systems like 1,3 butadiene is low. The enthalpy difference between the s-trans and s-cis was 
calculated in an ab initio study using the basis set $3-61 \mathrm{G}^{* *}$ and it was estimated to be about 16.26 $\mathrm{kJ} / \mathrm{mol}$ (3.89 $\mathrm{kcal} / \mathrm{mol})$ [36]. The classical model, for conjugated systems with methyl substitution, 2-methyl-1,3-butadiene is informative. The s-trans and s-cis conformers of 2methyl-1,3-butadiene are separated by a barrier estimated to be about $24.2 \mathrm{~kJ} / \mathrm{mole}(5.8$ $\mathrm{kcal} / \mathrm{mol})$.

The addition of a methyl group in the system on C-2 has little effect on the enthalpy difference between the s-cis and s-trans conformers for these butadienes (Figure 21). The conformational equilibrium between the $s$-cis and s-trans conformers of 2-methyl-1,3-butadiene (see Figure 21b) has been calculated to be $1.78 \pm 0.15 \mathrm{kcal} / \mathrm{mol}$ using an UV absorption spectroscopic method [37]. However, the ab initio study of the conjugated hydrocarbons, 2methyl-1,3-butadiene and 1,3-pentadiene (methyl at $\mathrm{C} 1$ of 1,3-butadiene) showed that the energy difference of the s-cis and s-trans conformer are $3.38 \mathrm{kcal} / \mathrm{mol}$ and $3.87 \mathrm{kcal} / \mathrm{mol}$ [38]demonstrating the importance of the position of the methyl to the energy of these conformers. The presence of methyl substituents on the carotenoid polyene chain and the more extensive conjugation compared to these simple dienes is expected to significantly alter the energetics of the accessible conformations and the barrier to inter-conversion between conformers [39].

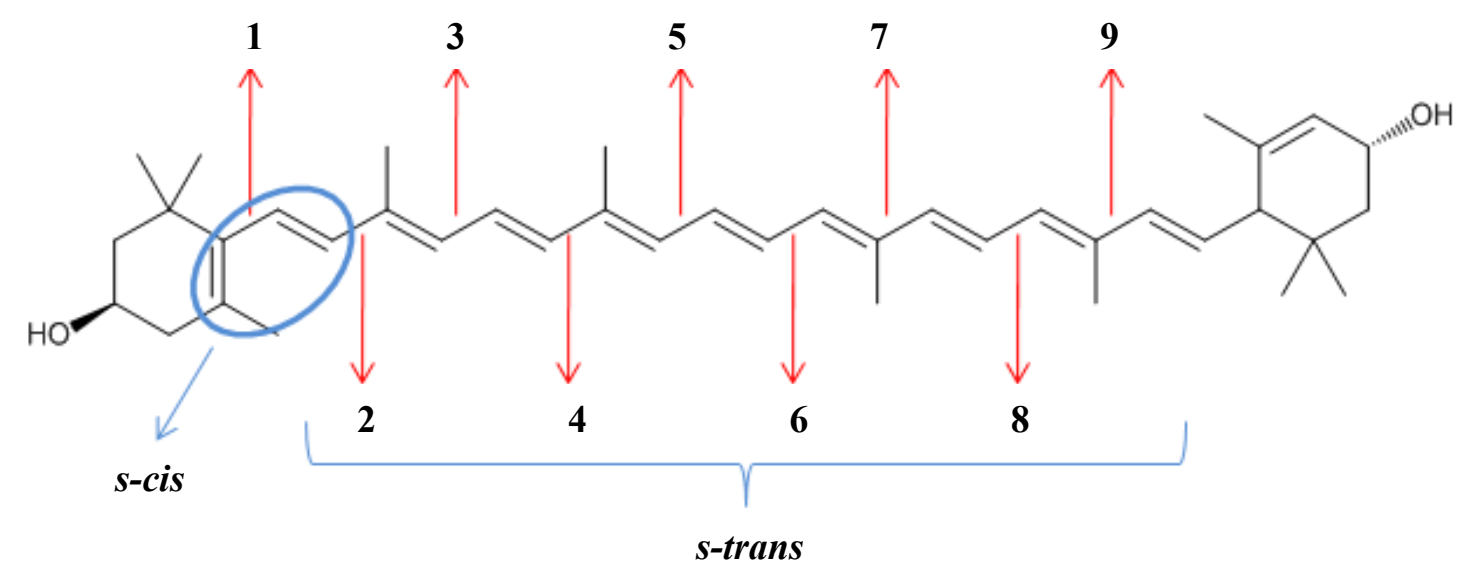

Figure 22. Lutein single bonds assigned numbers are shown by red arrows. 
The existence of 9 'single' bonds between the double bonds of the polyene chain (in lutein) makes possible the existence of numerous conformational geometries that correspond to rotation about these "single" bonds. In lutein, 512 conformations are possible, when considering the possible combinations of s-cis and s-trans conformations at the single bonds located along the backbone of the polyene chain.

In plants, fungi and invertebrates some carotenoid-binding proteins have been identified and characterized [40]. But in animals, carotenoid-binding proteins are still only poorly studied. Carotenoids in the circulation are transported in association with the lipoproteins. For example, the pathway that carotenoids like lutein and zeaxanthin follow to accumulate in the macula is not well-known. Serum lipoproteins and their receptors are known to be involved in the uptake and transportation of carotenoids in the humans and animals [41]. We can infer that the process of carotenoid binding to proteins requires dynamic mobility and their rapid interaction with the membrane proteins and enzymes within the photosynthetic apparatus of plants supports this conclusion. Since carotenoids can accumulate in cellular membranes, we also expect that their conformational flexibility will influence the rate and ability to repartition into other cellular organelles and proteins, both membrane-bound and water soluble. Thus, the affinity of carotenoids for binding to a protein and the rate of that process will be influenced by the energetic accessibility of conformations that favor the binding process. Carotenoids are often thought of as rigid, rod-like molecules, but they have a degree of flexibility that influences the dynamics and thermodynamics of their interaction with their environment. In this study, in an approach to develop a better understanding of how carotenoids interact with their environment and to highlight the importance of conformation in carotenoid binding, we conducted theoretical calculations on the energetics of conformations of the lutein. We calculated the minimum energy optimization profiles for all of the conformational geometries involving s-cis bonds. We created a 
catalog including all the different combinations of s-cis conformers for lutein. In order to accomplish this we have conducted our calculation using the complete carotenoid structure (as opposed to small molecule fragments) with the semi-empirical parametric method 3 (PM3)[42]. To validate the semi-empirical approach we have also performed a number of calculations using density functional B3LYP/6-31G* level of theory. We used the program Gaussian 09 Revision A.02 [43] for the B3LYP/6-31G* calculations. Semi-empirical methods are most useful for a comparative or qualitative understanding and have limitations that can invalidate their application in certain cases or influence the applicability of the calculation to particular systems.

Semi empirical methods, such as PM3, use parameters derived from experimental data to simplify the calculations and approximations needed to solve the Schrodinger equation. The PM3 is a level of theory that can be readily used for large molecules. In the past, PM3 has been used to calculate optimized 3-dimensional geometries and energy state descriptors of molecules in Quantitative Structure-Activity Relationship (QSAR) studies [44]. PM3 calculations are based on mathematical approximations. Density functional theory, on the other hand, is widely accepted to have better accuracy than semi-empirical methods. They compute electron correlation using general functions of electron density [45]. Becke's three-parameter hybrid functional (B3LYP) is a hybrid functional and is used to determine the electron density within a molecule. [45]. The B3LYP method has been proven to be a superior approach when compared with the semiempirical methods but is a time-consuming method. Density functional theory is more useful in that it can predict diverse molecular properties: vibrational frequencies, ionization energies and magnetic properties, etc. It has become more commonly used in computational chemistry over the past 10 years. 


\subsection{Research Methodology}

\subsubsection{Geometry optimization using the semi-empirical theory: PM3}

Geometry optimizations were calculated for lutein conformers using the program Hyperchem 8 [http://www.hyper.com/?tabid=360] Lutein was modeled in Hyperchem and the initial geometry was optimized to the minimum energy using the molecular mechanics $\left(\mathrm{MM}^{+}\right)$ force field method. Once an optimized geometry was obtained by the $\mathrm{MM}^{+}$method, the energy of the molecule was minimized using the semi-empirical PM3 calculation.

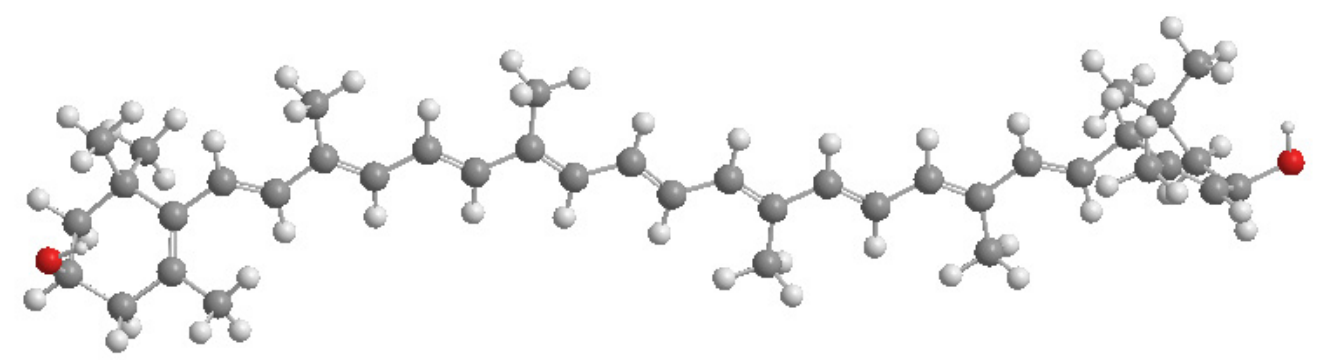

Figure 23. Optimized lutein model

After a geometry optimization of the all-trans structure was performed, we used that optimized lutein structure as the starting point to generate the various conformers by rotating around the single bonds in the molecule. The chosen bond(s) is set to $0^{\circ}$ (the planar s-trans geometry is $180^{\circ}$ and the s-cis is $0^{\circ}$ ). In the next step we allowed the calculation to find to the minimum energy of the geometry selected. For simplicity, in describing the various conformers, we number the nine single bonds within the polyene one through nine (see Figure 22) and name the conformers according to the single bond that has the s-cis conformation. For example, the conformer below is labeled tri-s-cis-2,4,9 because it is a molecule having three s-cis single bonds in the polyene system located at the $2^{\text {nd }}, 4^{\text {th }}$, and $9^{\text {th }}$ single bonds as numbered from left ( $\beta$-ring) to right ( $\varepsilon$-ring) (Figure 24). 


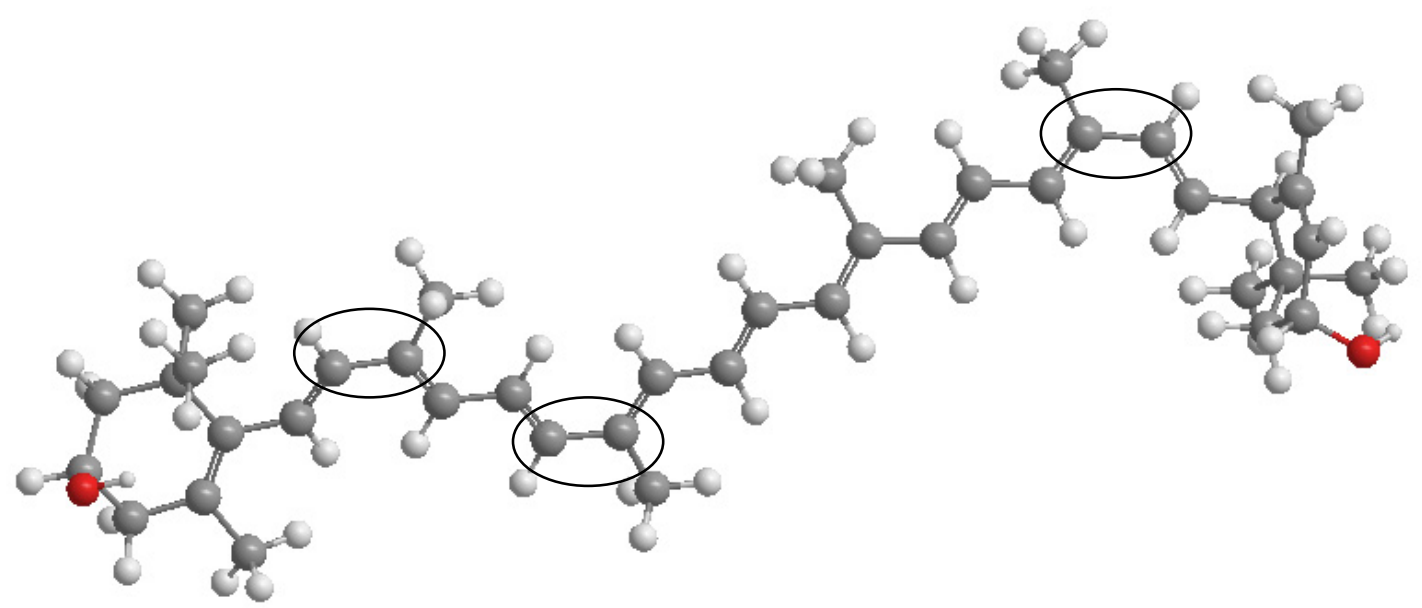

Figure 24: tri s-cis 2,4,9 lutein conformer.

The Tables numbered 4-27 are grouped according to the number of s-cis bonds present in the structures and provide a straight-forward way to group the data that resulted from our calculations. We have also created graphical representations that enable us to conceptualize a surface for each group of molecular conformers and recognize patterns that result from the placement of the s-cis bonds in different locations and/or combinations.

\subsubsection{Geometry optimization using the density functional theory: B3LYP/6-31G*.}

The results obtained from the PM3 calculations for some selected conformers were compared using the B3LYP/6-31G* computational method within the Gaussian 09 Revision A.02 program.

To run these calculations we used a Gaussian z-matrix format to specify the molecular geometry of the conformers. The z-matrix is derived by the Cartesian coordinates produced by the PM3 optimized geometries for the selected lutein conformers. The first line of the matrix, represented by two integers, specifies the charge and spin multiplicity of the molecule. The next lines indicate the atom, represented by its nominal atomic mass. And the location of the atoms is represented by the cartesian coordinates. 


\subsubsection{Calculation of the energy rotational barrier}

The conformers that were selected for validation using B3LYP were chosen on the basis of their energy patterns. A number of conformers had remarkably low energies as calculated by the PM3 method and validation of these was an important step. Similarly, some of the conformers had surprisingly higher energies and these too needed to be validated. Energy barriers for rotation around specific single bonds were calculated using the B3LYP/6-31G* method at the following torsion angles: $0^{\circ}, 30^{\circ}, 60^{\circ}, 90^{\circ}, 120^{\circ}, 150^{\circ}$, and $180^{\circ}$. A z-matrix was constructed for this purpose.

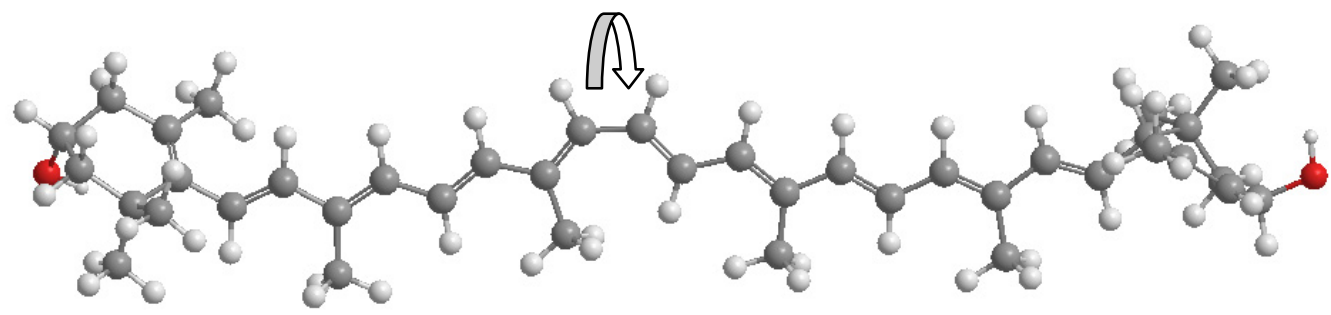

Figure 25: Rotation about the 5th single bond in lutein.

\subsection{Results}

\subsubsection{Calculations for the basic conjugated system}

Table 2 shows the calculations run for the s-cis and s-trans conformers of 1,3-butadiene, 2-methyl-1,3-butadiene, and 1,3-pentadiene (see Figure 21) using the PM3 method and Table 3 shows the calculations using B $3 *$ LYP theory. 
Table 2: Calculations using PM3 theory.

\begin{tabular}{|c|c|c|c|}
\hline PM3 & $\mathbf{1 , 3}$ butadiene & 2-methyl-1,3-butadiene & 1,3-pentadiene \\
\hline $\begin{array}{c}\text { s-cis } \\
\text { (kcal/mol) }\end{array}$ & -964.5380 & -1249.2777 & -1248.4794 \\
\hline $\begin{array}{c}\text { s-trans } \\
(\mathbf{k c a l} / \mathbf{m o l})\end{array}$ & -965.2665 & -1248.7766 & -1250.3684 \\
\hline$\Delta \mathbf{E ~} \mathbf{~ k c a l} / \mathbf{m o l}$ & 0.7285 & 0.5011 & 1.8990 \\
\hline
\end{tabular}

Table 3: Calculations using B3*LYP theory.

\begin{tabular}{|c|c|c|c|}
\hline B3*LYP & $\mathbf{1 , 3}$ butadiene & 2-methyl-1,3-butadiene & 1,3-pentadiene \\
\hline $\begin{array}{c}\text { s-cis } \\
(\mathbf{k c a l} / \mathbf{m o l})\end{array}$ & -98038.7021 & -122750.8496 & -122749.6427 \\
\hline $\begin{array}{c}\text { s-trans } \\
(\mathbf{k c a l} / \mathbf{m o l})\end{array}$ & -98042.5878 & -122754.5361 & -122754.1306 \\
\hline$\Delta \mathbf{E ~ k c a l} / \mathbf{m o l}$ & 3.8857 & 3.6865 & 4.4879 \\
\hline
\end{tabular}

The relative energies are compared for each of the three cases. The absolute energies calculated by the two methods cannot be compared because we are using two different theories. The semiempirical method only takes in account the valence electrons, while the density functional method uses all the electrons involved in the structure. This is why we can see a big difference between the values obtained in each case.

\subsubsection{Calculations for the complete carotenoid model using PM3.}

The following tables are grouped according to the number of s-cis bonds present in the structures. The tables indicate the s-cis location in the polyene chain (Figure 21) by the column and row respectively, and $\Delta \mathrm{E}$ is given in $\mathrm{kcal} / \mathrm{mol}$. For example: if the first column is numbered 2 , and the row 4 the conformer is di-cis 2,4 lutein. If the first column indicates 235 and the row 7 then the tetra-cis-2,3,5,7 lutein conformer is referred to. We have assigned the energy of the 
predominant in nature tobe zero. That conformer is the molecule corresponding to mono-cis 1lutein, notice that the $s$-cis bond is located at the beginning of the polyene chain (Figure 22,

Figure 23).

Tables 4-27 show the results obtained using the PM3 calculation.

Conformers containing one $s$-cis single bond

Table 4: Relative energies for conformers containing one $s$-cis single bond.

\begin{tabular}{|c|c|c|c|c|c|c|c|c|c|c|}
\hline $\begin{array}{c}\text { s-cis } \\
\text { location }\end{array}$ & $\mathbf{1}$ & $\mathbf{2}$ & $\mathbf{3}$ & $\mathbf{4}$ & $\mathbf{5}$ & $\mathbf{6}$ & $\mathbf{7}$ & $\mathbf{8}$ & $\mathbf{9}$ & $\begin{array}{c}\text { all- } \boldsymbol{E} \\
\text { lutein }\end{array}$ \\
\hline $\begin{array}{c}\Delta \text { Energy } \\
\mathbf{k c a l} / \mathbf{m o l}\end{array}$ & 0 & -0.7644 & 2.7274 & -0.6113 & 3.002 & 1.5183 & 0.1606 & 1.5491 & 0.3746 & 0.5387 \\
\hline
\end{tabular}

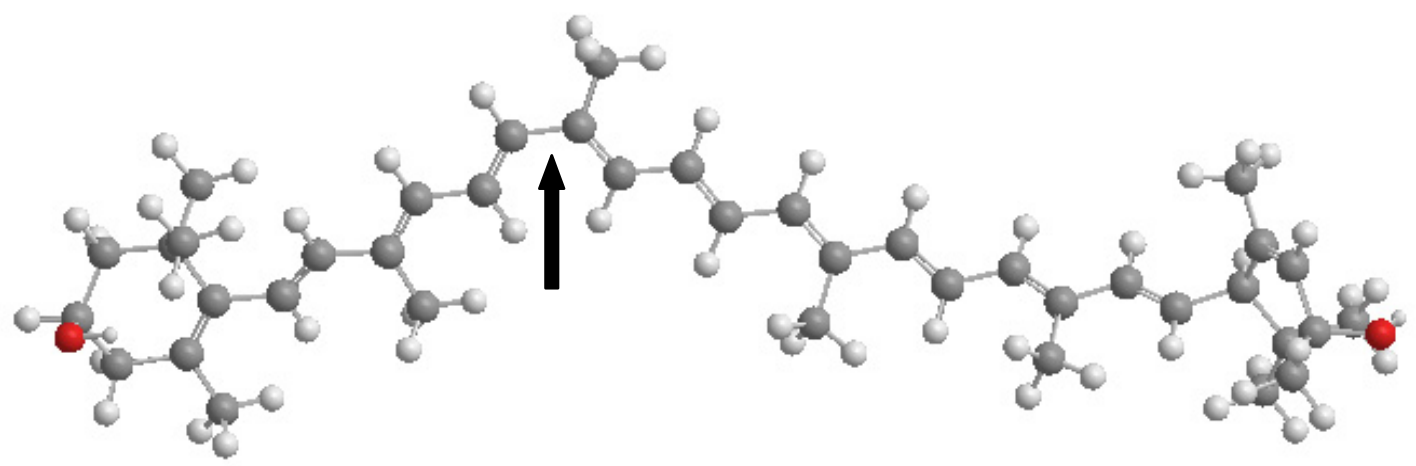

Figure 26: Example: mono s-cis 4 lutein conformer.

Conformers containing two $s$-cis single bonds

Table 5: Relative energies for conformers containing two $s$-cis single bonds.

\begin{tabular}{|r|r|r|r|r|r|r|r|r|}
\hline $\begin{array}{l}\text { s-cis } \\
\text { location/ } \\
\begin{array}{l}\Delta \mathbf{E} \\
\text { (kcal/mol) }\end{array}\end{array}$ & $\mathbf{2}$ & $\mathbf{3}$ & $\mathbf{4}$ & $\mathbf{5}$ & $\mathbf{6}$ & $\mathbf{7}$ & $\mathbf{8}$ & $\mathbf{9}$ \\
\hline $\mathbf{1}$ & 0.2374 & 2.1976 & -1.0314 & 2.4799 & 0.6376 & 0.2729 & 0.6117 & 0.4105 \\
\hline $\mathbf{2}$ & & 1.9600 & -1.8991 & 1.9954 & 0.2798 & -1.1254 & -0.0537 & -0.9086 \\
\hline $\mathbf{3}$ & & & 1.6728 & 5.1335 & 3.6838 & 2.4816 & 3.8212 & 2.6000 \\
\hline $\mathbf{4}$ & & & & 2.2157 & 0.5759 & -0.9789 & 0.5994 & -0.8073 \\
\hline
\end{tabular}


Table 5 continues:

\begin{tabular}{|r|r|r|r|r|r|r|r|r|}
\hline & $\mathbf{2}$ & $\mathbf{3}$ & $\mathbf{4}$ & $\mathbf{5}$ & $\mathbf{6}$ & $\mathbf{7}$ & $\mathbf{8}$ & $\mathbf{9}$ \\
\hline $\mathbf{5}$ & & & & & 4.2252 & -0.036 & 3.0676 & 1.315 \\
\hline $\mathbf{6}$ & & & & & & 1.1925 & 2.8143 & 1.3394 \\
\hline $\mathbf{7}$ & & & & & & & 2.7073 & -0.056 \\
\hline $\mathbf{8}$ & & & & & & & & 1.3619 \\
\hline
\end{tabular}

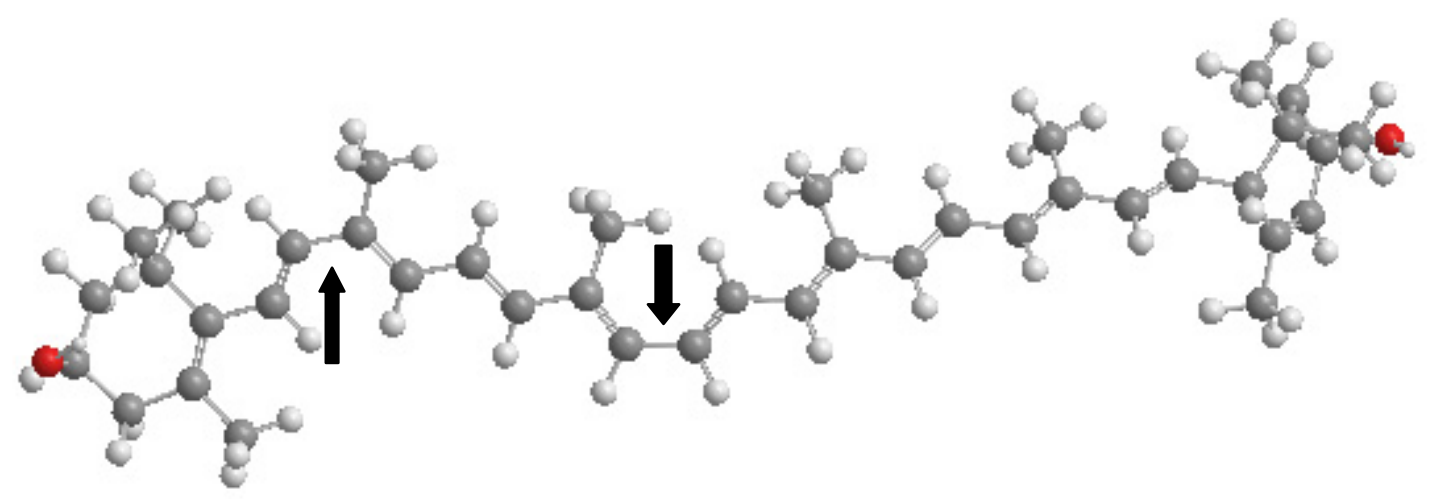

Figure 27: Example: di s-cis 2,5 lutein conformer.

Conformers containing three $s$-cis single bonds

Table 6: Relative energies for conformers containing three $s$-cis single bonds.

\begin{tabular}{|c|c|c|c|c|c|c|c|}
\hline $\begin{array}{l}\text { s-cis } \\
\text { location/ } \\
\Delta \mathbf{E} \\
\text { (kcal/mol) }\end{array}$ & 3 & 4 & 5 & 6 & 7 & 8 & 9 \\
\hline 12 & 2.1239 & -1.4354 & 2.4041 & 0.538 & -0.8199 & 0.5504 & -0.6838 \\
\hline 13 & & 1.5598 & 4.7342 & 2.8581 & 1.821 & 3.1677 & 1.9632 \\
\hline 14 & & & 1.7444 & 0.1922 & -1.3675 & 0.111 & -1.2193 \\
\hline 15 & & & & 3.5971 & 2.0585 & 2.4551 & 2.2535 \\
\hline 16 & & & & & 0.2948 & 2.0545 & 0.4807 \\
\hline 17 & & & & & & 1.5215 & 0.062 \\
\hline 18 & & & & & & & 0.4827 \\
\hline
\end{tabular}


Table 7: Relative energies for conformers containing three s-cis single bonds.

\begin{tabular}{|r|r|r|r|r|r|r|}
\hline $\begin{array}{r}\text { s-cis location/ } \\
\Delta \mathbf{E}(\mathbf{k c a l} / \mathbf{m o l})\end{array}$ & $\mathbf{4}$ & $\mathbf{5}$ & $\mathbf{6}$ & $\mathbf{7}$ & $\mathbf{8}$ & $\mathbf{9}$ \\
\hline $\mathbf{2 3}$ & 0.9026 & 4.4566 & 3.3257 & 1.7236 & 3.0586 & 1.8218 \\
\hline $\mathbf{2 4}$ & & 1.0048 & -0.6424 & -2.2371 & -0.6756 & -2.0602 \\
\hline $\mathbf{2 5}$ & & & 2.8725 & 1.6004 & 2.8764 & 1.7667 \\
\hline $\mathbf{2 6}$ & & & & -0.2303 & 1.4826 & 0.0477 \\
\hline $\mathbf{2 7}$ & & & & & 0.1396 & -1.3009 \\
\hline $\mathbf{2 8}$ & & & & & & 0.0362 \\
\hline
\end{tabular}

Table 8: Relative energies for conformers containing three $s$-cis single bonds.

\begin{tabular}{|r|r|r|r|r|r|}
\hline $\begin{array}{r}\text { s-cis location/ } \\
\Delta \mathbf{E}(\mathbf{k c a l} / \mathbf{m o l})\end{array}$ & $\mathbf{5}$ & $\mathbf{6}$ & $\mathbf{7}$ & $\mathbf{8}$ & $\mathbf{9}$ \\
\hline $\mathbf{3 4}$ & 5.0609 & 2.5288 & 1.3426 & 2.6144 & 1.5149 \\
\hline $\mathbf{3 5}$ & & 6.2409 & 4.7733 & 6.1011 & 4.9292 \\
\hline $\mathbf{3 6}$ & & & 3.0945 & 4.8855 & 3.5 \\
\hline $\mathbf{3 7}$ & & & & 4.0485 & 2.3182 \\
\hline $\mathbf{3 8}$ & & & & & 3.5441 \\
\hline
\end{tabular}

Table 9: Relative energies for conformers containing three $s$-cis single bonds.

\begin{tabular}{|r|r|r|r|r|}
\hline $\begin{array}{r}\text { S-cis location/ } \\
\Delta \mathbf{E}(\mathbf{k c a l} / \mathbf{m o l})\end{array}$ & $\mathbf{6}$ & $\mathbf{7}$ & $\mathbf{8}$ & $\mathbf{9}$ \\
\hline $\mathbf{4 5}$ & 2.455 & 1.757 & 2.9931 & 1.9936 \\
\hline $\mathbf{4 6}$ & & 0.0517 & 1.7433 & 0.3442 \\
\hline $\mathbf{4 7}$ & & & 0.3476 & -1.1515 \\
\hline $\mathbf{4 8}$ & & & & 0.2527 \\
\hline
\end{tabular}

Table 10: Relative energies for conformers containing three s-cis single bonds.

\begin{tabular}{|r|r|r|r|}
\hline $\begin{array}{r}\text { S-cis location/ } \\
\Delta \mathbf{E} \text { (kcal/mol) }\end{array}$ & $\mathbf{7}$ & $\mathbf{8}$ & $\mathbf{9}$ \\
\hline $\mathbf{5 6}$ & 3.8735 & 5.5269 & 4.0618 \\
\hline $\mathbf{5 7}$ & & 1.796 & -0.2219 \\
\hline $\mathbf{5 8}$ & & & 2.8145 \\
\hline $\mathbf{6 7}$ & & 2.55 & 1 \\
\hline $\mathbf{6 8}$ & & & 2.5598 \\
\hline $\mathbf{7 8}$ & & & 1.5593 \\
\hline
\end{tabular}




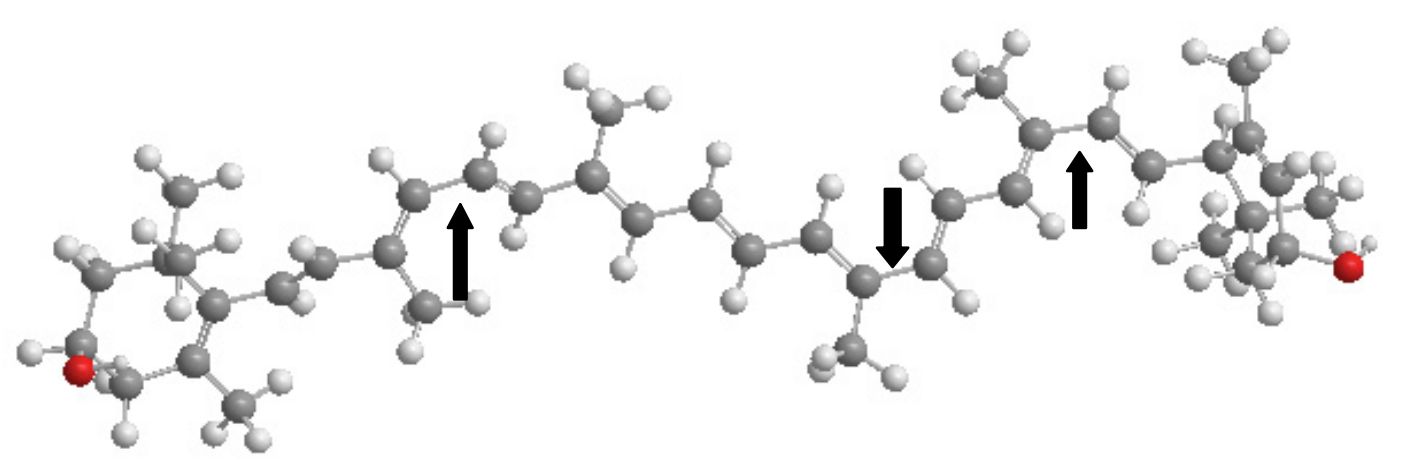

Figure 28: Example: tri s-cis 3,7,9 lutein conformer.

Conformers containing four $s$-cis single bonds

Table 11: Relative energies for conformers containing four $s$-cis single bonds.

\begin{tabular}{|c|c|c|c|c|c|c|}
\hline $\begin{array}{l}\text { s-cis } \\
\text { location / } \\
\Delta \mathbf{E} \\
\text { (kcal/mol) }\end{array}$ & 4 & 5 & 6 & 7 & 8 & 9 \\
\hline 123 & 0.891 & 4.3172 & 2.7676 & 1.4953 & 2.8415 & 1.6552 \\
\hline 124 & & 0.8519 & -0.7478 & -2.2994 & -0.8455 & -2.1818 \\
\hline 125 & & & 2.8691 & 0.2344 & 1.7645 & 1.6992 \\
\hline 126 & & & & -0.1139 & 1.444 & 0.0031 \\
\hline 127 & & & & & -0.0171 & -1.484 \\
\hline 128 & & & & & & -0.0298 \\
\hline 134 & & 4.5207 & 2.0724 & 0.5046 & 1.7316 & 0.8168 \\
\hline 135 & & & 5.3864 & 3.8934 & 4.6839 & 4.6046 \\
\hline 136 & & & & 2.5975 & 4.8767 & 3.3819 \\
\hline 137 & & & & & 3.6693 & 1.7748 \\
\hline 138 & & & & & & 2.4645 \\
\hline 145 & & & 2.6742 & 1.4906 & 2.752 & 1.5318 \\
\hline 146 & & & & -0.074 & 1.4571 & -0.0159 \\
\hline 147 & & & & & -0.0803 & -1.5653 \\
\hline 148 & & & & & & -0.0731 \\
\hline 156 & & & & 3.5572 & 4.1199 & 3.7065 \\
\hline 157 & & & & & 4.1322 & 1.0315 \\
\hline 158 & & & & & & 2.4942 \\
\hline 167 & & & & & 2.2851 & 0.6476 \\
\hline 168 & & & & & & 2.1906 \\
\hline 178 & & & & & & 0.804 \\
\hline
\end{tabular}


Table 12: Relative energies for conformers containing four $s$-cis single bonds.

\begin{tabular}{|r|r|r|r|r|r|}
\hline $\begin{array}{r}\text { s-cis location/ } \\
\Delta \mathbf{E}(\mathbf{k c a l} / \mathbf{m o l})\end{array}$ & $\mathbf{5}$ & $\mathbf{6}$ & $\mathbf{7}$ & $\mathbf{8}$ & $\mathbf{9}$ \\
\hline $\mathbf{2 3 4}$ & 3.4489 & 1.6584 & 0.5832 & 1.9288 & 0.729 \\
\hline $\mathbf{2 3 5}$ & & 5.3743 & 4.1745 & 5.3789 & 4.2424 \\
\hline $\mathbf{2 3 6}$ & & & 2.5971 & 4.4577 & 3.113 \\
\hline $\mathbf{2 3 7}$ & & & & 3.5382 & 1.5484 \\
\hline $\mathbf{2 3 8}$ & & & & & 2.796 \\
\hline $\mathbf{2 4 5}$ & & 1.7451 & 0.5239 & 1.7278 & 0.7652 \\
\hline $\mathbf{2 4 6}$ & & & -1.2147 & 0.4745 & -0.887 \\
\hline $\mathbf{2 4 7}$ & & & & -0.9227 & -2.4335 \\
\hline $\mathbf{2 4 8}$ & & & & & -0.9534 \\
\hline $\mathbf{2 5 6}$ & & & 2.5609 & 4.1602 & 2.684 \\
\hline $\mathbf{2 5 7}$ & & & & 2.7431 & 1.405 \\
\hline $\mathbf{2 5 8}$ & & & & & 2.6349 \\
\hline $\mathbf{2 6 7}$ & & & & 1.5175 & -0.4189 \\
\hline $\mathbf{2 6 8}$ & & & & & 1.2014 \\
\hline $\mathbf{2 7 8}$ & & & & & -0.0572 \\
\hline
\end{tabular}

Table 13: Relative energies for conformers containing four $s$-cis single bonds.

\begin{tabular}{|r|r|r|r|r|}
\hline $\begin{array}{r}\text { s-cis location/ } \\
\Delta \mathbf{E}(\mathbf{k c a l} / \mathbf{m o l})\end{array}$ & $\mathbf{6}$ & $\mathbf{7}$ & $\mathbf{8}$ & $\mathbf{9}$ \\
\hline $\mathbf{3 4 5}$ & 4.894 & 4.5533 & 5.7078 & 4.8054 \\
\hline $\mathbf{3 4 6}$ & & 2.01 & 3.5202 & 2.2922 \\
\hline $\mathbf{3 4 7}$ & & & 2.226 & 1.1479 \\
\hline $\mathbf{3 4 8}$ & & & & 2.3368 \\
\hline $\mathbf{3 5 6}$ & & 5.8798 & 7.3713 & 6.0545 \\
\hline $\mathbf{3 5 7}$ & & & 5.85172 & 4.5883 \\
\hline $\mathbf{3 5 8}$ & & & & 5.8524 \\
\hline $\mathbf{3 6 7}$ & & & 4.3631 & 2.8765 \\
\hline $\mathbf{3 6 8}$ & & & & 4.6341 \\
\hline $\mathbf{3 7 8}$ & & & & 3.5274 \\
\hline & & & &
\end{tabular}

Table 14: Relative energies for conformers containing four $s$-cis single bonds.

\begin{tabular}{|r|r|r|r|}
\hline $\begin{array}{r}\text { s-cis location/ } \\
\Delta \mathbf{E}(\mathbf{k c a l} / \mathbf{m o l})\end{array}$ & $\mathbf{7}$ & $\mathbf{8}$ & $\mathbf{9}$ \\
\hline $\mathbf{4 5 6}$ & 2.1081 & 3.4651 & 2.2871 \\
\hline $\mathbf{4 5 7}$ & & 2.8032 & 1.5687 \\
\hline $\mathbf{4 5 8}$ & & & 2.7528 \\
\hline $\mathbf{4 6 7}$ & & 1.6121 & -0.1375 \\
\hline
\end{tabular}


Table 14 continues:

\begin{tabular}{|r|r|r|r|}
\hline & $\mathbf{7}$ & $\mathbf{8}$ & $\mathbf{9}$ \\
\hline $\mathbf{4 6 8}$ & & & 1.4659 \\
\hline $\mathbf{4 7 8}$ & & & 0.1004 \\
\hline $\mathbf{5 6 7}$ & & 5.4027 & 3.6851 \\
\hline $\mathbf{5 6 8}$ & & & 5.2868 \\
\hline $\mathbf{5 7 8}$ & & & 1.4516 \\
\hline $\mathbf{6 7 8}$ & & & 2.2355 \\
\hline
\end{tabular}

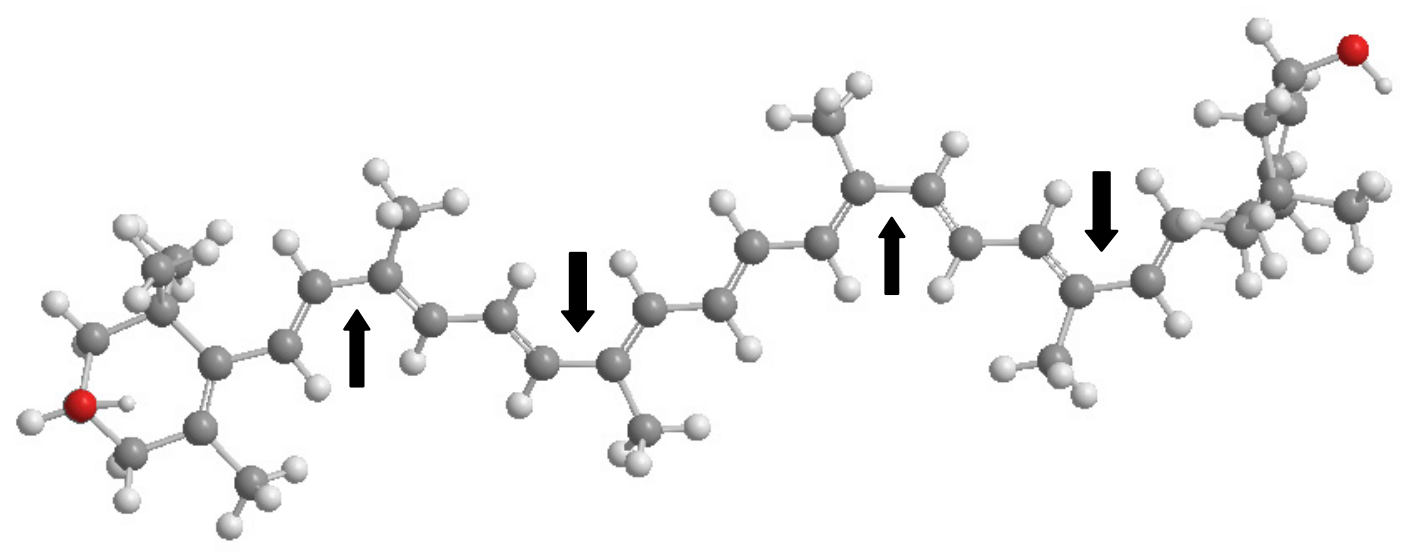

Figure 29: Example: tetra s-cis 2,4,7,9 lutein conformer.

Conformers containing five $s$-cis single bonds

Table 15: Relative energies for conformers containing five $s$-cis single bonds.

\begin{tabular}{|r|r|r|r|r|r|}
\hline $\begin{array}{c}\text { s-cis location/ } \\
\Delta \mathbf{E}(\mathbf{k c a l} / \mathbf{m o l})\end{array}$ & $\mathbf{5}$ & $\mathbf{6}$ & $\mathbf{7}$ & $\mathbf{8}$ & $\mathbf{9}$ \\
\hline $\mathbf{1 2 3 4}$ & 4.1458 & 1.5726 & 0.4266 & 1.8952 & 0.6718 \\
\hline $\mathbf{1 2 3 5}$ & & 5.3192 & 4.0035 & 5.2992 & 4.1063 \\
\hline $\mathbf{1 2 3 6}$ & & & 2.6439 & 4.3776 & 3.0791 \\
\hline $\mathbf{1 2 3 7}$ & & & & 3.2642 & 1.2891 \\
\hline $\mathbf{1 2 3 8}$ & & & & & 2.6867 \\
\hline $\mathbf{1 2 4 5}$ & & 1.726 & 0.5491 & 1.7905 & 0.6148 \\
\hline $\mathbf{1 2 4 6}$ & & & -1.0246 & 0.5048 & -0.8857 \\
\hline $\mathbf{1 2 4 7}$ & & & & -0.9936 & -1.4938 \\
\hline $\mathbf{1 2 4 8}$ & & & & & -1.0038 \\
\hline $\mathbf{1 2 5 6}$ & & & 2.5619 & 3.1239 & 2.7009 \\
\hline
\end{tabular}


Table 15 continues:

\begin{tabular}{|r|r|r|r|r|r|}
\hline & $\mathbf{5}$ & $\mathbf{6}$ & $\mathbf{7}$ & $\mathbf{8}$ & $\mathbf{9}$ \\
\hline $\mathbf{1 2 5 7}$ & & & & 3.4689 & -0.0008 \\
\hline $\mathbf{1 2 5 8}$ & & & & & 1.5665 \\
\hline $\mathbf{1 2 6 7}$ & & & & 1.2552 & -0.3854 \\
\hline $\mathbf{1 2 6 8}$ & & & & & 1.2258 \\
\hline $\mathbf{1 2 7 8}$ & & & & & -0.2397 \\
\hline
\end{tabular}

Table 16: Relative energies for conformers containing five s-cis single bonds.

\begin{tabular}{|r|r|r|r|r|}
\hline $\begin{array}{r}\text { s-cis location/ } \\
\Delta \mathbf{E}(\mathbf{k c a l} / \mathbf{m o l})\end{array}$ & $\mathbf{6}$ & $\mathbf{7}$ & $\mathbf{8}$ & $\mathbf{9}$ \\
\hline $\mathbf{1 3 4 5}$ & 4.745 & 4.2102 & 4.6313 & 4.402 \\
\hline $\mathbf{1 3 4 6}$ & & 1.7095 & 4.8767 & 1.8766 \\
\hline $\mathbf{1 3 4 7}$ & & & 1.6804 & 0.5461 \\
\hline $\mathbf{1 3 4 8}$ & & & & 1.5181 \\
\hline $\mathbf{1 3 5 6}$ & & 5.6351 & 6.6292 & 5.1946 \\
\hline $\mathbf{1 3 5 7}$ & & & 6.2012 & 3.8939 \\
\hline $\mathbf{1 3 5 8}$ & & & & 5.1679 \\
\hline $\mathbf{1 3 6 7}$ & & & 3.9311 & 3.8939 \\
\hline $\mathbf{1 3 6 8}$ & & & & 4.5984 \\
\hline $\mathbf{1 3 7 8}$ & & & & 3.0017 \\
\hline $\mathbf{1 4 5 6}$ & & 2.3477 & 3.8456 & 2.4948 \\
\hline $\mathbf{1 4 5 7}$ & & & 3.4478 & 1.2961 \\
\hline $\mathbf{1 4 5 8}$ & & & & 2.5098 \\
\hline $\mathbf{1 4 6 7}$ & & & 1.4401 & -0.3649 \\
\hline $\mathbf{1 4 6 8}$ & & & & 1.2254 \\
\hline $\mathbf{1 4 7 8}$ & & & & -0.2372 \\
\hline $\mathbf{1 5 6 7}$ & & & 4.8533 & 3.3456 \\
\hline $\mathbf{1 5 6 8}$ & & & & 4.8819 \\
\hline $\mathbf{1 6 7 8}$ & & & & 2.1387 \\
\hline & & & & \\
\hline
\end{tabular}

Table 17: Relative energies for conformers containing five $s$-cis single bonds.

\begin{tabular}{|r|r|r|r|r|}
\hline $\begin{array}{r}\text { s-cis location/ } \\
\Delta \mathbf{E}(\mathbf{k c a l} / \mathbf{m o l})\end{array}$ & $\mathbf{6}$ & $\mathbf{7}$ & $\mathbf{8}$ & $\mathbf{9}$ \\
\hline $\mathbf{2 3 4 5}$ & 4.2487 & 3.075 & 4.3159 & 3.2456 \\
\hline $\mathbf{2 3 4 6}$ & & 1.2031 & 2.8189 & 0.4318 \\
\hline $\mathbf{2 3 4 7}$ & & & 1.5336 & 0.3886 \\
\hline $\mathbf{2 3 4 8}$ & & & & 1.6271 \\
\hline
\end{tabular}


Table 17 continues:

\begin{tabular}{|r|r|r|r|r|}
\hline & $\mathbf{6}$ & $\mathbf{7}$ & $\mathbf{8}$ & $\mathbf{9}$ \\
\hline $\mathbf{2 3 5 6}$ & & 5.0534 & 6.6212 & 5.1865 \\
\hline $\mathbf{2 3 5 7}$ & & & 5.2466 & 3.9505 \\
\hline $\mathbf{2 3 5 8}$ & & & & 5.1506 \\
\hline $\mathbf{2 3 6 7}$ & & & 3.5462 & 2.3872 \\
\hline $\mathbf{2 3 6 8}$ & & & & 4.1668 \\
\hline $\mathbf{2 3 7 8}$ & & & & 3.0161 \\
\hline $\mathbf{2 4 5 6}$ & & 1.3459 & 2.968 & 1.5177 \\
\hline $\mathbf{2 4 5 7}$ & & & 1.5518 & 0.3425 \\
\hline $\mathbf{2 4 5 8}$ & & & & 1.4685 \\
\hline $\mathbf{2 4 6 7}$ & & & 0.3582 & -1.4008 \\
\hline $\mathbf{2 4 6 8}$ & & & & 0.2191 \\
\hline $\mathbf{2 4 7 8}$ & & & & -1.2093 \\
\hline $\mathbf{2 5 6 7}$ & & & 5.2902 & 2.3739 \\
\hline $\mathbf{2 5 6 8}$ & & & & 3.9208 \\
\hline $\mathbf{2 5 7 8}$ & & & & 2.4988 \\
\hline $\mathbf{2 6 7 8}$ & & & & 1.1785 \\
\hline
\end{tabular}

Table 18: Relative energies for conformers containing five $s$-cis single bonds.

\begin{tabular}{|r|r|r|r|}
\hline $\begin{array}{r}\text { s-cis location/ } \\
\Delta \mathbf{E}(\mathbf{k c a l} / \mathbf{m o l})\end{array}$ & $\mathbf{7}$ & $\mathbf{8}$ & $\mathbf{9}$ \\
\hline $\mathbf{3 4 5 6}$ & 4.5607 & 6.193 & 4.7207 \\
\hline $\mathbf{3 4 5 7}$ & & 4.9047 & 4.3513 \\
\hline $\mathbf{3 4 5 8}$ & & & 5.4283 \\
\hline $\mathbf{3 4 6 7}$ & & 3.8136 & 1.8258 \\
\hline $\mathbf{3 4 6 8}$ & & & 3.2204 \\
\hline $\mathbf{3 4 7 8}$ & & & 1.9921 \\
\hline $\mathbf{3 5 6 7}$ & & 6.5296 & 5.6974 \\
\hline $\mathbf{3 5 6 8}$ & & & 6.5772 \\
\hline $\mathbf{3 5 7 8}$ & & & 5.611 \\
\hline $\mathbf{3 6 7 8}$ & & & 4.0288 \\
\hline $\mathbf{4 5 6 7}$ & & 3.4767 & 1.943 \\
\hline $\mathbf{4 5 6 8}$ & & & 3.1782 \\
\hline $\mathbf{4 5 7 8}$ & & & 3.1782 \\
\hline $\mathbf{4 6 7 8}$ & & & 1.279 \\
\hline $\mathbf{5 6 7 8}$ & & & 5.0578 \\
\hline
\end{tabular}




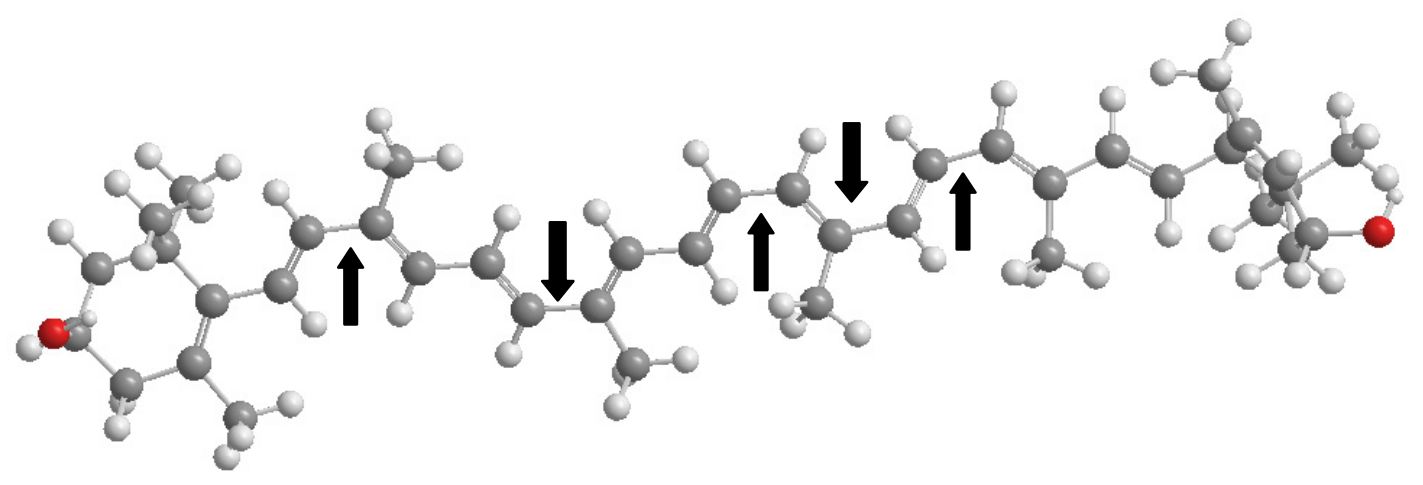

Figure 30: Example: penta s-cis 2,4,6,7,8 lutein conformer.

Conformers containing six $s$-cis single bonds

Table 19: Relative energies for conformers containing six $s$-cis single bonds.

\begin{tabular}{|r|r|r|r|r|}
\hline $\begin{array}{r}\text { s-cis location/ } \\
\Delta \mathbf{E}(\mathbf{k c a l} / \mathbf{m o l})\end{array}$ & $\mathbf{6}$ & $\mathbf{7}$ & $\mathbf{8}$ & $\mathbf{9}$ \\
\hline $\mathbf{1 2 3 4 5}$ & 4.453 & 6.5185 & 4.8319 & 3.9057 \\
\hline $\mathbf{1 2 3 4 6}$ & & 1.2583 & 2.8212 & 1.4489 \\
\hline $\mathbf{1 2 3 4 7}$ & & & 1.4377 & 0.2827 \\
\hline $\mathbf{1 2 3 4 8}$ & & & & 1.6407 \\
\hline $\mathbf{1 2 3 5 6}$ & & 4.9201 & 6.5185 & 5.1402 \\
\hline $\mathbf{1 2 3 5 7}$ & & & 3.861 & 3.8189 \\
\hline $\mathbf{1 2 3 5 8}$ & & & & 5.2608 \\
\hline $\mathbf{1 2 3 6 7}$ & & & 3.4618 & 2.3996 \\
\hline $\mathbf{1 2 3 6 8}$ & & & & 5.1402 \\
\hline $\mathbf{1 2 3 7 8}$ & & & & 2.5247 \\
\hline $\mathbf{1 2 4 5 6}$ & & 1.3826 & 2.9358 & 1.5565 \\
\hline $\mathbf{1 2 4 5 7}$ & & & 2.5375 & 0.3891 \\
\hline $\mathbf{1 2 4 5 8}$ & & & & 1.598 \\
\hline $\mathbf{1 2 4 6 7}$ & & & 0.4022 & -1.219 \\
\hline $\mathbf{1 2 4 6 8}$ & & & & 0.2868 \\
\hline $\mathbf{1 2 4 7 8}$ & & & & -1.0997 \\
\hline $\mathbf{1 2 5 6 7}$ & & & 3.8902 & 2.3513 \\
\hline $\mathbf{1 2 5 6 8}$ & & & & 3.8987 \\
\hline $\mathbf{1 2 5 7 8}$ & & & & 3.203 \\
\hline $\mathbf{1 2 6 7 8}$ & & & & 1.1188 \\
\hline & & & & \\
\hline & & & & \\
\hline & & & & \\
\hline & & & & \\
\hline & & & & \\
\hline & & & & \\
\hline
\end{tabular}


Table 20: Relative energies for conformers containing six s-cis single bonds.

\begin{tabular}{|r|r|r|r|}
\hline $\begin{array}{r}\text { s-cis location/ } \\
\Delta \mathbf{E}(\mathbf{k c a l} / \mathbf{m o l})\end{array}$ & $\mathbf{7}$ & $\mathbf{8}$ & $\mathbf{9}$ \\
\hline $\mathbf{1 3 4 5 6}$ & 4.4828 & 5.9184 & 4.4569 \\
\hline $\mathbf{1 3 4 5 7}$ & & 4.2959 & 1.2269 \\
\hline $\mathbf{1 3 4 5 8}$ & & & 5.1771 \\
\hline $\mathbf{1 3 4 6 7}$ & & 3.1152 & 1.6005 \\
\hline $\mathbf{1 3 4 6 8}$ & & & 3.2506 \\
\hline $\mathbf{1 3 4 7 8}$ & & & 1.5135 \\
\hline $\mathbf{1 3 5 6 7}$ & & 6.2477 & 5.6817 \\
\hline $\mathbf{1 3 5 6 8}$ & & & 6.9354 \\
\hline $\mathbf{1 3 5 7 8}$ & & & 4.9041 \\
\hline $\mathbf{1 3 6 7 8}$ & & & 3.8372 \\
\hline $\mathbf{1 4 5 6 7}$ & & 3.6349 & 2.1273 \\
\hline $\mathbf{1 4 5 6 8}$ & & & 3.6564 \\
\hline $\mathbf{1 4 5 7 8}$ & & & 3.3434 \\
\hline $\mathbf{1 4 6 7 8}$ & & & 1.4457 \\
\hline $\mathbf{1 5 6 7 8}$ & & & 4.9115 \\
\hline & & & \\
\hline
\end{tabular}

Table 21: Relative energies for conformers containing six s-cis single bonds.

\begin{tabular}{|r|r|r|r|}
\hline $\begin{array}{r}\text { s-cis location/ } \\
\Delta \mathbf{E}(\mathbf{k c a l} / \mathbf{m o l})\end{array}$ & $\mathbf{7}$ & $\mathbf{8}$ & $\mathbf{9}$ \\
\hline $\mathbf{2 3 4 5 6}$ & 3.8312 & 4.9189 & 4.0351 \\
\hline $\mathbf{2 3 4 5 7}$ & & 3.9544 & 2.887 \\
\hline $\mathbf{2 3 4 5 8}$ & & & 4.0788 \\
\hline $\mathbf{2 3 4 6 7}$ & & 3.8851 & 1.0096 \\
\hline $\mathbf{2 3 4 6 8}$ & & & 2.5499 \\
\hline $\mathbf{2 3 4 7 8}$ & & & 1.3016 \\
\hline $\mathbf{2 3 5 6 7}$ & & 6.556 & 4.8591 \\
\hline $\mathbf{2 3 5 6 8}$ & & & 6.3913 \\
\hline $\mathbf{2 3 5 7 8}$ & & & 5.0023 \\
\hline $\mathbf{2 3 6 7 8}$ & & & 3.2613 \\
\hline $\mathbf{2 4 5 6 7}$ & & 3.0456 & 1.1578 \\
\hline $\mathbf{2 4 5 6 8}$ & & & 2.7271 \\
\hline $\mathbf{2 4 5 7 8}$ & & & 1.3186 \\
\hline $\mathbf{2 4 6 7 8}$ & & & 0.0425 \\
\hline $\mathbf{2 5 6 7 8}$ & & & 4.0707 \\
\hline & & & \\
\hline
\end{tabular}


Table 22: Relative energies for conformers containing six s-cis single bonds.

\begin{tabular}{|r|r|r|}
\hline $\begin{array}{l}\text { s-cis location/ } \\
\Delta \mathbf{E}(\text { kcal/mol) }\end{array}$ & $\mathbf{8}$ & $\mathbf{9}$ \\
\hline $\mathbf{3 4 5 6 7}$ & 7.1866 & 4.3861 \\
\hline $\mathbf{3 4 5 6 8}$ & & 5.8982 \\
\hline $\mathbf{3 4 5 7 8}$ & & 4.6781 \\
\hline $\mathbf{3 4 6 7 8}$ & & 3.3729 \\
\hline $\mathbf{3 5 6 7 8}$ & & 6.2676 \\
\hline $\mathbf{4 5 6 7 8}$ & & 3.0356 \\
\hline
\end{tabular}

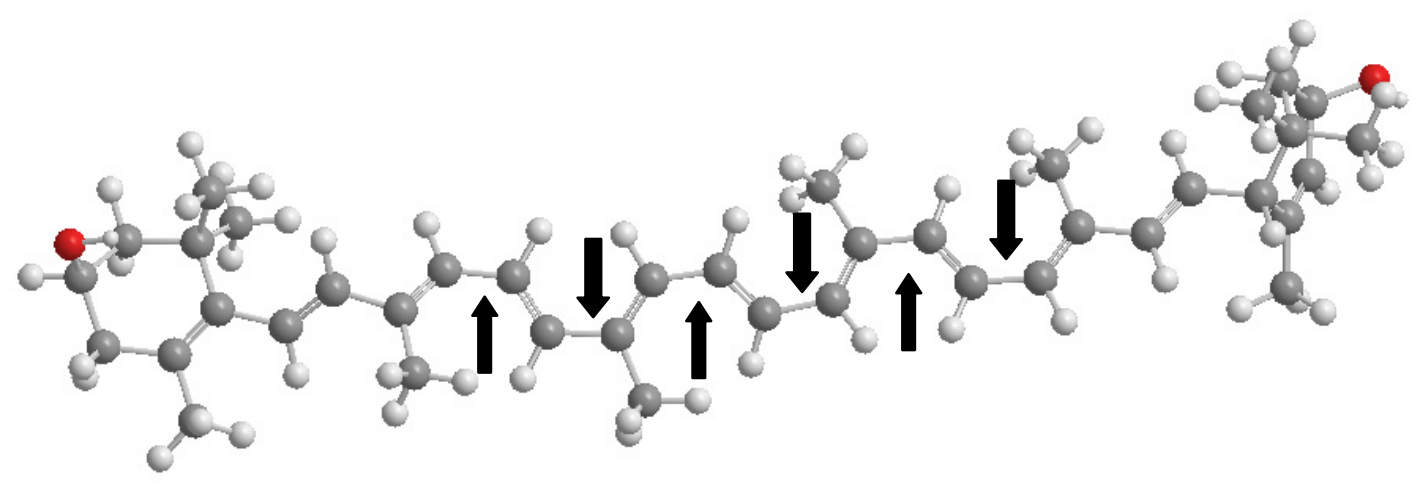

Figure 31: Example: hexa s-cis 3,4,5,6,7,8 lutein conformer.

Conformers containing seven s-cis single bonds

Table 23: Relative energies for conformers containing seven s-cis single bonds.

\begin{tabular}{|r|r|r|r|}
\hline $\begin{array}{l}\text { s-cis location/ } \\
\Delta \mathbf{E} \text { (kcal/mol) }\end{array}$ & $\mathbf{7}$ & $\mathbf{8}$ & $\mathbf{9}$ \\
\hline $\mathbf{1 2 3 4 5 6}$ & 4.1748 & 5.3929 & 4.0519 \\
\hline $\mathbf{1 2 3 4 5 7}$ & & 5.2741 & 3.133 \\
\hline $\mathbf{1 2 3 4 5 8}$ & & & 4.5018 \\
\hline $\mathbf{1 2 3 4 6 7}$ & & 2.6269 & 1.0052 \\
\hline $\mathbf{1 2 3 4 6 8}$ & & & 2.5759 \\
\hline $\mathbf{1 2 3 4 7 8}$ & & & 1.2664 \\
\hline $\mathbf{1 2 3 5 6 7}$ & & 6.4002 & 4.7895 \\
\hline $\mathbf{1 2 3 5 6 8}$ & & & 6.3039 \\
\hline $\mathbf{1 2 3 5 7 8}$ & & & 5.5254 \\
\hline $\mathbf{1 2 3 6 7 8}$ & & & 3.2605 \\
\hline $\mathbf{1 2 4 5 6 7}$ & & 2.6986 & 1.1635 \\
\hline
\end{tabular}


Table 23 continues:

\begin{tabular}{|r|r|r|r|}
\hline & $\mathbf{7}$ & $\mathbf{8}$ & $\mathbf{9}$ \\
\hline $\mathbf{1 2 4 5 6 8}$ & & & 2.6661 \\
\hline $\mathbf{1 2 4 5 7 8}$ & & & 2.3972 \\
\hline $\mathbf{1 2 4 6 7 8}$ & & & 0.4631 \\
\hline $\mathbf{1 2 5 6 7 8}$ & & & 3.957 \\
\hline $\mathbf{1 3 4 5 6 7}$ & & 5.4869 & 3.8966 \\
\hline $\mathbf{1 3 4 5 6 8}$ & & & 5.7017 \\
\hline $\mathbf{1 3 4 5 7 8}$ & & & 5.7188 \\
\hline $\mathbf{1 3 4 6 7 8}$ & & & 2.8482 \\
\hline $\mathbf{1 3 5 6 7 8}$ & & & 6.7334 \\
\hline $\mathbf{1 4 5 6 7 8}$ & & & 3.6977 \\
\hline
\end{tabular}

Table 24 Relative energies for conformers containing seven s-cis single bonds.

\begin{tabular}{|r|r|r|}
\hline $\begin{array}{c}\text { s-cis location/ } \\
\Delta \mathbf{E}(\mathbf{k c a l} / \mathbf{m o l})\end{array}$ & $\mathbf{8}$ & $\mathbf{9}$ \\
\hline $\mathbf{2 3 4 5 6 7}$ & 5.1413 & 3.6442 \\
\hline $\mathbf{2 3 4 5 6 8}$ & & 4.6401 \\
\hline $\mathbf{2 3 4 5 7 8}$ & & 3.735 \\
\hline $\mathbf{2 3 4 6 7 8}$ & & 3.735 \\
\hline $\mathbf{2 3 5 6 7 8}$ & & 6.2518 \\
\hline $\mathbf{2 4 5 6 7 8}$ & & 2.6293 \\
\hline $\mathbf{3 4 5 6 7 8}$ & & 5.9604 \\
\hline
\end{tabular}

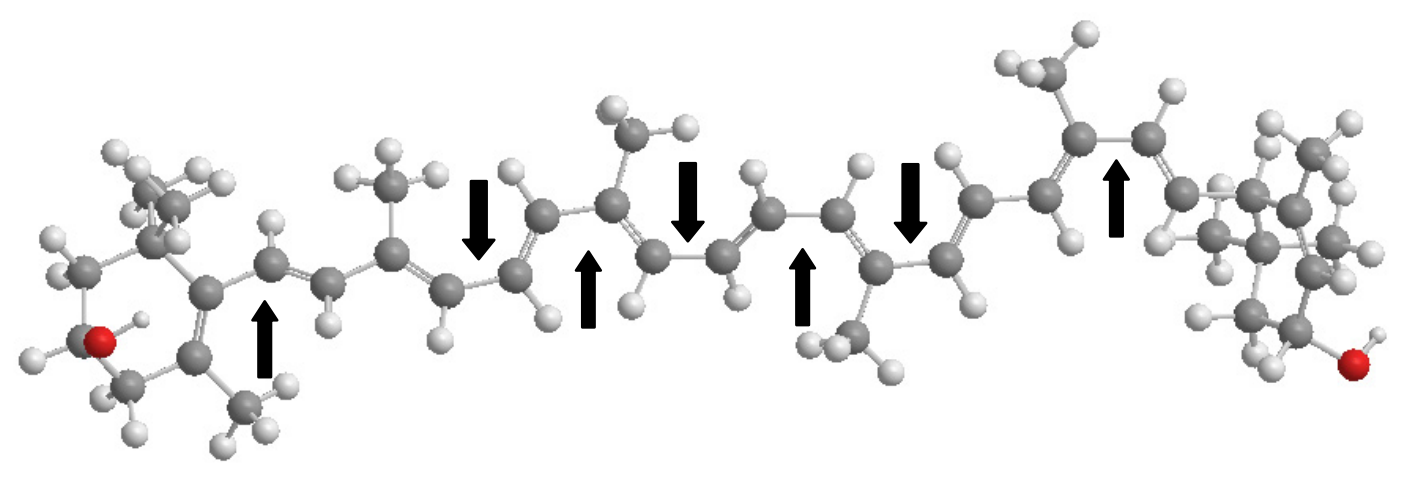

Figure 32: Example: hepta s-cis 1,3,4,5,6,7,9 lutein conformer. 
Conformers containing eight $s$-cis single bonds

Table 25 Relative energies for conformers containing eight $s$-cis single bonds.

\begin{tabular}{|c|r|r|r|r|r|}
\hline $\begin{array}{c}\text { s-cis } \\
\text { location }\end{array}$ & $\mathbf{1 2 3 4 5 6 7 8}$ & $\mathbf{1 2 3 4 5 6 7 9}$ & $\mathbf{1 2 3 4 5 6 8 9}$ & $\mathbf{1 2 3 4 5 7 8 9}$ & $\mathbf{1 2 3 4 6 7 8 9}$ \\
\hline $\begin{array}{c}\Delta \text { Energie } \\
\text { (kcal/mol) }\end{array}$ & 5.0392 & 3.7859 & 5.2398 & 5.3723 & 2.5761 \\
\hline
\end{tabular}

Table 26 Relative energies for conformers containing eight $s$-cis single bonds.

\begin{tabular}{|c|r|r|r|r|}
\hline s-cis location & $\mathbf{1 2 3 5 6 7 8 9}$ & $\mathbf{1 2 4 5 6 7 8 9}$ & $\mathbf{1 3 4 5 6 7 8 9}$ & $\mathbf{2 3 4 5 6 7 8 9}$ \\
\hline & & & & \\
\hline Energie (kcal/mol) & 6.3434 & 2.7517 & 5.6877 & 4.8792 \\
\hline
\end{tabular}

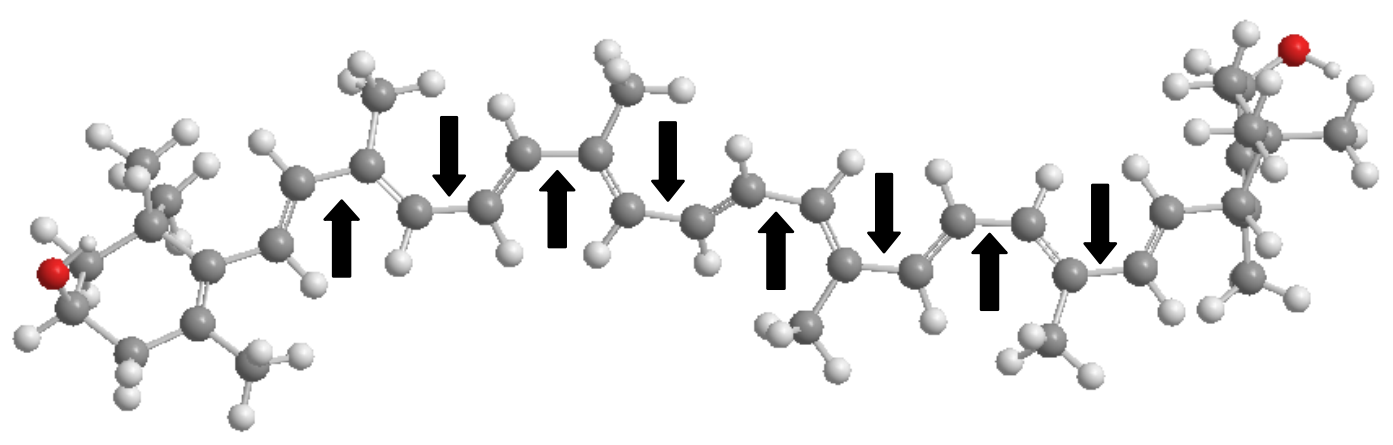

Figure 33: Example: octa s-cis 2,3,4,5,6,7,8,9 lutein conformer.

Conformer containing nine $s$-cis single bond

Table 27 Relative energies for the conformer with nine s-cis single bonds.

\begin{tabular}{|c|r|}
\hline s-cis location & $\mathbf{1 2 3 4 5 6 7 8 9}$ \\
\hline$\Delta$ Energy (kcal/mol) & 5.2324 \\
\hline
\end{tabular}




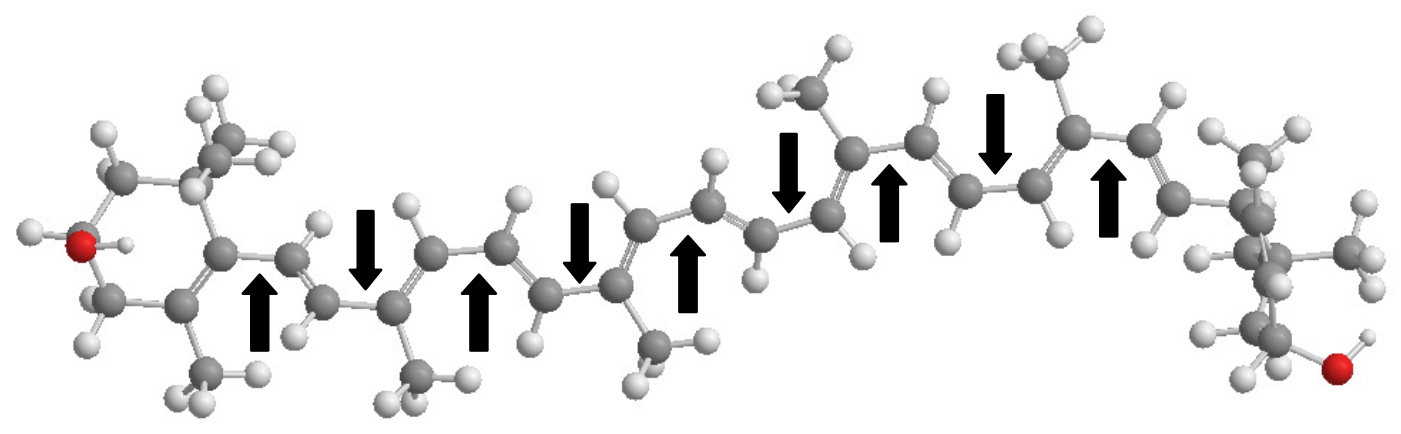

Figure 34: Example: nona s-cis 1,2,3,4,5,6,7,8,9 lutein conformer.

\subsubsection{Calculations for the complete carotenoid model using B3* LYP/6-31G*.}

From among all the conformers that the model can have we selected some of the conformers to compare the calculations run with the semi-empirical PM3 method and the B3*LYP method. The results are shown is Table 28.

Table 28: Calculations with B $3 *$ LYP for selected conformers of the lutein model.

\begin{tabular}{|c|c|r|}
\hline Conformer & $\mathbf{E}(\mathbf{k c a l} / \mathbf{m o l})$ & \multicolumn{1}{c|}{$\Delta \mathbf{E}$} \\
\hline lutein & -1073692.21 & 0.00 \\
\hline all-E lutein & -1073689.81 & 2.39 \\
\hline $\mathbf{2}$ & -1073687.09 & 5.12 \\
\hline $\mathbf{3}$ & -1073685.34 & 6.87 \\
\hline $\mathbf{4}$ & -1073687.43 & 4.78 \\
\hline $\mathbf{5}$ & -1073685.93 & 6.28 \\
\hline $\mathbf{6}$ & -1073685.89 & 6.32 \\
\hline $\mathbf{7}$ & -1073687.26 & 4.95 \\
\hline $\mathbf{8}$ & -1073685.48 & 6.73 \\
\hline $\mathbf{9}$ & -1073687.25 & 5.15 \\
\hline $\mathbf{2 4}$ & -1073684.05 & 8.16 \\
\hline $\mathbf{2 6}$ & -1073682.80 & 9.41 \\
\hline $\mathbf{2 7}$ & -1073684.04 & 8.15 \\
\hline $\mathbf{3 5}$ & -1073681.17 & 11.04 \\
\hline $\mathbf{4 9}$ & -1073683.60 & 7.92 \\
\hline
\end{tabular}




\begin{tabular}{|c|c|c|}
\hline $\mathbf{5 6}$ & -1073680.69 & 11.51 \\
\hline $\mathbf{6 8}$ & -1073681.32 & 10.89 \\
\hline $\mathbf{2 4 7}$ & -1073680.67 & 11.54 \\
\hline $\mathbf{2 4 8}$ & -1073679.40 & 12.81 \\
\hline $\mathbf{2 4 9}$ & -1073680.88 & 11.33 \\
\hline $\mathbf{3 6 8}$ & -1073676.47 & 15.74 \\
\hline $\mathbf{3 7 9}$ & -1073679.44 & 12.77 \\
\hline $\mathbf{4 7 9}$ & -1073679.99 & 11.35 \\
\hline $\mathbf{4 8 9}$ & -1073680.03 & 12.18 \\
\hline $\mathbf{1 2 6 9}$ & -1073681.47 & 10.74 \\
\hline $\mathbf{2 4 7 9}$ & -1073677.43 & 14.78 \\
\hline $\mathbf{3 5 6 8}$ & -1073670.95 & 21.26 \\
\hline $\mathbf{4 6 7 8}$ & -1073675.00 & 17.20 \\
\hline $\mathbf{1 3 4 8 9}$ & -1073677.36 & 14.84 \\
\hline $\mathbf{2 3 4 5 6}$ & -1073670.97 & 21.24 \\
\hline $\mathbf{2 4 6 7 8}$ & -1073671.53 & 20.68 \\
\hline $\mathbf{2 3 4 5 6 8}$ & -1073665.08 & 27.12 \\
\hline $\mathbf{3 4 5 6 7 8}$ & -1073666.39 & 25.82 \\
\hline $\mathbf{3 5 6 7 8 9}$ & -1073665.14 & 27.07 \\
\hline $\mathbf{4 5 6 7 8 9}$ & -1073669.12 & 23.08 \\
\hline $\mathbf{2 3 5 6 7 8 9}$ & -1073662.61 & 29.60 \\
\hline $\mathbf{1 2 3 4 5 6 7 8 9}$ & -1708.26709 & 29.60 \\
\hline & & \\
\hline
\end{tabular}


2.3.4. Calculations for the rotational barrier of the single bonds in lutein model using the B3*LYP method

Calculations to find the rotational barrier for the $4^{\text {th }}(\mathrm{C} 12-\mathrm{C} 13)$ and $5^{\text {th }}(\mathrm{C} 14-\mathrm{C} 15)$ bond were run using the B3*LYP method. Table 29 shows the results for each case and Figure 35 shows the torsional potential for the bonds.

Table 29: Calculations for dihedral angle for the $4^{\text {th }}$ and $5^{\text {th }}$ single bond in the lutein model.

\begin{tabular}{|c|c|r|c|r|}
\cline { 2 - 5 } \multicolumn{1}{c|}{} & \multicolumn{2}{c|}{ 4th single bond C12-C13 } & \multicolumn{2}{c|}{ 5th single bond C14-C15 } \\
\hline dihedral angle & $\mathbf{E}(\mathbf{k c a l} / \mathbf{m o l})$ & \multicolumn{1}{c|}{$\Delta \mathbf{E}$} & $\mathbf{E}(\mathbf{k c a l} / \mathbf{m o l})$ & \multicolumn{1}{c|}{$\Delta \mathbf{E}$} \\
\hline lutein trans $(180)$ & -1073690.89 & 0 & -1073690.90 & 0 \\
\hline 150 & -1073689.51 & 1.38 & -1073689.88 & 1.02 \\
\hline 120 & -1073683.90 & 6.99 & -1073684.41 & 6.49 \\
\hline 90 & -1073681.55 & 9.35 & -1073681.16 & 9.74 \\
\hline 60 & -1073685.34 & 5.55 & -1073684.07 & 6.83 \\
\hline 30 & -1073688.42 & 2.47 & -1073687.04 & 3.86 \\
\hline 0 & -1073688.70 & 2.19 & -1073687.20 & 3.70 \\
\hline
\end{tabular}

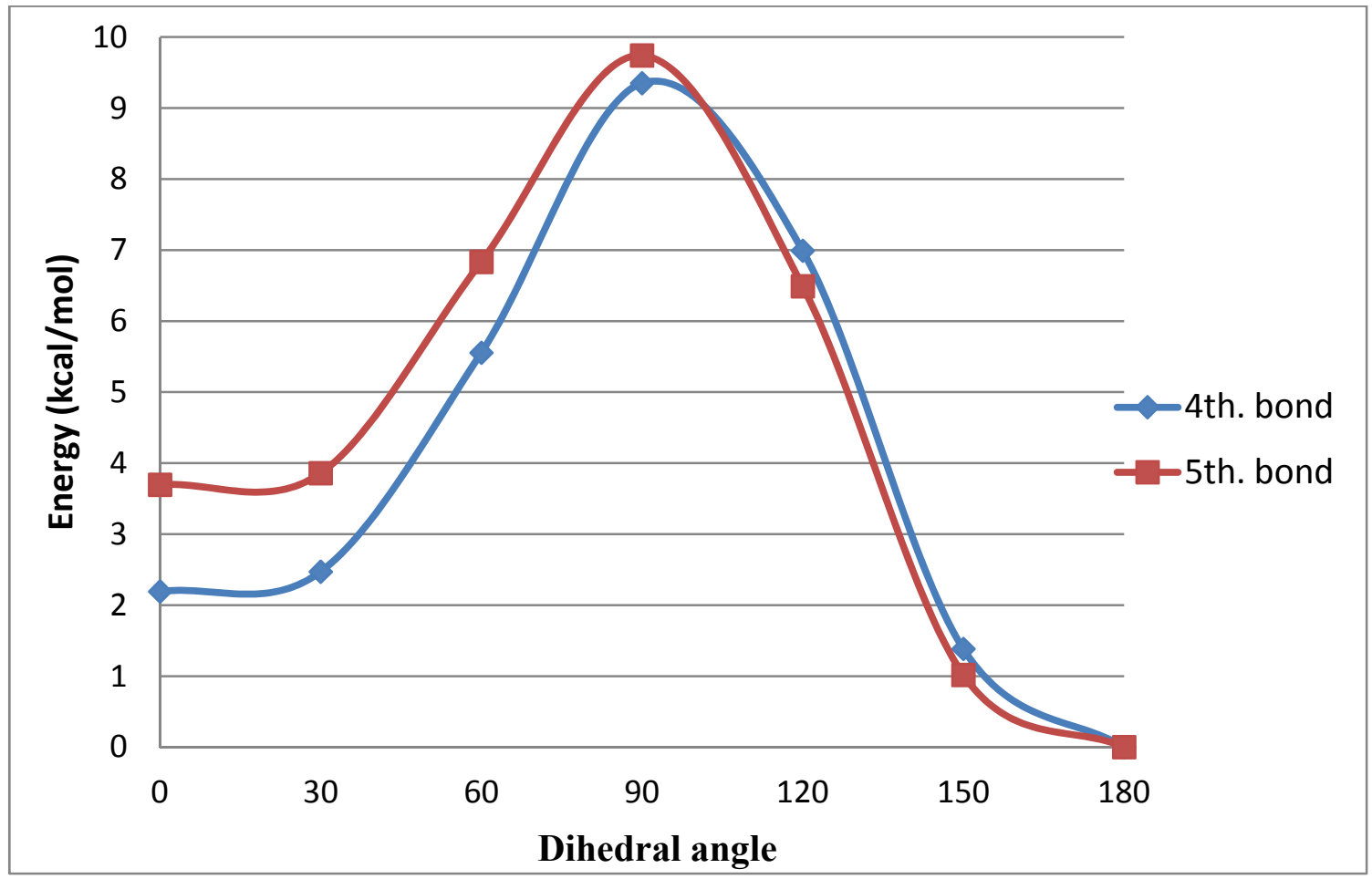

Figure 35: Energy barrier for the rotation of the single bond at the $4^{\text {th }}$ and $5^{\text {th }}$ bond in the lutein model. 


\subsection{Discussion}

Below, Figure 36 maps the energy of the lutein conformers according to the numbers of s-cis bonds present. From the figure, we can see that the distribution of the energies of the lutein conformers lay mostly between 0 and $4 \mathrm{kcal} / \mathrm{mol}$.

\begin{tabular}{|c|c|c|c|c|c|c|c|c|c|}
\hline $\begin{array}{c}\text { \#s-cis/ } \\
\mathrm{kcal} / \mathrm{mol}\end{array}$ & 1 & 2 & 3 & 4 & 5 & 6 & 7 & 8 & 9 \\
\hline $0-1$ & & & & & & & & & \\
\hline $1-2$ & & & & & & & & & \\
\hline $2-3$ & & & & & & & & & \\
\hline $3-4$ & & & & & & & & & \\
\hline $4-5$ & & & & & & & & & \\
\hline $5-6$ & & & & & & & & & \\
\hline $6-7$ & & & & & & & & & \\
\hline $7-8$ & & & & & & & & & \\
\hline
\end{tabular}

\begin{tabular}{|l|l|l|l|l|l|l|l|l|}
\hline \# conformers & $1-4$ & $4-8$ & $9-12$ & $13-17$ & $18-22$ & $23-27$ & $28-31$ & $31-35$ \\
\hline & & & & & & & & \\
\hline
\end{tabular}

Figure 36: The energy of the lutein conformers according to the number of $s$-cis bonds and their location within the molecule.

A surprising observation is that the conformer containing $9 s$-cis bonds is lower in energy than some others having 3 or $6 s$-cis bonds in their structures. The conformer with the highest energy according to the PM3 method is the tetra s-cis 3568 lutein conformer and not the nona $s$ cis 123456789 lutein conformer, as we might have predicted. Another result observed about the conformers is that the position and arrangement of the methyl groups affects the stability of the different conformers. In lutein we can separate s-single bonds into two groups. In the first group, methyl substitution at single bonds $2,4,7$, and 9 reduces the energy differences between the s-cis and s-trans conformers. In contrast the second group of single bonds, those labeled 3, 5, 6, and 8 lack a methyl resulting in a more dramatic difference in the energies of the two conformers 
because the alkyl chains are oriented away from each other in the $s$-trans conformation. Bonds 2 , 4, 7, and 9 are analogs to 2-methyl-1,3-butadiene (see Figure 21.b) and 3, 5, 6, and 8 (see Figure 21.c) are analogs to 1,3-pentadiene. If we take 1,3-butadiene as a reference we can say that the substitution at the second carbon lowers the energy for the isomerism cis-trans compared to substitution of a methyl group at the first carbon (1,3 pentadiene). The energies for the cis-trans conformers of the substituted 1,3-butadiene (Table 3) show that there is an approximate 1 $\mathrm{kcal} / \mathrm{mol}$ difference between them. Conformers with the s-cis bonds in the first group of single bonds have lower energy.

Table 30: Correlation of $\Delta \mathrm{E}(\mathrm{kcal} / \mathrm{mol})$ values between $s$-cis and $s$-trans conformers as calculated using PM3 and B3LYP/6-31G*.

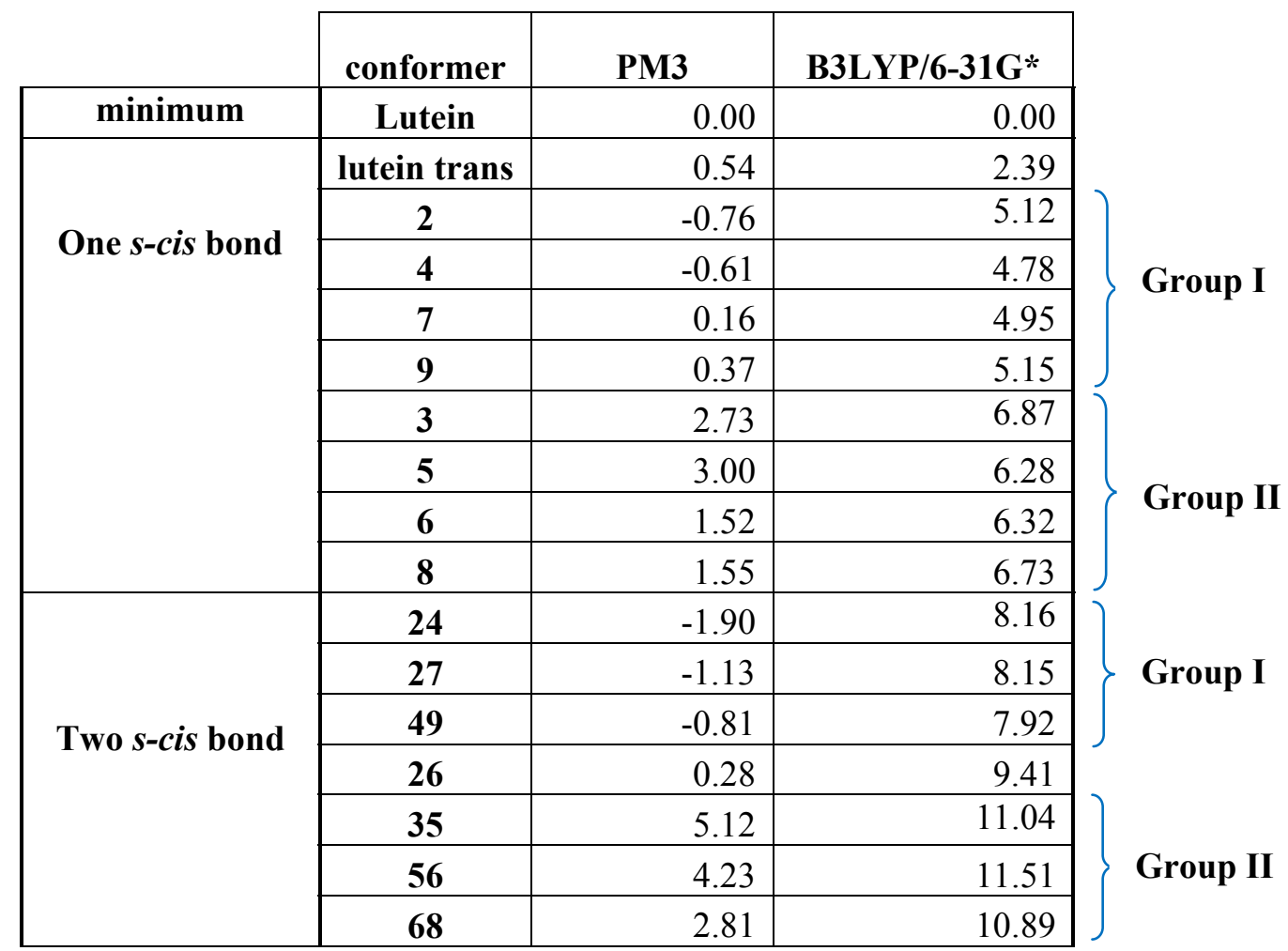




\begin{tabular}{|c|c|r|r|}
\hline \multirow{4}{*}{$\begin{array}{c}\text { Three } s \text {-cis } \\
\text { bonds }\end{array}$} & $\mathbf{2 4 7}$ & -2.24 & 11.54 \\
\cline { 2 - 4 } & $\mathbf{2 4 9}$ & -2.06 & 11.33 \\
\cline { 2 - 4 } & $\mathbf{4 7 9}$ & -1.15 & 11.35 \\
\cline { 2 - 4 } & $\mathbf{2 4 8}$ & -0.68 & 12.81 \\
\cline { 2 - 4 } & $\mathbf{3 7 9}$ & 2.32 & 12.77 \\
\cline { 2 - 4 } & $\mathbf{4 8 9}$ & 0.25 & 12.18 \\
\cline { 2 - 4 } & $\mathbf{3 6 8}$ & 4.89 & 15.74 \\
\hline \multirow{3}{*}{ Four s-cis bonds } & $\mathbf{1 2 6 9}$ & 0.00 & 10.74 \\
\cline { 2 - 4 } & $\mathbf{2 4 7 9}$ & -2.43 & 14.78 \\
\cline { 2 - 4 } & $\mathbf{3 5 6 8}$ & 7.37 & 21.26 \\
\cline { 2 - 4 } & $\mathbf{4 6 7 8}$ & 1.61 & 17.20 \\
\hline Five s-cis bonds & $\mathbf{1 3 4 8 9}$ & 1.52 & 14.84 \\
\cline { 2 - 4 } & $\mathbf{2 3 4 5 6}$ & 4.25 & 21.24 \\
\cline { 2 - 4 } & $\mathbf{2 4 6 7 8}$ & 0.36 & 20.68 \\
\hline \multirow{3}{*}{ Six s-cis bonds } & $\mathbf{2 3 4 5 6 8}$ & 4.92 & 27.12 \\
\cline { 2 - 4 } & $\mathbf{3 4 5 6 7 8}$ & 7.19 & 25.82 \\
\cline { 2 - 4 } & $\mathbf{3 5 6 7 8 9}$ & 6.27 & 27.07 \\
\cline { 2 - 4 } & $\mathbf{4 5 6 7 8 9}$ & 3.04 & 23.08 \\
\hline Seven $s$-cis bond & $\mathbf{2 3 5 6 7 8 9}$ & 6.25 & 29.60 \\
\hline All s-cis & $\mathbf{1 2 3 4 5 6 7 8 9}$ & 5.23 & 29.60 \\
\hline
\end{tabular}

Table 30 compares the $\Delta \mathrm{E}$ values obtained using PM3 and B3LYP/6-31G* theories. When we compare the values using both methods, we notice that the values for and B3LYP are larger. But the tendency when we subdivide them is similar. In the first group of conformers, those with one $s$-cis bond (Table 30 have a lower relative energy than those in group II. We can see that this tendency repeats for all of the conformers in Table 30. The relative energy of the conformers that possess s-cis bonds that correspond to both group I and group II are intermediate in value compared to the lowest and the highest relative energies. Using the values from Table 30, we observed a good correlation allows use of the PM3 values to predict the B3LYP/6-31G* relative energy for each lutein conformer. The PM3 relative energy corresponds to the variable $\mathrm{m}$ and the number of s-cis bonds is represented by $\mathrm{n}$ in the conformer in the equation below:

\section{B3LYP Energy $=4.14 n+m$}




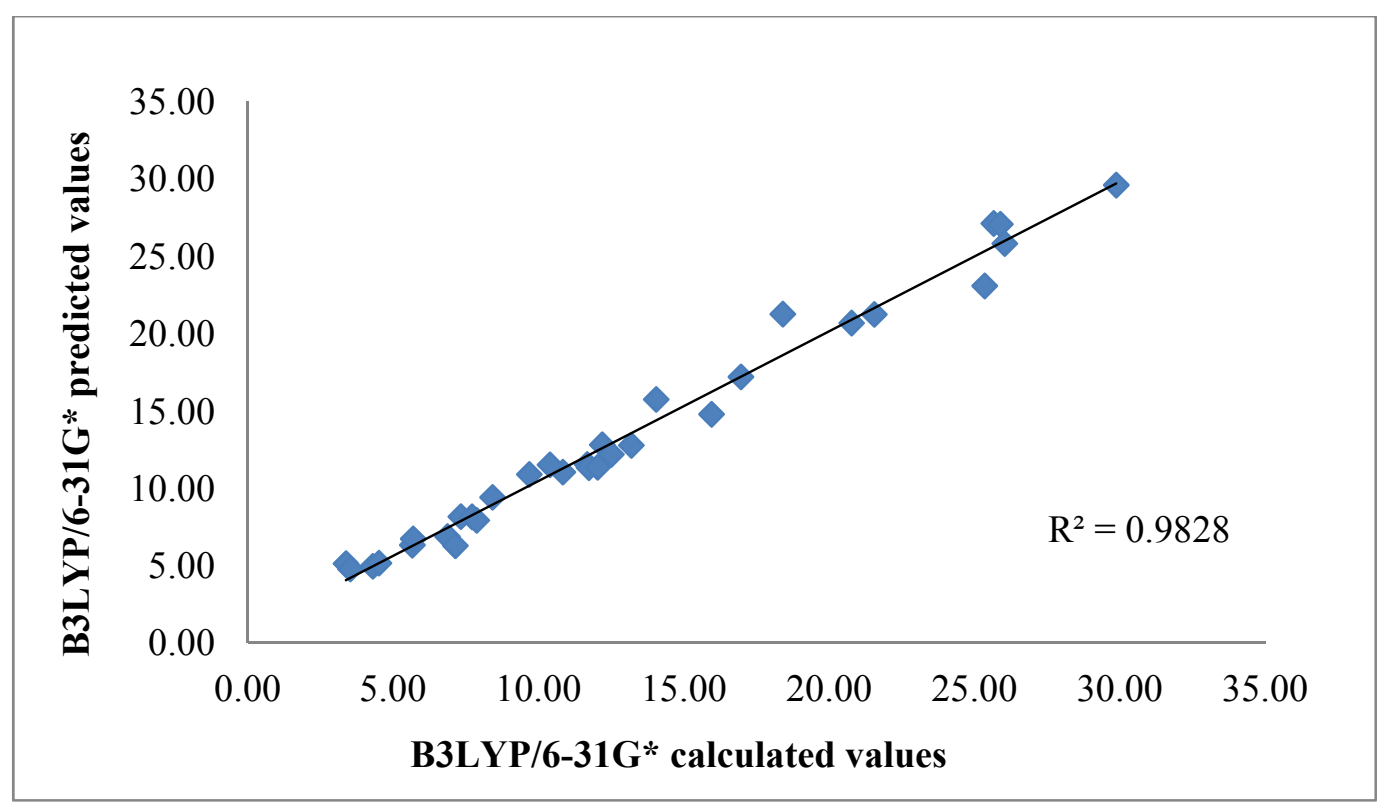

Figure 37: Correlation between the calculated B3LYP/6-31G* DE values and the predicted values using the formula.

Figure 37 shows the correlation of the predicted values based on PM3 and the actual B3LYP results. The values for PM3 are not as precise as the values that are obtained using the density functional theory. When using the B3LYP/6-31G* theory, the values of energy in $\mathrm{kcal} / \mathrm{mol}$ are higher than those obtained by the PM3 method. The square of the correlation coefficient $\left(\mathrm{R}^{2}=0.9828\right)$ in the graph demonstrates that quality of the formula in estimating the computed B3LYP value. The range of relative energies calculated is from 2 to $30 \mathrm{kcal} / \mathrm{mol}$ for the different conformers using the $\mathrm{B} 3 \mathrm{LYP} / 6-31 \mathrm{G}^{*}$ theory. The most accessible conformers are those that fall below $10 \mathrm{kcal} / \mathrm{mol}$. We know that other molecules in nature readily overcome barriers of this magnitude and equilibration of conformations is rapid.. Cyclohexane is an example. The highest energy cyclohexane conformers, the twist chair, are $10.7 \mathrm{kcal} / \mathrm{mol}$ higher than the more preferred chair geometry. In the case of the lutein conformers, the mono and di-cis, conformers are below $10 \mathrm{kcal} / \mathrm{mol}$ in energy. Therefore, these conformers can co- exist although the higher 
energy s-cis will be present in relatively small percentages by comparison to the preferred lutein conformer. The conformers with three s-cis bonds are in a range of 10 to $15 \mathrm{kcal} / \mathrm{mol}$. The rest of the conformers have higher values. Although, it is interesting to note that the tetra s-cis 1269 has energy of only $10.74 \mathrm{kcal} / \mathrm{mol}$. When we focus our attention on those conformers that have the scis single bonds in the group one (i.e. methyls at positions: 2, 4, 7, and 9), as we mentioned before, we can see that the tendency is to have a lower energies in comparison with those in the second group (i.e. methyls at positions: 3, 5, 6, and 8). This explains why the tetra s-cis 1269 lutein conformer has such a low energy relative to the most stable lutein conformer.

Figure 35 shows the rotational barriers that must be overcome for the s-cis single bonds to undergo interconversion to different conformations. The s-cis bonds analyzed are present at $4^{\text {th }}$ and $5^{\text {th }}$ position in the lutein model. As we mentioned before, the first one, shown in blue $\left(4^{\text {th }}\right.$ position) is the analogue of 2-methyl 1,3-butadiene. Calculations made for the rotational barrier of this molecule [46] using ab initio quantum method show a maximum for the gauche conformation of $5.6 \mathrm{kcal} / \mathrm{mol}$ in the vicinity of $90-100^{\circ}$; and the minimum of $2.3 \mathrm{kcal} / \mathrm{mol}$ around $45^{\circ}$ from the syn conformation (s-cis). The second one at the $5^{\text {th }}$ position is shown in red and the rotational barrier for its analogue, 1,3 pentadiene was calculated using a $a b$ initio theory at a MP2/6-31G* level [38]. The maximum energy is $5.9 \mathrm{kcal} / \mathrm{mol}$ for the gauche conformation at $100^{\circ}$ and the minimum energy is $2.6 \mathrm{kcal} / \mathrm{mol}$ at $40^{\circ}$. For both simple models the curves are similar, as is the case for the two s-cis single bonds analyzed in this study. Although, the energy values of the carotenoids are higher when compared to the simple small molecule models, both of the curves have a similar pattern. The maximum energy in both cases was determined to be at $90^{\circ}$ and the difference between them is about $0.39 \mathrm{kcal} / \mathrm{mol}$. The higher relative energies can be attributed to the steric repulsions between the methyl groups in the long lutein model. 
According to the results from the study conducted here, we propose that some of the s-cis conformers, that are sufficiently low in energy, exist in nature. Lutein participates in cellular activities and interacts with proteins and enzymes in the living systems. As a result of these types of interactions at a cellular level, lutein may adapt and adjust to that biological environment by dynamically deforming between a number of different low energy conformers. The significance of this work is that we may expect carotenoids to be stabilized in different conformations by interaction with biological molecules. These different conformations can be expected contribute to the physical and chemical properties as well as the behavior of the carotenoids within the biological environment. Binding of the carotenoid to a protein might readily modulate such important properties as excited state energies by favoring an s-cis conformer in preference to the native s-trans conformations. 


\section{CHAPTER 3: MEASUREMENT OF CAROTENOID LEVELS IN HUMAN SERUM}

\subsection{Overview:}

Special attention has been paid to carotenoids including: lycopene, $\alpha$ - and $\beta$ - carotenes for their response to minimize the risk of cancer and cardiovascular diseases. For the past two decades, many study trials [28, 47-49] in lutein supplementation among healthy subjects and patients with age-related macular disease in early and advanced stages have been carried out to investigate the response of lutein supplementation. Amounts of free lutein given to the subjects vary from $10-30 \mathrm{mg} /$ day and the studies vary in short-term ( 3 months) to up to 4 years longterm periods $[30,50]$. In all the cases, the relationship between the macular pigment caotenoids and AMD has been consistent and therefore, it has been suggested that the risk of Age-related macular degeneration can be reduced by increasing the intake of lutein. Increases in the amount of macular pigment located in the human retina protect these tissues from damage by photoxidation. In addition, the effect of multinutrients (including carotenoids) has been studied in HIV patients [51, 52]. Consequently, lutein supplementation studies are important to the nutritional field. The study of carotenoid levels in human serum is well documented and different methods have been published [53-55]. The dietary intake of lutein in the Western human diet is low $[56,57]$.

In this chapter, we studied six major carotenoids in human serum of subjects that participated in two lutein supplementation studies. We measured the carotenoid levels of lutein (L), zeaxanthin (Z), lycopene (lyc), $\beta$-carotene $(\beta-\mathrm{C}), \alpha$-carotene $(\alpha-\mathrm{C}), \beta$-cryptoxanthin $(\beta$-cry), and $\alpha$-cryptoxanthin ( $\alpha$-cry) and determined the ratios both before and during supplementation. In addition, the macular pigment optical density (MPOD) of the subjects was measured by heterochromatic flicker photometry. The results are compared to determine the effect of the supplementation on elevating the MPOD of the subjects. 
Table 31: Uv-vis data for carotenoids dissolved in ethanol [58] .

\begin{tabular}{|c|ccc|}
\hline Carotenoid & $\lambda_{\max }(\mathbf{n m})$ \\
\hline Lutein & 422 & 445 & 474 \\
\hline Zeaxanthin & 428 & 450 & 478 \\
\hline$\alpha-$ cryptoxanthin & 423 & 446 & 473 \\
\hline$\beta-$ cryptoxanthin & 428 & 450 & 478 \\
\hline Lycopene & 446 & 472 & 503 \\
\hline$\alpha-$ carotene & 423 & 444 & 473 \\
\hline$\beta-$ carotene & 450 & 476 \\
\hline
\end{tabular}

\subsection{Research Methodology}

\subsubsection{Chemicals and instruments:}

Lutein, dionized water, ethyl alcohol (USP grade), 1-pentanol 99\%, hydrochloric acid, and triethylamine (TEA). Hexane, dichloromethane, methanol, acetronitrile, all HPLC grade. High pressure liquid chromatography (HPLC) system equipment used for carotenoid analysis: Waters 2690 couple to a photodiode array detector (PDA) Waters 996. High pressure liquid chromatography (HPLC) system used for standard purification: LDC/Milton Roy CM 4000 multiple solvent delivery system, Thermo Finnigan Spectra System UV 1000 detector. Ultraviolet-visible (UV-vis) spectrophotometer Cary 100 Bio.

\subsubsection{Subjects:}

Study I: 32 subjects (15 males and 17 females) were recruited from the FIU community for the study. Subjects were randomly assigned in one of the three groups: Group P1 was given placebo pills, and consisted of 10 subjects (4 males and 6 females). Group L1 was supplemented with pills containing lutein ( $20 \mathrm{mg} /$ day), and consisted of 11 subjects (5 males and 6 females). 
Group L2 was supplemented with diacetate lutein pills (20 mg/day), and consisted of 11 subjects (6 males and 5 females). Blood samples were collected before the supplementation and every 6 weeks after supplementation for 24 weeks in total. Macular pigment optical density was measured by heterochromatic flicker photometry before supplementation and every 6 weeks after supplementation for each subject.

Study II: 29 subjects (22 males and 7 females) were recruited from the FIU community, as well for this study. Subjects were randomly assigned in one of the three groups: Group P2 was given placebo pills, and consisted of 9 subjects (6 males and 3 females). Group L3 was supplemented with pills containing lutein diester $(9 \mathrm{mg} /$ day), and consisted of 10 subjects (8 males and 2 females). Group L4 was supplemented with pills containing lutein diester (9 $\mathrm{mg}$ /day), and additional, low level nutritional components, including lactoferrin, cassis extract, eyebright extract, royal jelly, coenzyme Q10. Study II consisted of 10 subjects (8 males and 2 females). Blood samples were collected before the supplementation and every 6 weeks after supplementation for 24 weeks in total. Macular pigment optical density was measure by heterochromatic flicker photometry before supplementation and every 6 weeks after supplementation for each subject.

\subsubsection{Internal standard preparation:}

Monopentyl lutein ether (MPL) was used as an internal standard for this study. In a flask, we dissolved $5 \mathrm{mg}$ of lutein in $20 \mathrm{~mL}$ of 1-pentanol, and $1 \% \mathrm{v} / \mathrm{v}$ of hydrochloric acid was added. The reaction was placed for 3 hours in the dark and under stirring. Then, the solution was extracted three times with a solution of dichloromethane and deionized water (1:1). The product was concentrated and redissolved with ethanol to be purified by reversed phase HPLC. The concentration was determined by UV-visible spectrophotometry. 


\subsubsection{Serum extraction:}

Blood samples were collected from the subjects and the serum was separated by centrifugation. The serum samples were collected in $2 \mathrm{~mL}$ polypropylene vials, labeled, and stored at $-80^{\circ} \mathrm{C}$ in the freezer. The carotenoids were extracted according to previous published technique [53]. The sample was thawed at room temperature and vortexed. An aliquot of $200 \mu \mathrm{L}$ of serum was placed in a glass tube, $35 \mu \mathrm{L}$ of MPL and $2 \mathrm{~mL}$ of a mixture of ethanol/dionized water (1:1) was added to the tube. The tube was vortexed for 30 seconds and carotenoids were extracted three times with $2 \mathrm{~mL}$ of hexane. For every extraction the tube was vortexed and centrifuged for 5 minutes. The top layer of the separation was removed every time and collected in a $2 \mathrm{~mL}$ vial. The aliquot obtained was dried under nitrogen gas flow. The sample was redissolved in $50 \mu \mathrm{L}$ of ethanol and transfered to a vial for HPLC injection.

\subsubsection{Carotenoid analysis:}

The samples were analyzed by reversed phase HPLC, using a Phenomenex ODS ultracarb $3 \mu \mathrm{m}$ column (20) of $250 \times 4.60 \mathrm{~mm}$. The mobile phase was a mixture of acetonitrile/methanol/TEA ( $85 \%: 15 \%: 0.1 \% \mathrm{v} / \mathrm{v})$. The flow rate was $1 \mathrm{~mL} /$ minute and the sample was run at $30^{\circ} \mathrm{C}$ for 70 minutes. The results were obtained from the chromatogram and peaks were labeled consistent with their retention time.

\subsubsection{MPOD measurements $[59,60]$ :}

The heterochromatic flicker photometry (HFP) is the method used to determine the MPOD. It can determine the spectral sensitivity of a human eye. In heterochromatic flicker photometry, lights of different spectral composition are alternated, and the subject has to adjust their relative radiances until the sensation of flicker is minimized or abolished. The two spectral mixtures are then defined as of equal luminance for that observer. To define a spectral sensitivity 
function, different monochromatic lights (460 and $540 \mathrm{~nm}$, in this case) are alternated with some standard. The flicker frequency is also an important parameter for the response to be reliable. The response defines the human photopic luminosity function that represents the measure of the average human visual sensitivity. The function is measure in two visual fields: small $\left(2^{\circ}\right)$ and large $\left(10^{\circ}\right)$. When it is measured using the small visual field it has an effect on the macular pigment but is quite insignificant in the large field. Therefore the difference of both functions gives a measure of the MPOD.

The subject's eye was covered with an eye patch and the subject was allowed to fix the focus of the stimulus with a hand-controlled button. Looking at the center of the stimulus the subject adjusts the setting until a flicker null is perceived and the wedge setting is recorded. This method is repeated five times in each: the small and large visual field. The MPOD of each subject were measured before and six weeks each time after supplementation for a total of 24 weeks period.

\subsection{Results:}

\subsubsection{Characterization of the standard}

The UV-Visible spectrum shown below corresponds to the standard prepared.

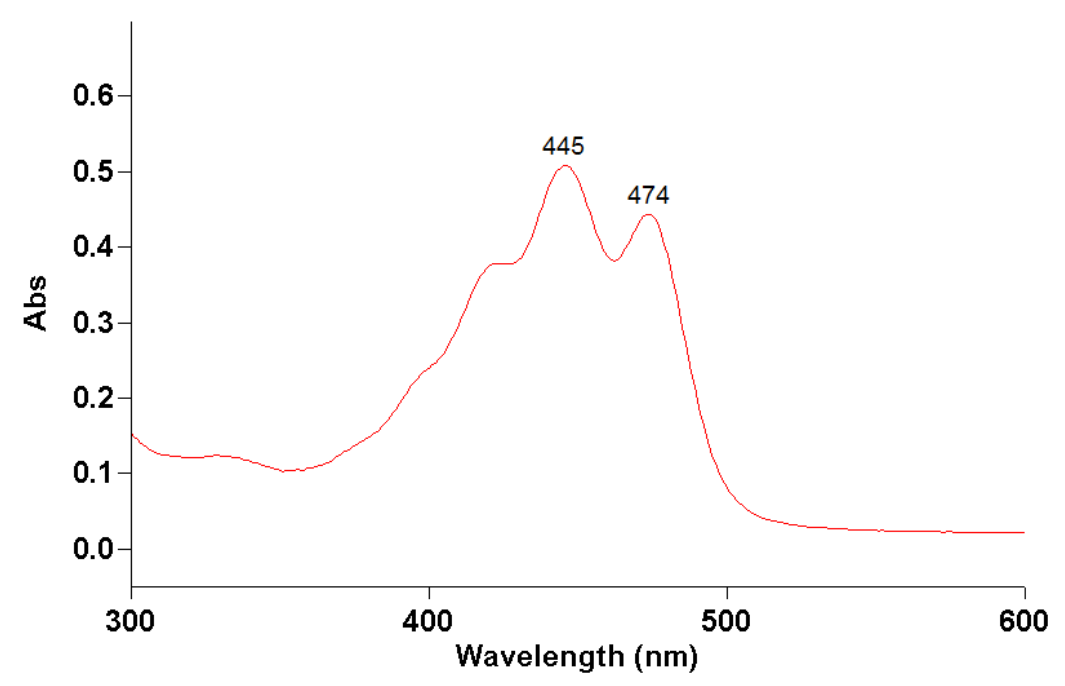

Figure 38: Monopentyl lutein UV-visible spectrum. 
The spectrum is the same as that of lutein. The concentration of the standard was determinate using the Beer-Lambert law with the absorbance response at 450nm.

\subsubsection{Baseline}

The chromatogram below is a characteristic serum response of one of the subjects in the studies. The peaks were assigned according to their UV-visible spectra.

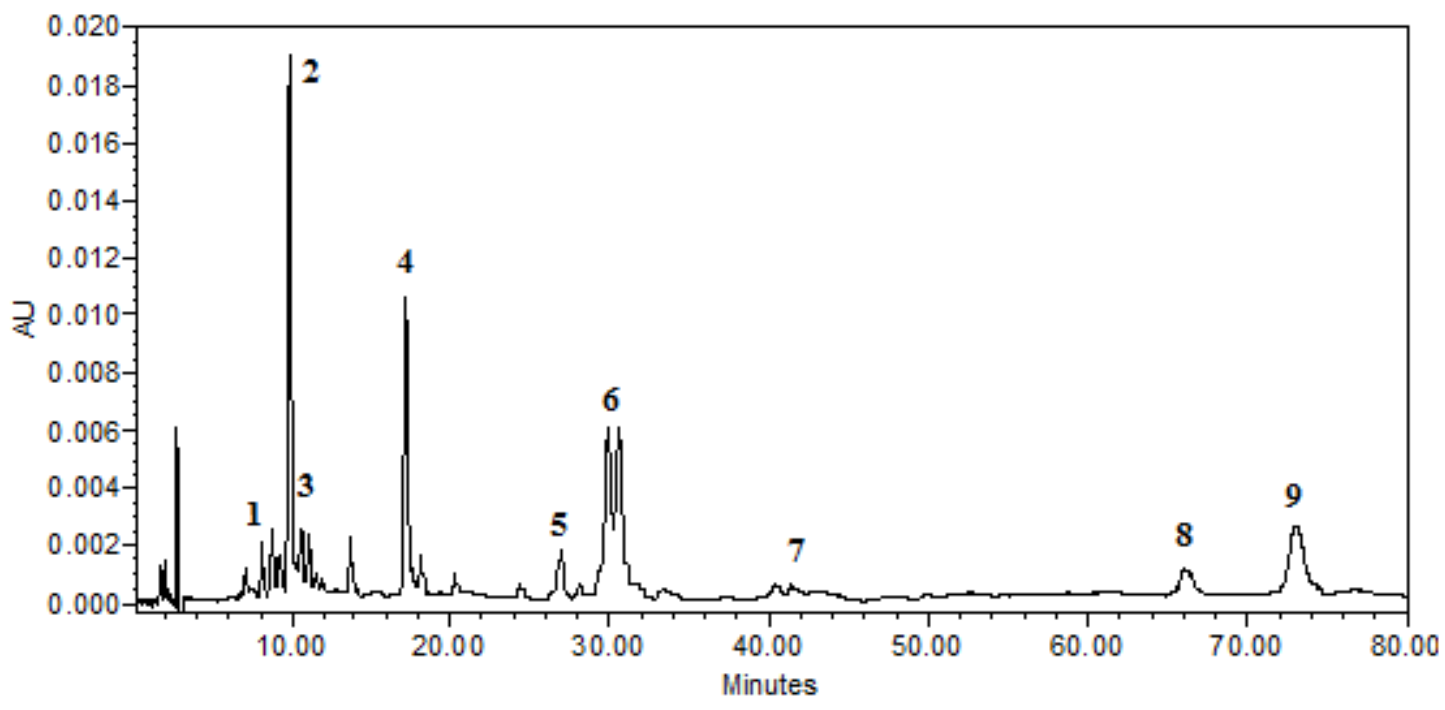

Figure 39: HPLC chromatogram of serum from subject before supplementation.

1) lutein metabolites. 2) lutein. 3) zeaxanthin. 4) $\alpha$-cryptoxanthin. 5) $\beta$-cryptoxanthin. 6) MPL. 7)lycopene. 8) $\alpha$-carotene. 9) $\beta$-carotene.

The picture below shows the expanded chromatogram from $0-12$ minutes. The macula pigment carotenoids peaks are shown. 


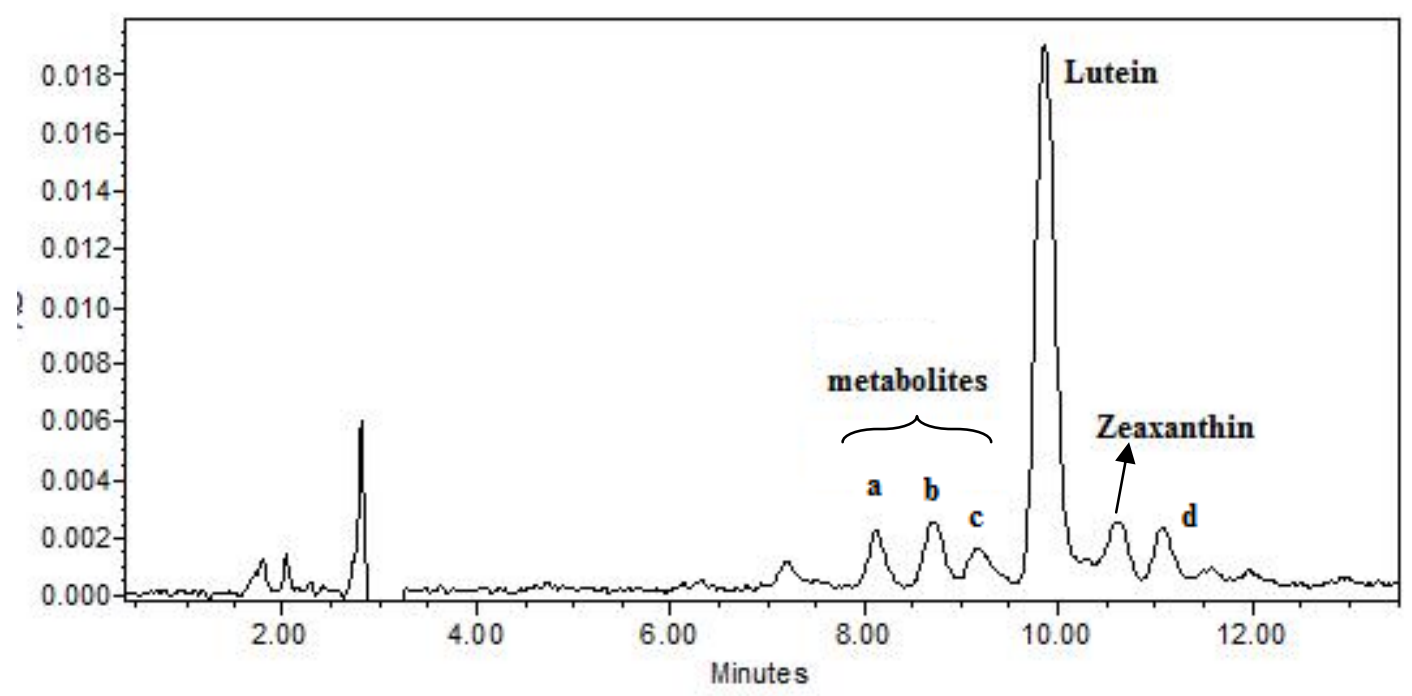

Figure 40: Expanded HPLC chromatogram of serum from subject before supplementation a) unknown. b) oxolutein. c) unknown d) $9 \mathrm{Z}$ lutein.

Table 32: Retention time of carotenoids in human serum.

\begin{tabular}{|c|c|}
\hline Carotenoid & Retention time (min) \\
\hline Lutein metabolites & $8.00-9.00$ \\
\hline Lutein & 9.85 \\
\hline Zeaxanthin & 10.61 \\
\hline$\alpha-$ cryptoxanthin & 17.13 \\
\hline$\beta-$ cryptoxanthin & 26.88 \\
\hline Monopentyl lutein ether (MPL) & 30.61 \\
\hline Lycopene & 40.35 \\
\hline$\alpha-$ carotene & 66.21 \\
\hline$\beta-$ carotene & 73.08 \\
\hline
\end{tabular}

The distribution of the carotenoids in serum at baseline for all the groups that participate in both studies is shown below (for numeric values, refer to table 34). 


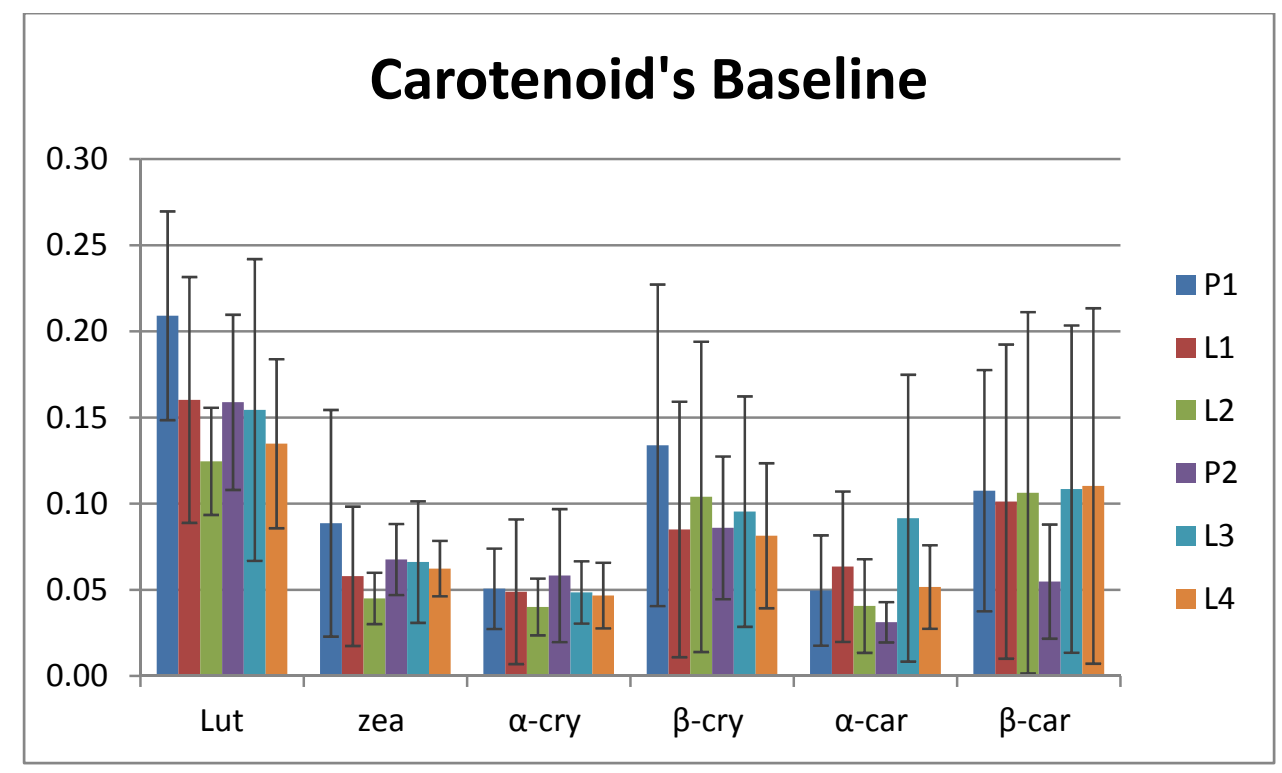

Figure 41: Distribution of the carotenoids at baseline according to the group. P1,placebo study 1. P2, placebo study II. L1 and L2, correspond to study I.

L3 and L4, correspond to study II.

The ratios for lutein/zeaxanthin at baseline for all the studied groups vary between 2.2 to 2.8 .

Table 33: Lutein/ zeaxanthin ratios for each study group.

\begin{tabular}{|l|r|r|r|r|r|r|}
\hline & \multicolumn{1}{|c|}{ P1 } & \multicolumn{1}{c|}{ L1 } & \multicolumn{1}{c|}{ L2 } & \multicolumn{1}{c|}{ P2 } & \multicolumn{1}{c|}{ L3 } & \multicolumn{1}{c|}{ L4 } \\
\hline $\mathbf{L} / \mathbf{Z}$ & 2.36 & 2.77 & 2.77 & 2.35 & 2.33 & 2.16 \\
\hline
\end{tabular}

Table 34: Characteristic serum concentrations of carotenoids at baseline.

\begin{tabular}{|l|c|c|c|c|c|c|}
\cline { 2 - 7 } \multicolumn{1}{c|}{} & \multicolumn{3}{c|}{ Study I } & \multicolumn{3}{c|}{ Study II } \\
\hline $\begin{array}{l}\text { Carotenoid } \\
\text { concentration/ } \\
\text { study group }\end{array}$ & $\mathrm{P} 1$ & $\mathrm{~L} 1$ & $\mathrm{~L} 2$ & $\mathrm{P} 2$ & $\mathrm{~L} 3$ & $\mathrm{~L} 4$ \\
\hline $\begin{array}{l}\text { Lutein } \\
(\mathrm{ng} / \mu \mathrm{L})\end{array}$ & $0.21 \pm 0.06$ & $0.16 \pm 0.07$ & $0.12 \pm 0.03$ & $0.16 \pm 0.05$ & $0.15 \pm 0.09$ & $0.13 \pm 0.05$ \\
\hline $\begin{array}{l}\text { Zeaxanthin } \\
(\mathrm{ng} / \mu \mathrm{L})\end{array}$ & $0.09 \pm 0.07$ & $0.06 \pm 0.04$ & $0.05 \pm 0.01$ & $0.07 \pm 0.02$ & $0.07 \pm 0.04$ & $0.06 \pm 0.02$ \\
\hline $\begin{array}{l}\alpha-\text {-crytoxanthin } \\
(\mathrm{ng} / \mu \mathrm{L})\end{array}$ & $0.05 \pm 0.02$ & $0.05 \pm 0.04$ & $0.04 \pm 0.02$ & $0.06 \pm 0.04$ & $0.05 \pm 0.02$ & $0.05 \pm 0.02$ \\
\hline $\begin{array}{l}\beta \text {-cryptoxanthin } \\
(\mathrm{ng} / \mu \mathrm{L})\end{array}$ & $0.13 \pm 0.09$ & $0.09 \pm 0.07$ & $0.10 \pm 0.09$ & $0.09 \pm 0.04$ & $0.10 \pm 0.07$ & $0.08 \pm 0.04$ \\
\hline $\begin{array}{l}\alpha-\text {-carotene } \\
(\mathrm{ng} / \mu \mathrm{L})\end{array}$ & $0.05 \pm 0.03$ & $0.06 \pm 0.05$ & $0.04 \pm 0.03$ & $0.03 \pm 0.01$ & $0.09 \pm 0.08$ & $0.05 \pm 0.02$ \\
\hline $\begin{array}{l}\beta-\text {-carotene } \\
(\mathrm{ng} / \mu \mathrm{L})\end{array}$ & $0.11 \pm 0.07$ & $0.10 \pm 0.10$ & $0.11 \pm 0.10$ & $0.05 \pm 0.03$ & $0.11 \pm 0.09$ & $0.11 \pm 0.10$ \\
\hline
\end{tabular}


No statistical difference was found between any of the groups at the baseline level when analyzed the data by one-way ANOVA $(\mathrm{p}>0.60)$.

\subsubsection{Supplementation Response}

The supplementation responses were recorded every 6 weeks for a period of 24 weeks.

For a better understanding the data is presenting by groups.

\section{Study I:}

\section{P1 group (placebo)}

Table 35: Carotenoid concentrations in $\mathrm{P} 1$ throughout the study period (mean \pm standard deviation).

\begin{tabular}{|c|c|c|c|c|c|c|}
\hline Week & $\begin{array}{c}\text { Lut } \\
(\mathrm{ng} / \mu \mathrm{L})\end{array}$ & $\begin{array}{c}\text { zea } \\
(\mathrm{ng} / \mu \mathrm{L})\end{array}$ & $\begin{array}{c}\alpha \text {-cry } \\
(\mathrm{ng} / \mu \mathrm{L})\end{array}$ & $\begin{array}{c}\beta \text {-cry } \\
(\mathrm{ng} / \mu \mathrm{L})\end{array}$ & $\begin{array}{c}\alpha \text {-car } \\
(\mathrm{ng} / \mu \mathrm{L})\end{array}$ & $\begin{array}{c}\beta \text {-car } \\
(\mathrm{ng} / \mu \mathrm{L})\end{array}$ \\
\hline $\mathbf{6}$ & $0.19 \pm 0.06$ & $0.07 \pm 0.03$ & $0.05 \pm 0.03$ & $0.15 \pm 0.11$ & $0.03 \pm 0.02$ & $0.09 \pm 0.08$ \\
$\mathbf{1 2}$ & $0.23 \pm 0.11$ & $0.07 \pm 0.04$ & $0.07 \pm 0.05$ & $0.13 \pm 0.09$ & $0.04 \pm 0.02$ & $0.05 \pm 0.06$ \\
$\mathbf{1 8}$ & $0.20 \pm 0.11$ & $0.06 \pm 0.03$ & $0.08 \pm 0.06$ & $0.09 \pm 0.07$ & $0.03 \pm 0.02$ & $0.04 \pm 0.04$ \\
$\mathbf{2 4}$ & $0.15 \pm 0.06$ & $0.06 \pm 0.02$ & $0.06 \pm 0.04$ & $0.09 \pm 0.09$ & $0.06 \pm 0.07$ & $0.06 \pm 0.06$ \\
\hline
\end{tabular}

No statistical difference $(\mathrm{p}>0.09)$ was found between the baseline and the response after supplementation for all the carotenoids in this group. 


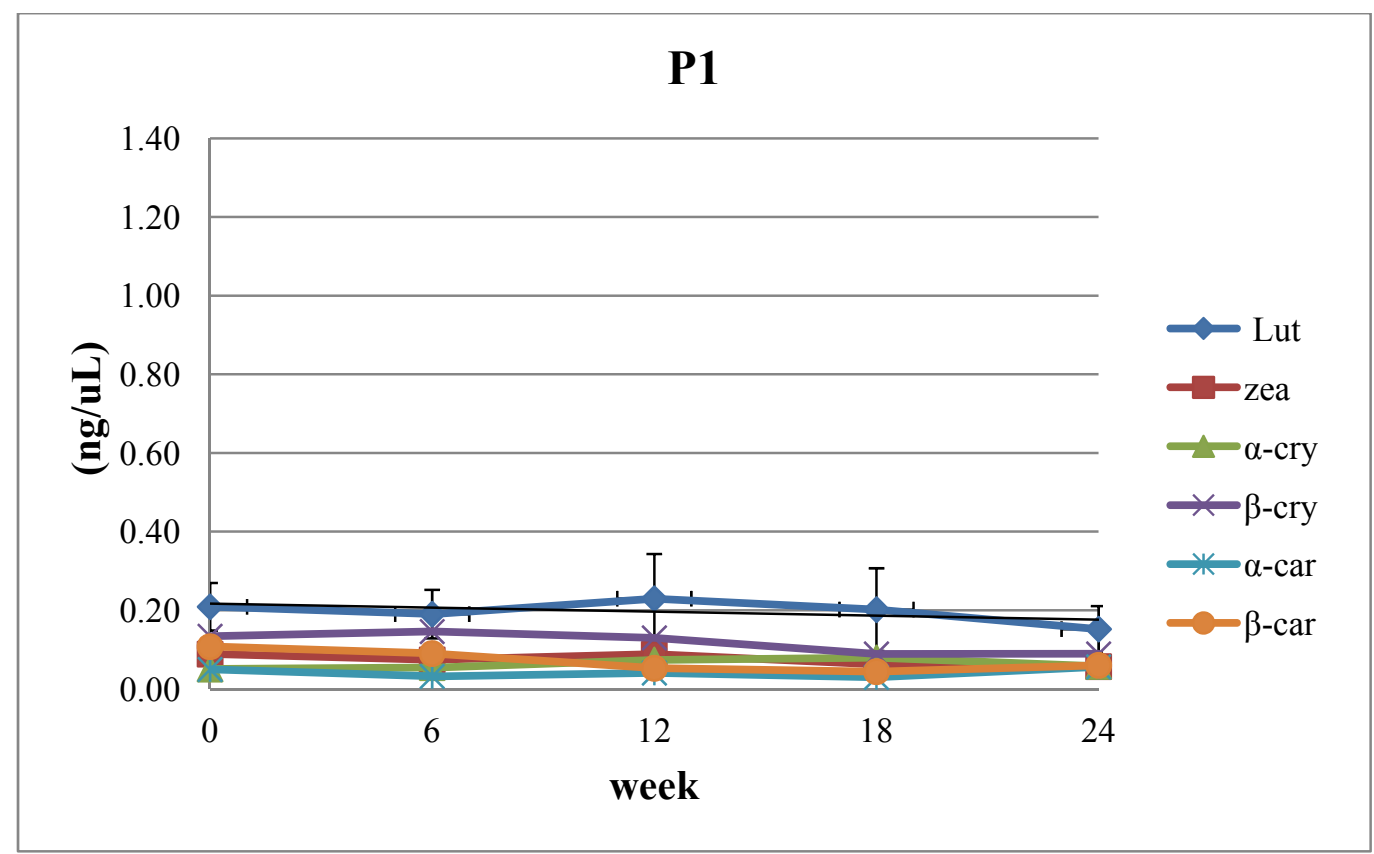

Figure 42: Comparison of carotenoids concentration in placebo group P1.

Numeric values are shown in table 4.

\section{L1 group}

Table 36: Carotenoid concentrations after supplementation with $\mathrm{L} 1$ (mean \pm standard deviation).

\begin{tabular}{|c|c|c|c|c|c|c|}
\hline Week & $\begin{array}{c}\text { Lut } \\
(\mathrm{ng} / \mu \mathrm{L})\end{array}$ & $\begin{array}{c}\text { zea } \\
(\mathrm{ng} / \mu \mathrm{L})\end{array}$ & $\begin{array}{c}\alpha \text {-cry } \\
(\mathrm{ng} / \mu \mathrm{L})\end{array}$ & $\begin{array}{c}\beta \text {-cry } \\
(\mathrm{ng} / \mu \mathrm{L})\end{array}$ & $\begin{array}{c}\alpha \text {-car } \\
(\mathrm{ng} / \mu \mathrm{L})\end{array}$ & $\begin{array}{c}\beta \text {-car } \\
(\mathrm{ng} / \mu \mathrm{L})\end{array}$ \\
\hline $\mathbf{6}$ & $0.67 \pm 0.35$ & $0.09 \pm 0.05$ & $0.05 \pm 0.02$ & $0.09 \pm 0.09$ & $0.03 \pm 0.01$ & $0.08 \pm 0.04$ \\
$\mathbf{1 2}$ & $0.72 \pm 0.37$ & $0.14 \pm 0.06$ & $0.06 \pm 0.03$ & $0.09 \pm 0.07$ & $0.08 \pm 0.08$ & $0.03 \pm 0.01$ \\
$\mathbf{1 8}$ & $0.68 \pm 0.46$ & $0.14 \pm 0.11$ & $0.09 \pm 0.05$ & $0.08 \pm 0.07$ & $0.06 \pm 0.04$ & $0.06 \pm 0.04$ \\
$\mathbf{2 4}$ & $0.57 \pm 0.28$ & $0.13 \pm 0.07$ & $0.06 \pm 0.03$ & $0.06 \pm 0.05$ & $0.05 \pm 0.06$ & $0.04 \pm 0.02$ \\
\hline
\end{tabular}




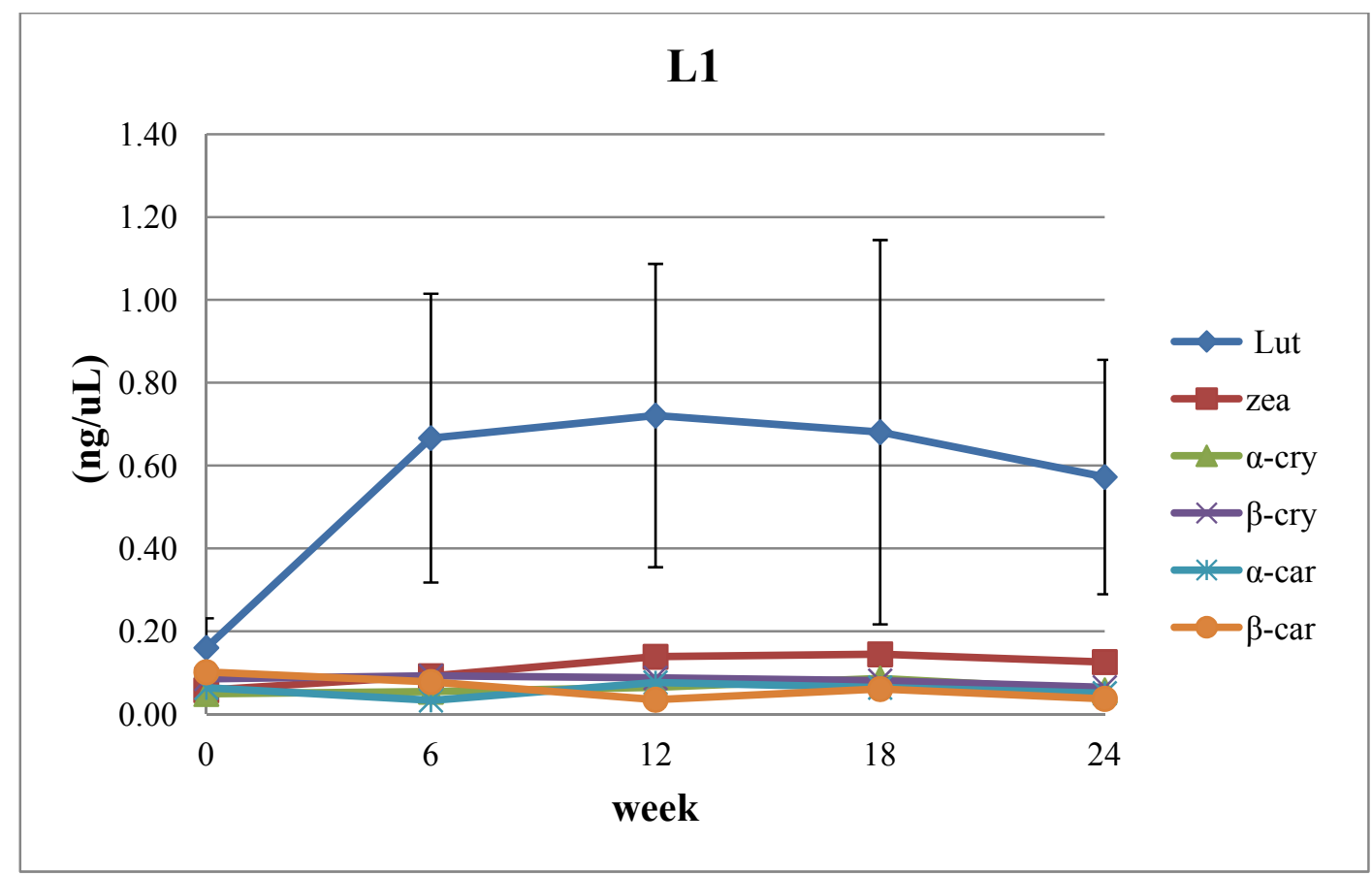

Figure 43: Comparison of carotenoids concentration in L1 group.

Numeric values are shown in table 5.

For lutein: $\mathrm{p}<0.001$, and for zeaxanthin: $\mathrm{p}<0.007$. There was a significant statistical difference when the subjects were compared to baseline. For the rest of the carotenoids: $p>0.20$, no statistical difference exists when compared to baseline.

\section{L2 group}

Table 37: Carotenoid concentrations after supplementation in L2 (mean \pm standard deviation).

\begin{tabular}{|c|c|c|c|c|c|c|}
\hline week & $\begin{array}{c}\text { Lut } \\
(\mathrm{ng} / \mu \mathrm{L})\end{array}$ & $\begin{array}{c}\text { zea } \\
(\mathrm{ng} / \mu \mathrm{L})\end{array}$ & $\begin{array}{c}\alpha \text {-cry } \\
(\mathrm{ng} / \mu \mathrm{L})\end{array}$ & $\begin{array}{c}\beta \text {-cry } \\
(\mathrm{ng} / \mu \mathrm{L})\end{array}$ & $\begin{array}{c}\alpha \text {-car } \\
(\mathrm{ng} / \mu \mathrm{L})\end{array}$ & $\begin{array}{c}\beta \text {-car } \\
(\mathrm{ng} / \mu \mathrm{L})\end{array}$ \\
\hline $\mathbf{6}$ & $0.65 \pm 0.37$ & $0.08 \pm 0.04$ & $0.05 \pm 0.02$ & $0.11 \pm 0.21$ & $0.03 \pm 0.02$ & $0.06 \pm 0.05$ \\
$\mathbf{1 2}$ & $0.76 \pm 0.58$ & $0.16 \pm 0.08$ & $0.07 \pm 0.02$ & $0.13 \pm 0.15$ & $0.01 \pm 0.01$ & $0.05 \pm 0.02$ \\
$\mathbf{1 8}$ & $0.69 \pm 0.60$ & $0.12 \pm 0.10$ & $0.07 \pm 0.04$ & $0.11 \pm 0.12$ & $0.03 \pm 0.01$ & $0.06 \pm 0.05$ \\
$\mathbf{2 4}$ & $0.60 \pm 0.40$ & $0.09 \pm 0.05$ & $0.06 \pm 0.03$ & $0.10 \pm 0.10$ & $0.04 \pm 0.02$ & $0.10 \pm 0.08$ \\
\hline
\end{tabular}




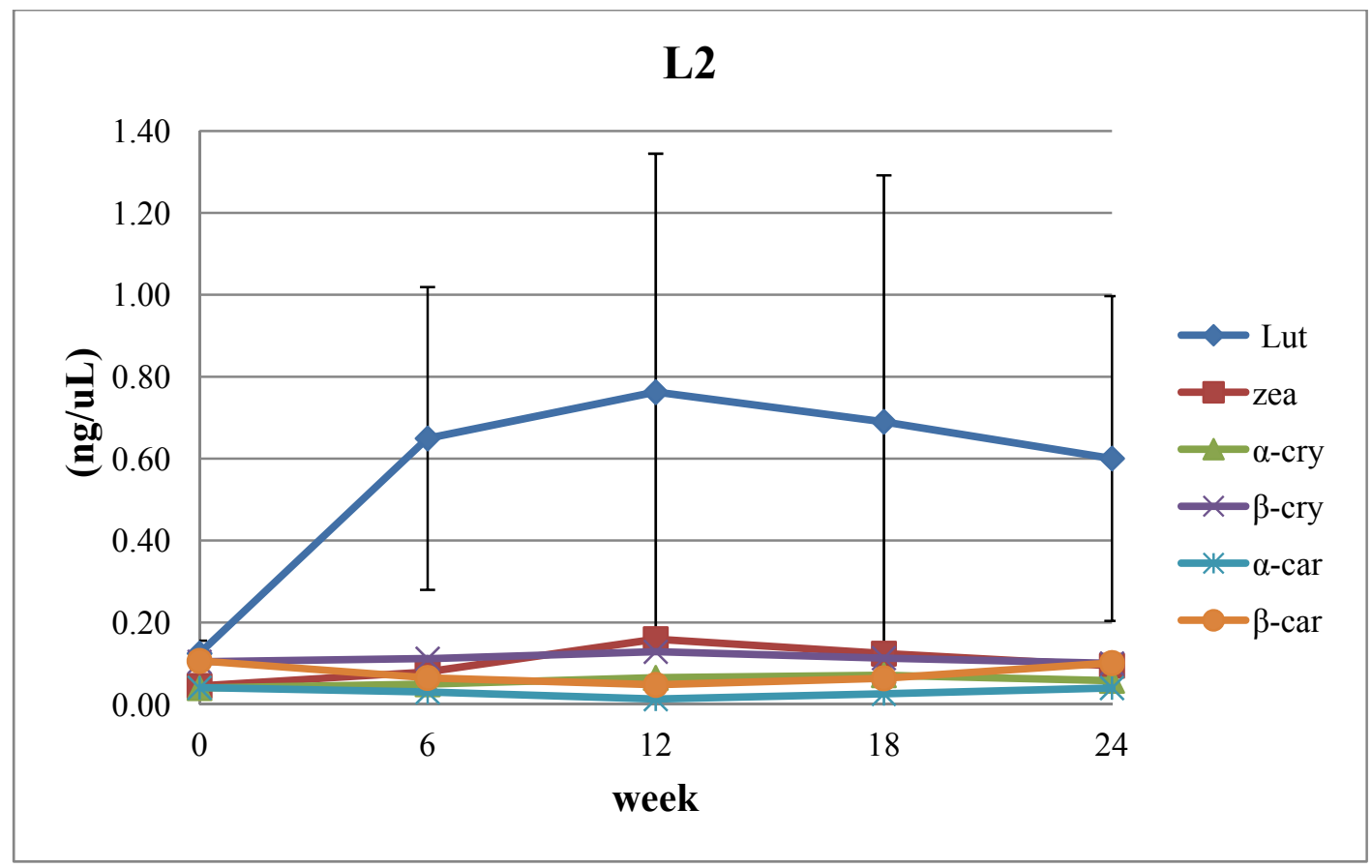

Figure 44: Comparison of carotenoids concentration in L2 group.

Numeric values are shown in table 6.

For lutein and for zeaxanthin: $\mathrm{p}<0.001$. There was a significant statistical difference when the subjects were compared to baseline. For the rest of the carotenoids (except for $\alpha$ cryptoxanthin) $: p>0.20$, no statistical difference exists when compared to baseline. For $\alpha-$ cryptoxanthin: $\mathrm{p}>0.04$.

\section{MPOD results:}

The MPOD rate for each study group is summarized in table 7:

Table 38: MPOD rates for each group (mean \pm standard deviation) in study I.

\begin{tabular}{|cccc|}
\hline & P1 & L1 & L2 \\
Rate of MPOD & $-0.0008 \pm 0.0014$ & $0.0017 \pm 0.0011$ & $0.0019 \pm 0.0005$ \\
increase \\
(AU/day)
\end{tabular}




\section{Study II:}

\section{P2 group (placebo)}

Table 39: Carotenoid concentrations after supplementation in $\mathrm{P} 2$ (mean \pm standard deviation).

\begin{tabular}{|c|c|c|c|c|c|c|}
\hline week & $\begin{array}{c}\text { Lut } \\
(\mathrm{ng} / \mu \mathrm{L})\end{array}$ & $\begin{array}{c}\text { zea } \\
(\mathrm{ng} / \mu \mathrm{L})\end{array}$ & $\begin{array}{c}\alpha \text {-cry } \\
(\mathrm{ng} / \mu \mathrm{L})\end{array}$ & $\begin{array}{c}\beta \text {-cry } \\
(\mathrm{ng} / \mu \mathrm{L})\end{array}$ & $\begin{array}{c}\alpha \text {-car } \\
(\mathrm{ng} / \mu \mathrm{L})\end{array}$ & $\begin{array}{c}\beta \text {-car } \\
(\mathrm{ng} / \mu \mathrm{L})\end{array}$ \\
\hline $\mathbf{6}$ & $0.16 \pm 0.08$ & $0.07 \pm 0.03$ & $0.05 \pm 0.04$ & $0.11 \pm 0.06$ & $0.04 \pm 0.04$ & $0.05 \pm 0.03$ \\
$\mathbf{1 2}$ & $0.18 \pm 0.05$ & $0.08 \pm 0.03$ & $0.06 \pm 0.04$ & $0.13 \pm 0.08$ & $0.05 \pm 0.01$ & $0.04 \pm 0.02$ \\
$\mathbf{1 8}$ & $0.19 \pm 0.10$ & $0.08 \pm 0.03$ & $0.07 \pm 0.06$ & $0.13 \pm 0.07$ & $0.04 \pm 0.00$ & $0.05 \pm 0.03$ \\
$\mathbf{2 4}$ & $0.17 \pm 0.07$ & $0.08 \pm 0.02$ & $0.07 \pm 0.07$ & $0.12 \pm 0.08$ & $0.03 \pm 0.01$ & $0.05 \pm 0.02$ \\
\hline
\end{tabular}

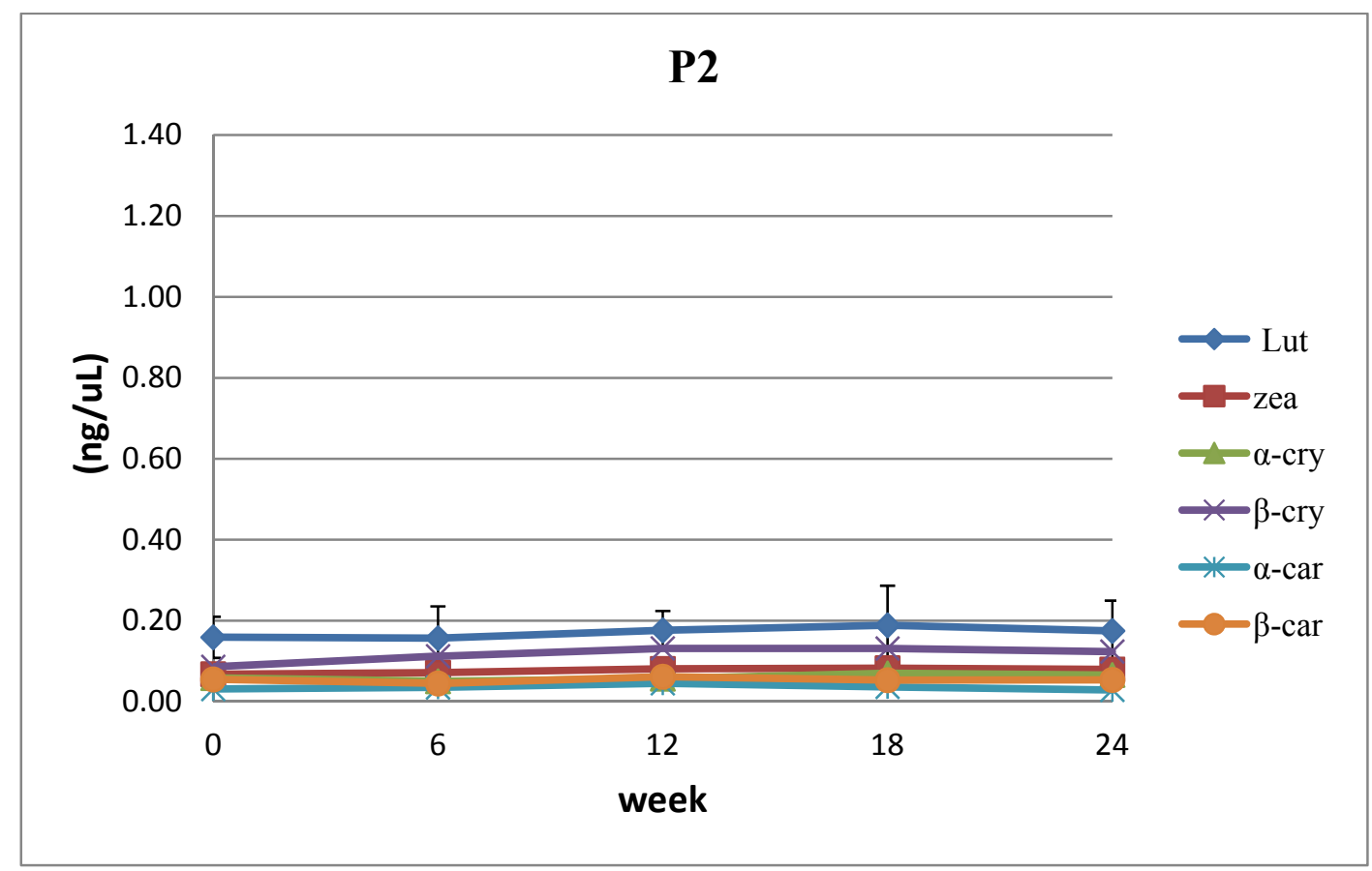

Figure 45: Comparison of carotenoids concentration in P2 group.

Numeric values are shown in table 7.

No statistical difference $(\mathrm{p}>0.10)$ was found between the baseline and the response after supplementation for all the carotenoids in this group. 


\section{L3 group}

Table 40: Carotenoid concentrations after supplementation in L3 (mean \pm standard deviation).

\begin{tabular}{|c|c|c|c|c|c|c|}
\hline week & $\begin{array}{c}\text { Lut } \\
(\mathrm{ng} / \mu \mathrm{L})\end{array}$ & $\begin{array}{c}\text { zea } \\
(\mathrm{ng} / \mu \mathrm{L})\end{array}$ & $\begin{array}{c}\alpha \text {-cry } \\
(\mathrm{ng} / \mu \mathrm{L})\end{array}$ & $\begin{array}{c}\beta \text {-cry } \\
(\mathrm{ng} / \mu \mathrm{L})\end{array}$ & $\begin{array}{c}\alpha \text {-car } \\
(\mathrm{ng} / \mu \mathrm{L})\end{array}$ & $\begin{array}{c}\beta \text {-car } \\
(\mathrm{ng} / \mu \mathrm{L})\end{array}$ \\
\hline $\mathbf{6}$ & $0.24 \pm 0.11$ & $0.07 \pm 0.03$ & $0.04 \pm 0.01$ & $0.12 \pm 0.07$ & $0.12 \pm 0.10$ & $0.09 \pm 0.08$ \\
$\mathbf{1 2}$ & $0.27 \pm 0.18$ & $0.08 \pm 0.03$ & $0.05 \pm 0.04$ & $0.10 \pm 0.05$ & $0.03 \pm 0.00$ & $0.05 \pm 0.02$ \\
$\mathbf{1 8}$ & $0.27 \pm 0.09$ & $0.10 \pm 0.03$ & $0.05 \pm 0.02$ & $0.11 \pm 0.05$ & $0.04 \pm 0.04$ & $0.07 \pm 0.06$ \\
$\mathbf{2 4}$ & $0.30 \pm 0.13$ & $0.10 \pm 0.04$ & $0.05 \pm 0.02$ & $0.12 \pm 0.05$ & $0.03 \pm 0.00$ & $0.05 \pm 0.03$ \\
\hline
\end{tabular}

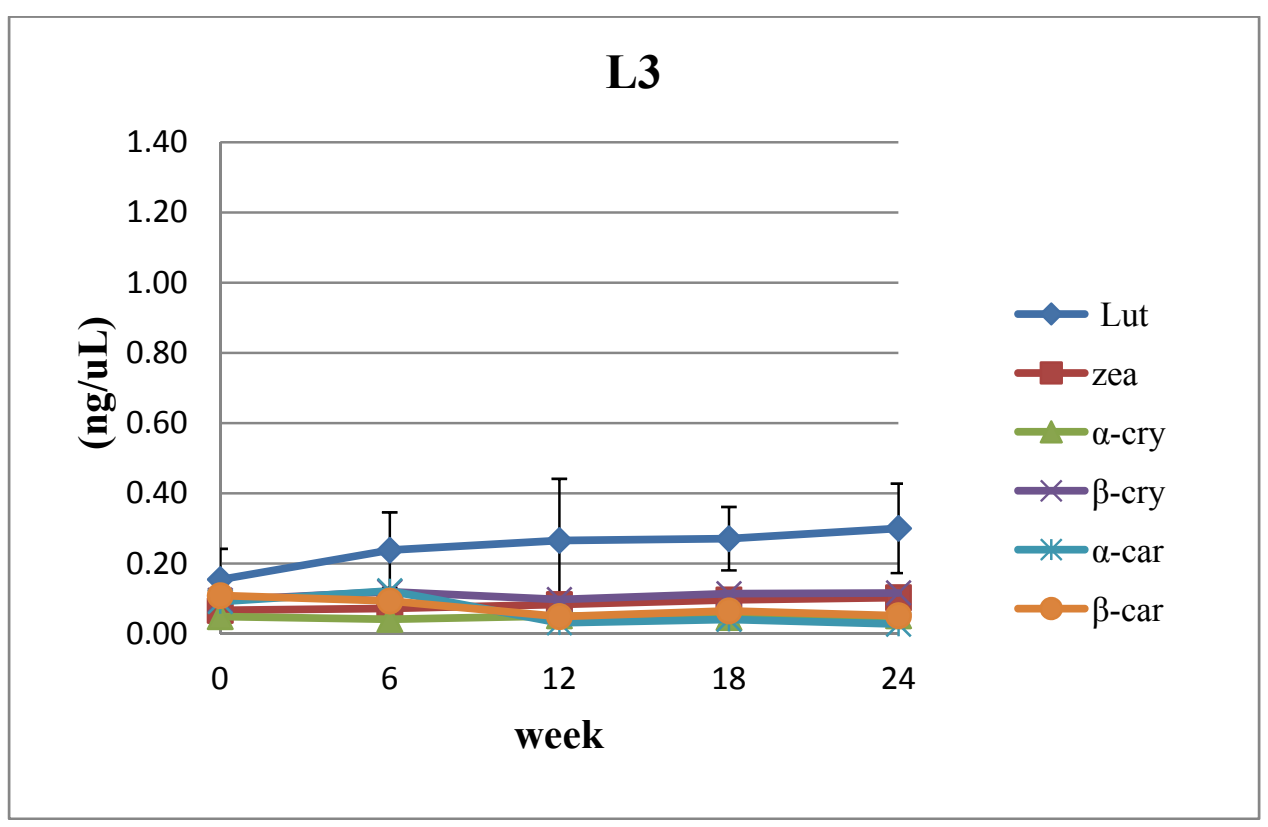

Figure 46: Comparison of carotenoids concentration in L3 group.

Numeric values are shown in table 8.

For lutein: $\mathrm{p}<0.004$, there was a significant statistical difference when the subjects were compared to baseline. For zeaxanthin: $p>0.08$, and for the rest of the carotenoids: $p>0.20$, no statistical difference exists when compared to baseline. 


\section{L4 group}

Table 41: Carotenoid concentrations after supplementation in L4 (mean \pm standard deviation).

\begin{tabular}{|c|c|c|c|c|c|c|}
\hline week & $\begin{array}{c}\text { Lut } \\
(\mathrm{ng} / \mu \mathrm{L})\end{array}$ & $\begin{array}{c}\text { zea } \\
(\mathrm{ng} / \mu \mathrm{L})\end{array}$ & $\begin{array}{c}\alpha \text {-cry } \\
(\mathrm{ng} / \mu \mathrm{L})\end{array}$ & $\begin{array}{c}\beta \text {-cry } \\
(\mathrm{ng} / \mu \mathrm{L})\end{array}$ & $\begin{array}{c}\alpha \text {-car } \\
(\mathrm{ng} / \mu \mathrm{L})\end{array}$ & $\begin{array}{c}\beta \text {-car } \\
(\mathrm{ng} / \mu \mathrm{L})\end{array}$ \\
\hline $\mathbf{6}$ & $0.61 \pm 0.22$ & $0.10 \pm 0.02$ & $0.06 \pm 0.03$ & $0.10 \pm 0.08$ & $0.07 \pm 0.02$ & $0.09 \pm 0.04$ \\
$\mathbf{1 2}$ & $0.59 \pm 0.39$ & $0.10 \pm 0.06$ & $0.06 \pm 0.03$ & $0.11 \pm 0.07$ & N.D. & $0.07 \pm 0.07$ \\
$\mathbf{1 8}$ & $0.61 \pm 0.23$ & $0.12 \pm 0.05$ & $0.06 \pm 0.03$ & $0.12 \pm 0.07$ & $0.03 \pm 0.00$ & $0.09 \pm 0.08$ \\
$\mathbf{2 4}$ & $0.62 \pm 0.40$ & $0.13 \pm 0.07$ & $0.06 \pm 0.02$ & $0.12 \pm 0.06$ & $0.04 \pm 0.01$ & $0.05 \pm 0.04$ \\
\hline
\end{tabular}

N.D. $=$ no data were recorded.

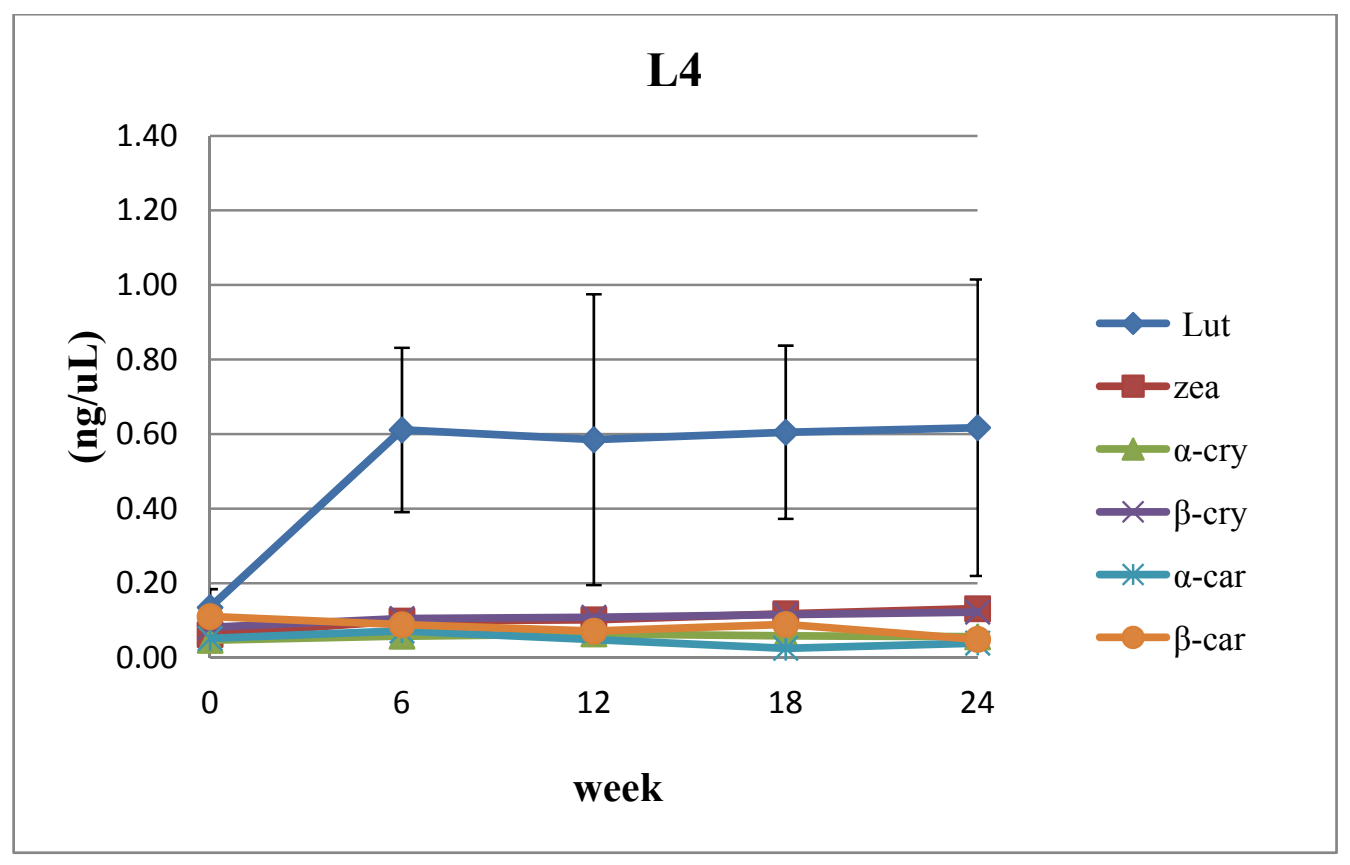

Figure 47: Comparison of carotenoids concentration in L4 group.

Numeric values are shown in table 9.

For lutein: $\mathrm{p}<0.001$, and for zeaxanthin: $\mathrm{p}<0.004$. There was a significant statistical difference when the subjects were compared to baseline. For the rest of the carotenoids: $p>0.10$, no statistical difference exists when compared to baseline. 


\section{MPOD results:}

The MPOD increase for each study group is summarized in table 11:

Table 42: MPOD rates for each group (mean \pm standard deviation) in study II.

\begin{tabular}{|cccc|}
\hline & P2 & L3 & L4 \\
$\begin{array}{c}\text { Rate of MPOD } \\
\text { increase } \\
\text { (AU/day) }\end{array}$ & $0.0011 \pm 0.0008$ & $0.0011 \pm 0.0012$ & $0.0026 \pm 0.0006$ \\
\hline
\end{tabular}

There is no difference between the placebo group and the L3 group.

\subsection{Discussion:}

\subsubsection{Study I:}

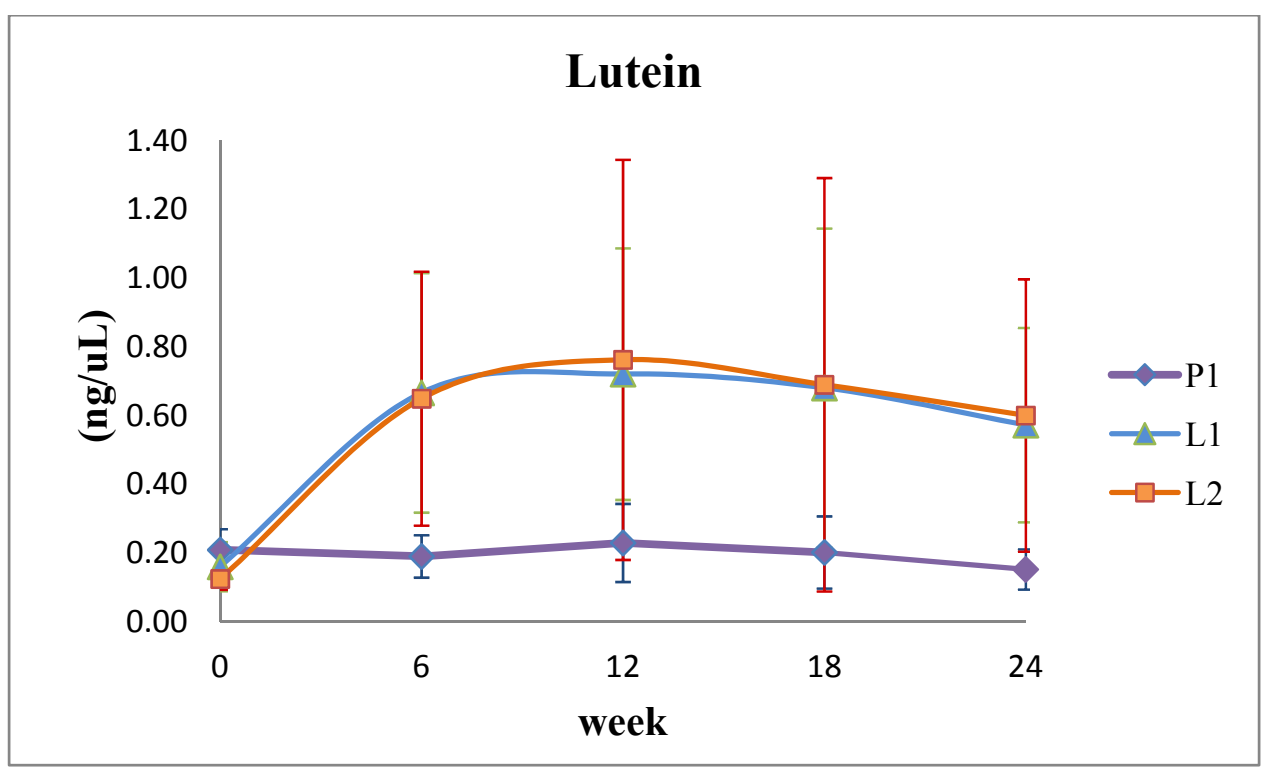

Figure 48: Comparison of lutein concentration in serum for study I.

Figure 48 shows a comparison for the lutein concentration in serum between the three groups. According to the values during the supplementation period, the concentration of lutein in 
the case of the supplemented groups increases within the first 6 weeks and remains almost constant for the rest of the study (until week $24^{\text {th }}$ is completed). It reached its maximum value during the $12^{\text {th }}$ week of supplementation for both L1 and L2. The placebo group remains constant as expected. The error bars in both cases (L1 y L2) are more than $50 \%$ of the average of the concentration value in each period of time. This variability is caused to the differences of the concentration of lutein in serum for each subject. Lutein concentrations vary from one subject to another as a consequence of their diet. The ANOVA run for the supplemented groups for the average concentration of lutein in serum during supplementation demonstrates that the results for L1 and L2 are highly significant different $(\mathrm{p}<0.0002)$ from baseline values.

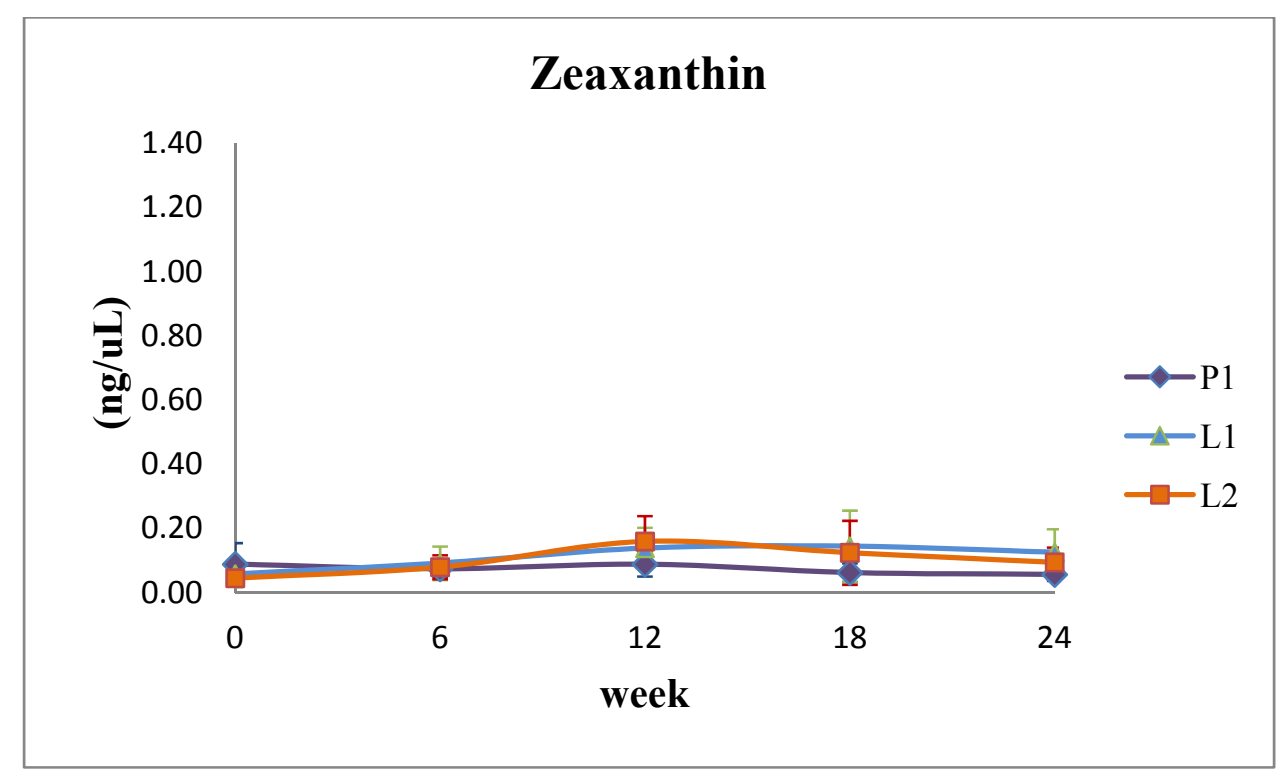

Figure 49: Comparison of zeaxanthin concentration in serum for study I.

Figure 49 shows the comparison for zeaxanthin in the study. The concentrations are smaller since the ratio $\mathrm{L} / \mathrm{Z}$ in human serum is $2-3$. For the ANOVA run like in the case of lutein the results were: $\mathrm{L} 1 \mathrm{p}<0.006$, and for $\mathrm{L} 2 \mathrm{p}<0.0005$, as similar to the lutein case shown above the 
error is around $50 \%$ or more. Again, is because of the variability of the subjects within the groups.

Table 43 summarizes the average concentration for each group after supplementation (weeks 6, 12, 18 and 24) in comparison with the baseline concentrations for lutein and zeaxanthin.

Table 43: Concentrations for lutein and zeaxanthin in each group before and after supplementation in study I.

\begin{tabular}{|cccc|ccc|}
\hline \multicolumn{3}{|c|}{ Lutein $(\mathrm{ng} / \mu \mathrm{L})$} & \multicolumn{3}{c|}{ Zeaxanthin $(\mathrm{ng} / \mu \mathrm{L})$} \\
& $\mathrm{P} 1$ & $\mathrm{~L} 1$ & $\mathrm{~L} 2$ & $\mathrm{P} 1$ & $\mathrm{~L} 1$ & $\mathrm{~L} 2$ \\
Baseline & $0.21 \pm 0.06$ & $0.16 \pm 0.07$ & $0.12 \pm 0.03$ & $0.09 \pm 0.07$ & $0.06 \pm 0.04$ & $0.05 \pm 0.01$ \\
Average & $0.19 \pm 0.02$ & $0.66 \pm 0.05$ & $0.68 \pm 0.10$ & $0.07 \pm 0.01$ & $0.13 \pm 0.02$ & $0.11 \pm 0.02$ \\
\hline
\end{tabular}

In Figure 50 we can see a comparison of the average concentrations for lutein and zeaxanthin after supplementation by groups. The increase of lutein for group L1 is around four times more, and for group L2 is more than five times of the corresponding to the baseline level. This high increase can be attributed to the fact that the supplementation given was of $20 \mathrm{mg}$ of free lutein in each case (L1 and L2). The only difference between them is the formula used for each pill. For zeaxanthin the amounts double the amount of the baseline. 


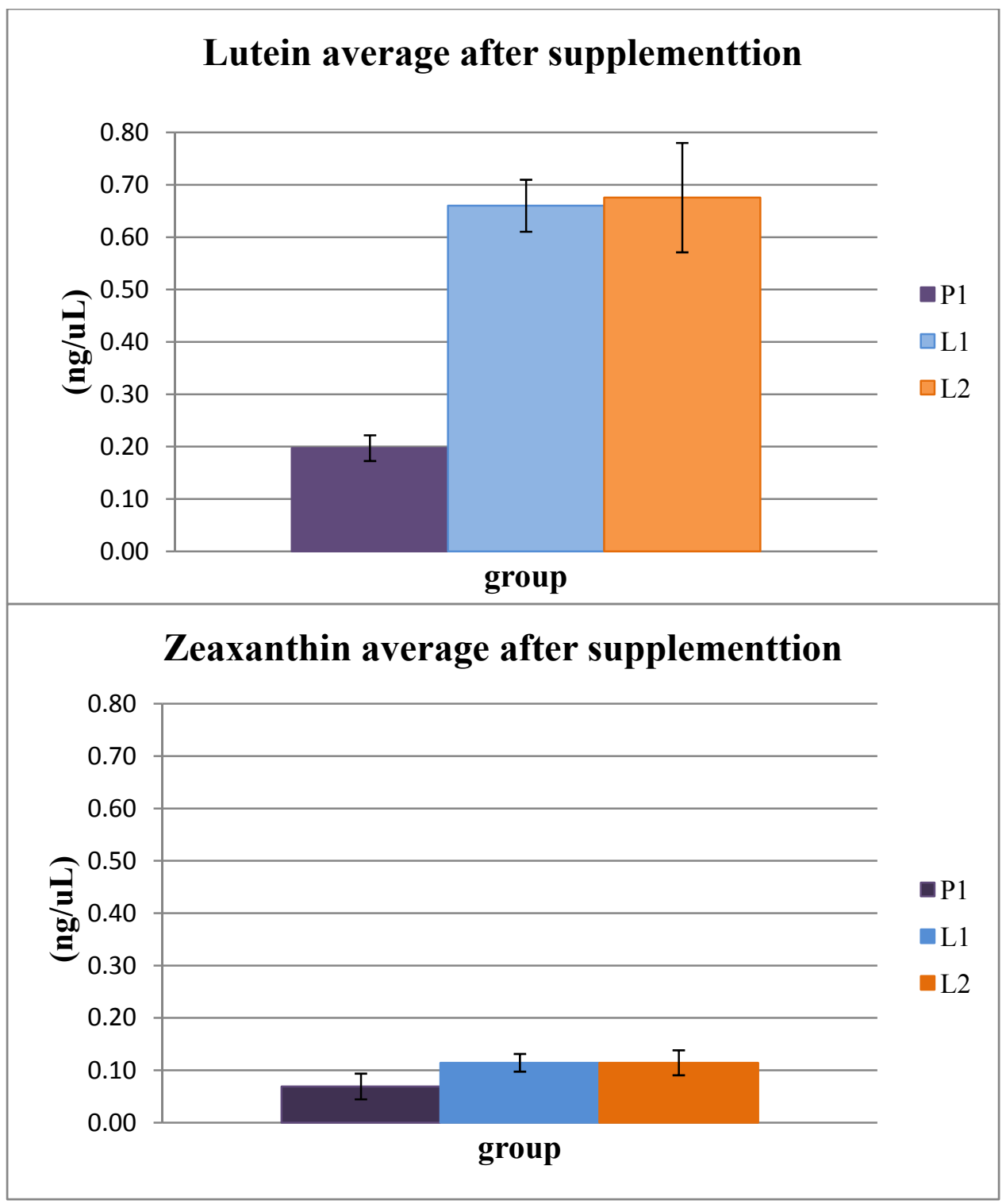

Figure 50: Average concentrations for lutein and zeaxanthin after supplementation in serum for study I.

The rate of change of MPOD was determined as the slope of a linear fit to the MPOD measures during the 24 weeks of supplementation (see table 38) and fixed to baseline of each group.. The standards deviation of the MPOD mean rate for groups L1 and P1 are larger (more than 60\%) than for group L2 (approximately 25\%). This numbers evidence the variability 
between subjects in the groups. The levels of MPOD are smaller in comparison with the serum response. The correlation between the average serum levels after supplementation and MPOD rates is almost perfectly linear $\left(\mathrm{R}^{2}=0.9997\right)$ for this study, but no significant statistical difference between the groups was found.

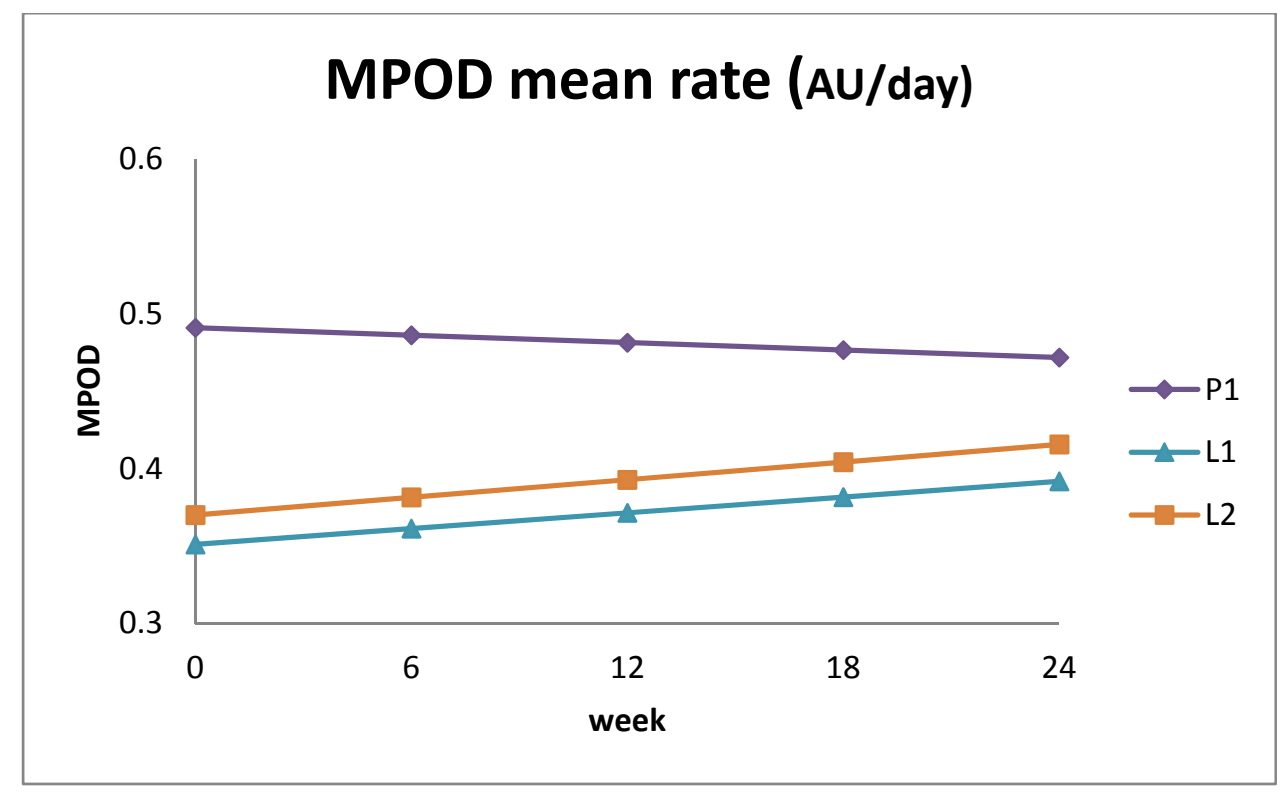

Figure 51: Comparison of mean rate of MPOD for study I.

\subsubsection{Study II:}

Figure 52 illustrate the comparison of lutein concentration in serum between the study groups. The placebo group remains fairly constant along the study period. As seen in the first study, the concentration of lutein in serum for the supplemented groups increases during the first 6 weeks and remains slightly constant for the next 18 weeks. For the L4 group the maximum value is reached at week sixth and the value remains constant after. For the L3 group the concentration continued increasing approximately $12 \%$ every six weeks after the sixth week. 
Once again, the error bars appear to be around $50 \%$ for the supplemented groups because of the variability within the subjects. The ANOVA run for group L3 $(p<0.004)$ and for L4 $(p<0.0002)$ evidence that the results for the lutein concentrations are statistically different form the baseline values.

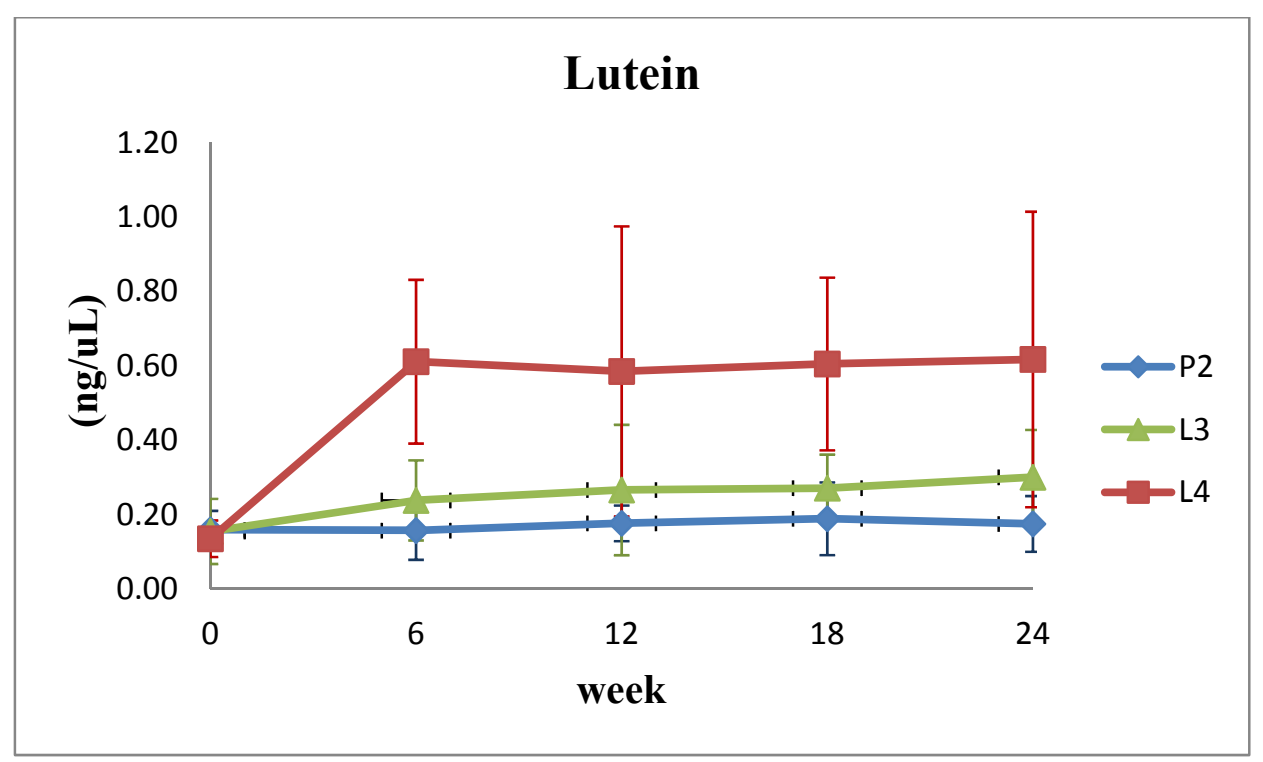

Figure 52: Comparison of lutein concentration in serum for study II.

Figure 53 (see below) shows the comparison for the zeaxanthin concentration between the groups. The concentrations as seen in the first study are lower than the ones for lutein. But in this case, for group L3 the ANOVA run $(p>0.08)$ indicates that there is not a statistically difference from the baseline. In the case of L4 group the ANOVA indicate that there is a significant difference with the baseline values $(p<0.005)$. 


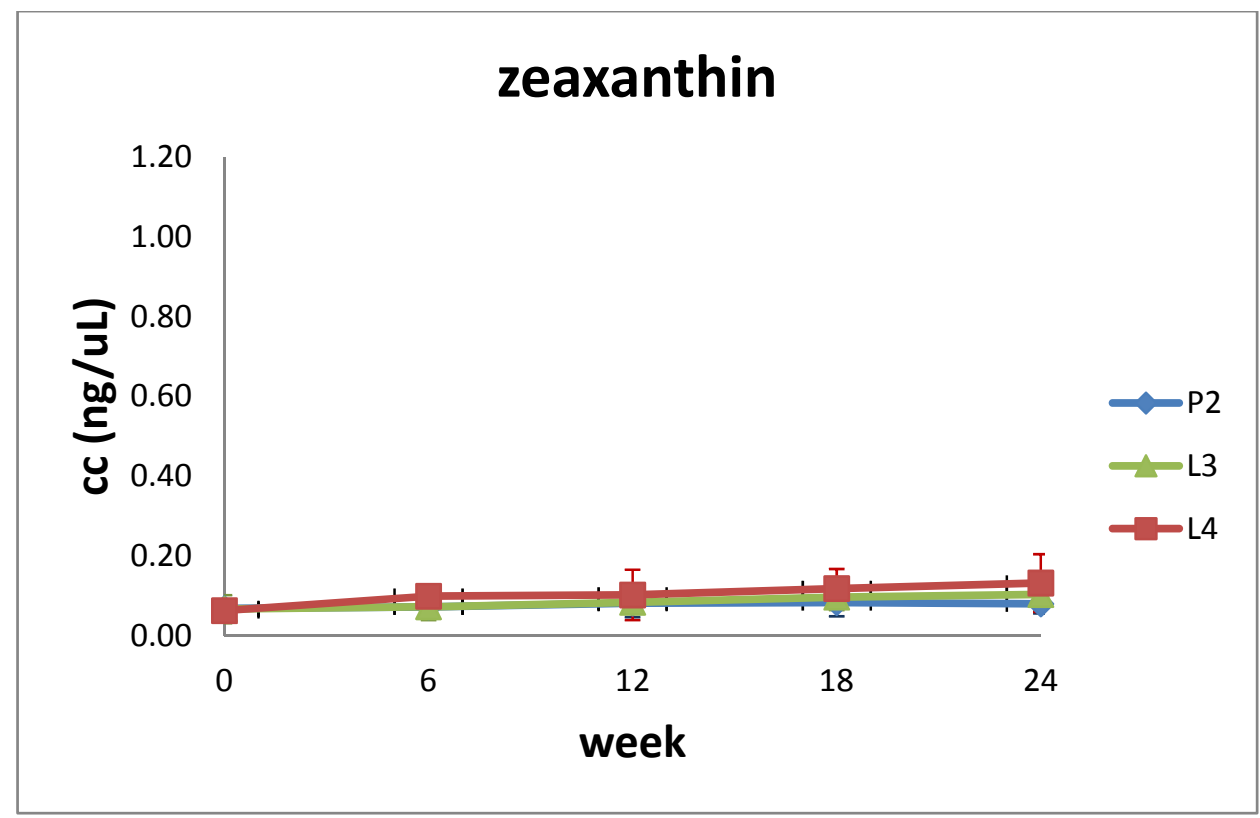

Figure 53: Comparison of zeaxanthin concentration in serum for study II.

Table 44 summarizes the average concentration for each group after supplementation (weeks 6, 12, 18 and 24) in comparison with the baseline concentrations for lutein and zeaxanthin.

Table 44: Concentrations for lutein and zeaxanthin in each group in study II.

\begin{tabular}{|cccc|ccc|}
\hline \multicolumn{3}{|c|}{ Lutein $(\mathrm{ng} / \mu \mathrm{L})$} & \multicolumn{3}{c|}{ Zeaxanthin $(\mathrm{ng} / \mu \mathrm{L})$} \\
& P2 & L3 & L4 & P2 & L3 & L4 \\
Baseline & $016 . \pm 0.05$ & $0.15 \pm 0.09$ & $0.13 \pm 0.05$ & $0.07 \pm 0.02$ & $0.07 \pm 0.04$ & $0.06 \pm 0.02$ \\
Average & $0.17 \pm 0.01$ & $0.27 \pm 0.03$ & $0.60 \pm 0.08$ & $0.08 \pm 0.00$ & $0.09 \pm 0.00$ & $0.11 \pm 0.02$ \\
\hline
\end{tabular}

In Figure 54 we can see a comparison of the average concentrations for lutein and zeaxanthin after supplementation by groups. The increase of lutein for group L3 is almost two times higher, and for group L4 is of almost 5 times higher than the corresponding to the baseline level. The increase in this case is higher for the L4 group than for the L3 group. Although the 
supplementation level was of $9 \mathrm{mg} /$ day in this case for both groups, L4 supplemented formula results to be superior to the formula use for L3. We have to highlight the fact that the formula for L4 includes other vitamins. For zeaxanthin L4 increases in almost two times the amount of the baseline. While for L3 the increase is not significant.

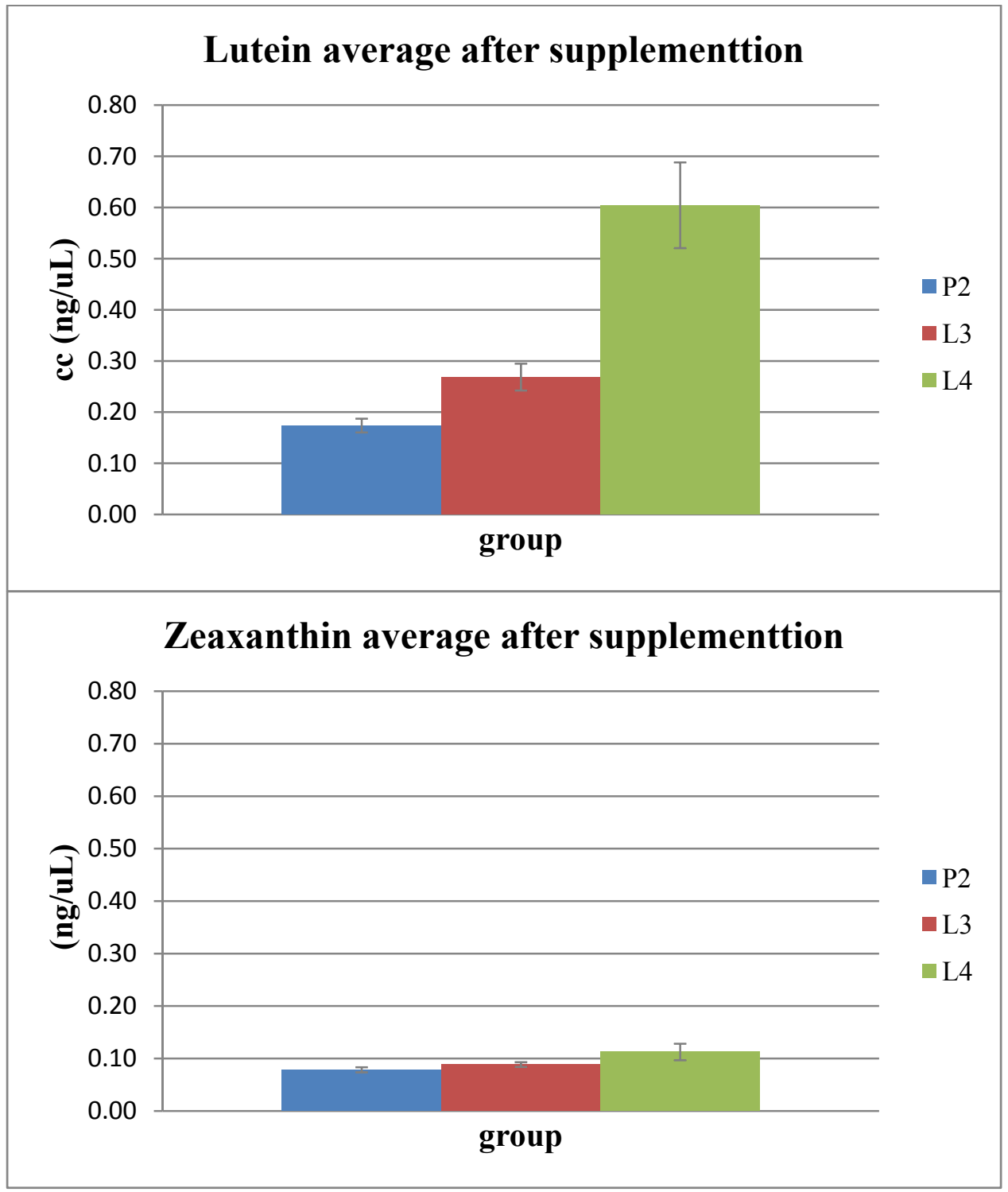

Figure 54: Average concentrations for lutein and zeaxanthin after supplementation in serum for study II. 


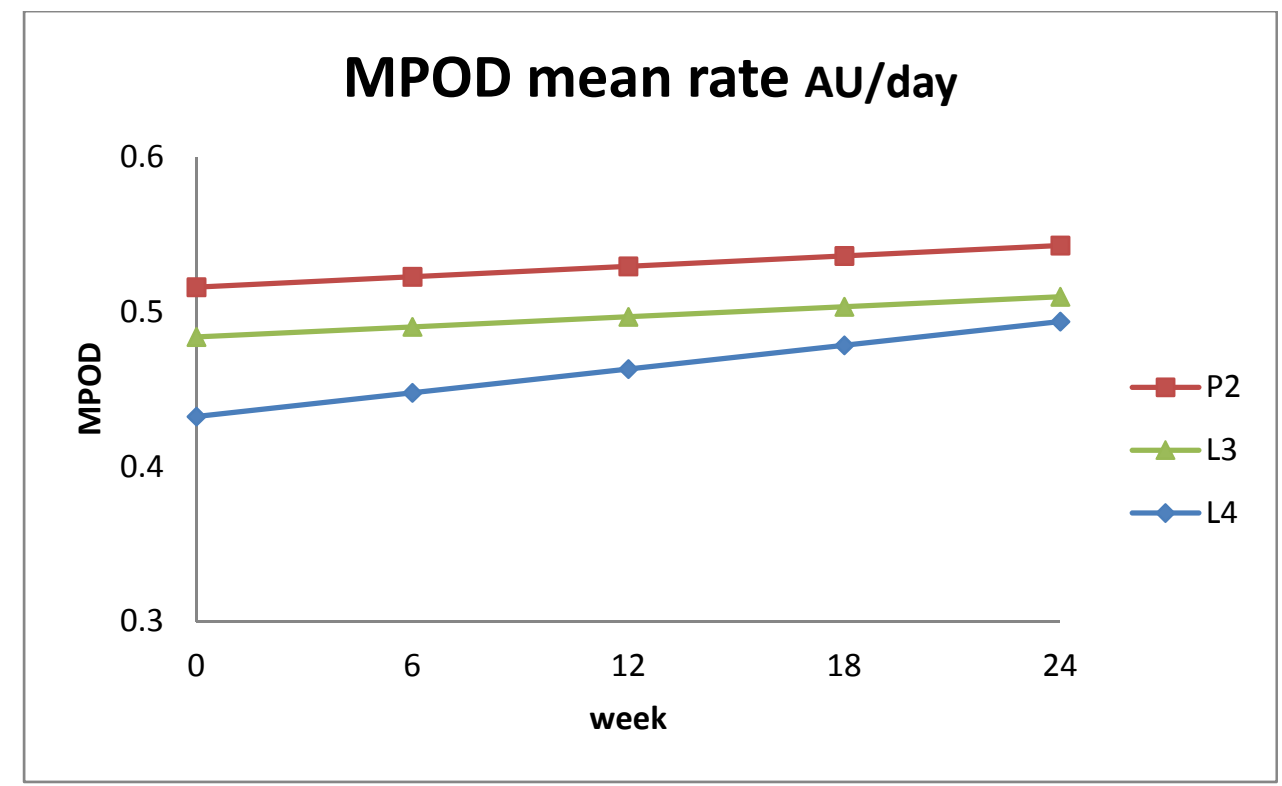

Figure 55: Comparison of mean rate of MPOD for study II.

The rate of change of MPOD was determined as the slope of a linear fit to the MPOD measures during the 24 weeks of supplementation (see table 42) and fixed to baseline of each group. The MPOD mean rate for groups P2 and L3 has similar slopes; therefore there is no difference of the means between these two groups. The variability of the MPOD mean rate for groups P2 and L3 are larger (more than 70\%) than for group L4 (23\%). There is a statistical difference between the baseline and the average of the MPOD levels for the subjects in group L4. Although the same amount of lutein was supplied to both groups: L3 and L4; there is a difference in the response.

\subsection{Conclusion}

According to the data collected in this study from 61 healthy subjects, the concentration levels of $\beta$-carotene, $\alpha$-carotene, $\beta$-cryptoxanthin, and $\alpha$-cryptoxanthin remain unaltered in human serum during the 24 weeks of study. No significant statistical difference was found in each group when the concentrations of the mentioned carotenoids that were studied were compared with the 
baseline levels in intervals of six weeks after supplementation. The absorption of the major carotenoids in the human serum was not affected by the intake of lutein supplement pills. Therefore, we can infer that the biological functions of these carotenoids are not altered, either. Lutein supplementation has no effect on these major carotenoids in the human serum, with the exception of lutein and zeaxanthin.

For the supplemented groups, the lutein concentration in human serum increased during the 24 weeks in both studies. The concentrations of lutein and zeaxanthin in the placebo groups remained at baseline levels during the study. In study I, subjects were given a $20 \mathrm{mg} /$ day dose of free lutein. Both groups showed a positive statistical difference on the average lutein concentration after supplementation, reaching up to a five-fold increase. For zeaxanthin, the average concentration was doubled. In study II, subjects were given a $9 \mathrm{mg} /$ day dose of free lutein. Only the group L4 reached similar concentration levels for lutein and zeaxanthin, as seen in study I. Although the same dose was supplied for group L3, the lutein concentration was only doubled and the zeaxanthin concentration levels did not differ from the baseline. In this case, we can conclude that the formulation used for L3 pills is not reliable. However, it should be taken into account that the formulation of L4 includes other vitamins that might help to improve the absorption of lutein in the body. Further studies have to be made to prove this idea. Although variability in the concentrations of lutein and zeaxanthin were observed when measured every six weeks, most probably due to the diet of the subjects, there was a good response when $9 \mathrm{mg}-20$ $\mathrm{mg}$ /day of lutein were supplied.

The MPOD increased in the supplemented groups L1, L2, and L4, during the study. However, differences in MPOD rates of increase between the groups did not reach a statistical significant difference. For the placebo group P1, there is no difference when compared to the baseline values, as was expected. The groups P2 and L3 had similar values of MPOD rate of 
increase, and there was no difference in the MPOD values when we compared them to their baselines, or between the groups. This fact agrees with the previous statement that L3 did not produce a significant increase in the lutein and zeaxanthin concentration levels. The increase of the MPOD response for the subjects in groups L1, L2, and L4 is slower in comparison with the increase of lutein concentration in the serum. The absorption of lutein and zeaxanthin in the bloodstream is faster than the transport of those carotenoids to the macula. The transport is associated with a selective protein-binding mechanism. This may explain the difference in the rate of increase of levels in serum and in MPOD.

Studies have showed that the consumption of lutein is low in a normal human diet. In this study, we showed that lutein supplementation does not influence the levels of the other major carotenoids in the human serum. The concentration levels of lutein and zeaxanthin increased during supplementation. The increase of these two carotenoids can lead to increased macular pigment density in the eye, which helps to protect the retina from photoxidation and lower the risk of macular degeneration. Therefore, we can suggest that lutein is a good supplementation to be included in a daily diet. 


\section{BIBLIOGRAPHY}

1. Tapiero, H., D.M. Townsend, and K.D. Tew, The role of carotenoids in the prevention of human pathologies. Biomedecine \& Pharmacotherapy, 2004. 58(2): p. 100-110.

2. Perera, C.O. and G.M. Yen, Functional Properties of Carotenoids in Human Health. International Journal of Food Properties, 2007. 10(2): p. 201-230.

3. Goodwin, T.W., The Biochemistry of the Carotenoids. Vol. 1. 1980, London: Chapman and Hall. pp. 377.

4. Cunningham, F.X. and E. Gantt, Genes and enzymes of carotenoid biosynthesis in plants. Annual Review of Plant Physiology and Plant Molecular Biology, 1998. 49: p. 557-583.

5. Hirschberg, J., Carotenoid biosynthesis in flowering plants. Current Opinion in Plant Biology, 2001. 4(3): p. 210-218.

6. Fox, D.L., Carotenoids. Otto Isler, Hugo Gutmann, and Ulrich Solms, Eds. Birkhäuser, Basel, 1971. 932 pp., illus. 118 Sw. Fr. Chemische Reihe, vol. 23. Science, 1972. 177(4043): p. 48-49.

7. Sandmann, G., Carotenoid biosynthesis in microorganisms and plants. European Journal of Biochemistry, 1994. 223(1): p. 7-24.

8. Zechmeister, L., Cis-trans isomeric carotenoids vitamins A, and arylpolyenes 1962, New York: Academic Press Inc. Publishers.

9. Gillam, A.E.E.R., M.S., The isomerization of carotenes by chromatographic adsorption: Pseudo-alpha-carotene. Biochemical Journal, 1936. 30(9): p. 1735-42.

10. Zechmeister, L. and A. Polgár, cis-trans Isomerization and Spectral Characteristics of Carotenoids and Some Related Compounds. Journal of the American Chemical Society, 1943. 65(8): p. 1522-1528.

11. Isaacson, T., et al., Cloning of tangerine from Tomato Reveals a Carotenoid Isomerase Essential for the Production of $\beta$-Carotene and Xanthophylls in Plants. The Plant Cell Online, 2002. 14(2): p. 333-342.

12. Park, H., et al., Identification of the Carotenoid Isomerase Provides Insight into Carotenoid Biosynthesis, Prolamellar Body Formation, and Photomorphogenesis. The Plant Cell Online, 2002. 14(2): p. 321-332.

13. Rao, A.V. and S. Agarwal, Role of lycopene as antioxidant carotenoid in the prevention of chronic diseases: A review. Nutrition Research, 1999. 19(2): p. 305-323.

14. Britton, G.L.-J., Synnove; Pfander, Hanspeter ed. Carotenoids: Nutrition and Health. Vol. 5. 2009, Birkhäuser Basel product: Basel. 
15. Khachik, F., et al., Effect of food preparation on qualitative and quantitative distribution of major carotenoid constituents of tomatoes and several green vegetables. Journal of Agricultural and Food Chemistry, 1992. 40(3): p. 390-398.

16. Updike, A.A. and S.J. Schwartz, Thermal Processing of Vegetables Increases Cis Isomers of Lutein and Zeaxanthin. Journal of Agricultural and Food Chemistry, 2003. 51(21): p. 6184-6190.

17. Khachik, F., et al., Isolation and structural elucidation of the geometrical isomers of lutein and zeaxanthin in extracts from human plasma. Journal of Chromatography: Biomedical Applications, 1992. 582(1-2): p. 153-166.

18. Khachik, F., et al., Identification, Quantification, and Relative Concentrations of Carotenoids and Their Metabolites in Human Milk and Serum. Analytical Chemistry, 1997. 69(10): p. 1873-1881.

19. Landrum, J.T., Bone, R.A., Chen,Y., Herrero, C., Llerena, C. M., and Twarowska, E., Carotenoids in the human retina. Pure Appl. Chem., 1999. 71(12): p. 2237-2244.

20. Bone, R.A., et al., Stereochemistry of the human macular carotenoids. Investigative Ophthalmology \& Visual Science, 1993. 34(6): p. 2033-40.

21. Landrum, J.T. and R.A. Bone, Lutein, Zeaxanthin, and the Macular Pigment. Arch. Biochem. Biophys., 2001. 385: p. 28-40.

22. Zaripheh, S. and J.W. Erdman, Factors That Influence the Bioavailablity of Xanthophylls. The Journal of Nutrition, 2002. 132(3): p. 531S-534S.

23. Shannon Carpentier, M.K., Miyoung Suh, Associations between Lutein, Zeaxanthin, and Age-Related Macular Degeneration: An Overview. Critical Reviews in Food Science and Nutrition, 2009. 49: p. 313-326.

24. Li, B., P. Vachali, and P.S. Bernstein, Human ocular carotenoid-binding proteins. Photochemical \& Photobiological Sciences, 2010. 9(11): p. 1418-1425.

25. Lee, C.M., et al., Review of Animal Models in Carotenoid Research. The Journal of Nutrition, 1999. 129(12): p. 2271-2277.

26. Li, B., et al., Identification of StARD3 as a Lutein-Binding Protein in the Macula of the Primate Retina. Biochemistry, 2011. 50(13): p. 2541-2549.

27. Beatty, S., et al., Macular pigment and age related macular degeneration. Br. J. Ophthalmol., 1999. 83: p. 867-877.

28. Landrum, J.T., et al., A One Year Study of the Macular Pigment: The Effect of 140 Days of a Lutein Supplement. Exp. Eye Res., 1997. 65: p. 57-62. 
29. Seddon, J.M., et al., Dietary Carotenoids, Vitamins A, C, and E, and Advanced AgeRelated Macular Degeneration. JAMA: The Journal of the American Medical Association, 1994. 272(18): p. 1413-1420.

30. Age-Related Eye Disease Study Research Group, The Relationship of Dietary Carotenoid and Vitamin A, E, and C Intake With Age-Related Macular Degeneration in a CaseControl Study: AREDS Report No. 22. Arch Ophthalmol, 2007. 125(9): p. 1225-1232.

31. Krinsky, N.I., Singlet oxygen in biological systems. Trends in Biochemical Sciences, 1977. 2(2): p. 35-38.

32. Krinsky, N.I., Carotenoids as chemopreventive agents. Prev Med, 1989. 18(5): p. 592602.

33. Foote, C.S., Photosensitized oxygenations and the role of singlet oxygen. Accounts of Chemical Research, 1968. 1(4): p. 104-110.

34. Mattsson Hultén, L., M. Holmström, and B. Soussi, Harmful singlet oxygen can be helpful. Free Radical Biology and Medicine, 1999. 27(11-12): p. 1203-1207.

35. McNaught, A.D., ed. Compendium of Chemical Terminology. 2nd. ed. 1997, Blackwell Science. 464.

36. De Mare, G.R. and D. Neisius, Ab initio study of rotational isomerism in 1,3-butadiene. Effect 21 of geometry optimization and basis set size on the barriers to rotation and on the stable rotamers. Journal of Molecular Structure: THEOCHEM, 1984. 109(1-2): p. 103-126.

37. Mui, P.W. and E. Grunwald, Enthalpy change for the s-trans and s-cis conformational equilibrium in 2-methyl-1,3-butadiene (isoprene), as studied by high-temperature ultraviolet absorption spectroscopy. The Journal of Physical Chemistry, 1984. 88(25): p. 6340-6344.

38. Gong, X. and H. Xiao, Ab initio study on the internal rotation of five $\pi$-conjugated hydrocarbons at MP2 level. International Journal of Quantum Chemistry, 1998. 69(5): p. 659-667.

39. Landrum, J.T., et al., The conformation of end-groups is one determinant of carotenoid topology suitable for high fidelity molecular recognition: A study of [beta]- and [epsilon]-end-groups. Archives of Biochemistry and Biophysics, 2010. 493(2): p. 169174.

40. Bassi, R., et al., Carotenoid-binding proteins of photosystem II. European Journal of Biochemistry, 1993. 212(2): p. 297-303.

41. Bhosale, P., et al., Purification and Partial Characterization of a Lutein-Binding Protein from Human Retina. Biochemistry, 2009. 48(22): p. 4798-4807. 
42. Stewart, J.J.P., Optimization of parameters for semiempirical methods I. Method. Journal of Computational Chemistry, 1989. 10(2): p. 209-220.

43. Frisc, M.J., et al., Gaussian 98, Revision A.6. 1998, Gaussian, Inc., : Pittsburgh PA.

44. Eroglu, E., et al., Comparative QSTR Study Using Semi-Empirical and First Principle Methods Based Descriptors for Acute Toxicity of Diverse Organic Compounds to the Fathead Minnow. International Journal of Molecular Sciences, 2008. 8(12): p. 12651283.

45. Foresman, J.B., Frisch, A. E., Exploring chemistry with electronic structure methods. 1996, Pittsburgh, PA: Gaussian, Inc.

46. Kavana-Saebø, K., S. Saebø, and J.E. Boggs, Geometries and rotation barriers of 2methyl-1,3-butadiene and of 2,3-dimethyl-1,3-butadiene by ab initio gradient calculation. Journal of Molecular Structure: THEOCHEM, 1984. 106(3-4): p. 259-269.

47. Khachik, F., P.S. Bernstein, and D.L. Garland, Identification of lutein and zeaxanthin oxidation products in human and monkey retinas. Invest Ophthalmol Vis Sci, 1997. 38(9): p. 1802-11.

48. Li, L., et al., Supplementation with lutein or lutein plus green tea extracts does not change oxidative stress in adequately nourished older adults. The Journal of Nutritional Biochemistry, 2010. 21(6): p. 544-549.

49. Koh, H.H., et al., Plasma and macular responses to lutein supplement in subjects with and without age-related maculopathy: a pilot study. Exp Eye Res, 2004. 79(1): p. 21-7.

50. Berson, E.L., et al., Clinical Trial of Lutein in Patients With Retinitis Pigmentosa Receiving Vitamin A. Arch Ophthalmol, 2010. 128(4): p. 403-411.

51. Nimmagadda, A.P., et al., Effect of Oral $\beta$-Carotene Supplementation on Plasma Human Immunodeficiency Virus (HIV) RNA Levels and CD4+ Cell Counts in HIV-Infected Patients. Clinical Infectious Diseases, 1998. 27(5): p. 1311-1313.

52. Fawzi, W.W., et al., A Randomized Trial of Multivitamin Supplements and HIV Disease Progression and Mortality. New England Journal of Medicine, 2004. 351(1): p. 23-32.

53. Bone, R.A., et al., Lutein and Zeaxanthin in Eyes, Serum and Diet of Human Subjects. Exp. Eye Res., 2000. 71: p. 239-245.

54. Rajendran, V., Y.S. Pu, and B.H. Chen, An improved HPLC method for determination of carotenoids in human serum. Journal of Chromatography B, 2005. 824(1-2): p. 99-106.

55. Thibeault, D., et al., Isocratic rapid liquid chromatographic method for simultaneous determination of carotenoids, retinol, and tocopherols in human serum. Journal of Chromatography B, 2009. 877(11-12): p. 1077-1083. 
56. Institute of Medicine . Panel on Dietary, A. and C. Related, Dietary reference intakes for vitamin $C$, vitamin E, selenium, and carotenoids : a report of the Panel on Dietary Antioxidants and Related Compounds, Subcommittees on Upper Reference Levels of Nutrients and of Interpretation and Use of Dietary Reference Intakes, and the Standing Committee on the Scientific Evaluation of Dietary Reference Intakes, Food and Nutrition Board, Institute of Medicine. 2000, Washington, D.C.: National Academy Press.

57. Johnston, C.S., C.A. Taylor, and J.S. Hampl, More Americans Are Eating "5 A Day" but Intakes of Dark Green and Cruciferous Vegetables Remain Low. The Journal of Nutrition, 2000. 130(12): p. 3063-3067.

58. Britton, G., S. Liaaen-Jensen, and H. Pfander, eds. Handbook of Carotenoids. 2004, Birkhauser-Verlag: Basel. 670.

59. Lee, B.B., P.R. Martin, and A. Valberg, The physiological basis of heterochromatic flicker photometry demonstrated in the ganglion cells of the macaque retina. The Journal of Physiology, 1988. 404(1): p. 323-347.

60. Bone, R. and J. Landrum, Heterochromatic flicker photometry. Arch Biochem Biophys, 2004. 430: p. 137-42. 